\title{
ÜBER DEN HABITUS DER KONFORMEN ABBILDUNG AM RANDE DES ABBILDUNGSBEREICHES.
}

\author{
VoN
}

\author{
ALEXANDER OSTROWSKI
}

in BASER.

\section{Inhaltsübersicht.}

Literaturzusammenstellung . . . . . . . . . . . . . . . . . . . . . . . . 83

Einleitung. . . . . . . . . . . . . . . . . . . . . . . . . . . . . . 85

Teil I. Der Hauptfall des Randverzerrungssatzes.

§ I. Bezeichnungen und Definitionen . . . . . . . . . . . . . . . . . . . I. Gleichmässigkeit bei $\overline{\mathrm{Lim}}$. und Lim. - 2. Konvergenz aus dem Innern des Einheitskreises. -3 . Konvergenz aus dem Innern eines allgemeinen Gebietes. - 4. L-Tangenten. - 5. Grenzstützen. - 6. Eckendefinition im Fall der allgemeinen Berandung.

$\S$ 2. Erste Ableitung der Poissondarstellung einer analytischen Funltion . . . . . 7. Verhalten in einem Stetigkeitspunkt der Belegung. (Satz I). - 8. Beweis des Satzes I. - 9. Bemerkungen zum Satz I. - Io. Die Hilfsfunktion $g_{0}(z)$. (Sätze II, III).

§ 3. Der Randverzerrungssatz . . . . . . . . . . . . . . . . . . . . . 100

I I. Formulierung des Randverzerrungssatzes. (Satz IV). - I2. Beweis nnter zwei speziellen Annahmen. - 13. Zuendeführung des Beweises von Satz IV. - I4. Die WinkeIstetigkeit der Drehung am Rande. (Satz V). - I5. Bemerkungen über die Bedeutung des Satzes V. - I6. Der Randverzerrungssatz unter der Annahme der Winkelproportionalität. $\left(\operatorname{Satz} I V^{\circ}\right)$.

$\S$ 4. Bemerkungen zum Lindelöfschen Satz . . . . . . . . . . . . . . . .

I7. Der Lindelöfsche Satz. (Satz VI). - I I. Übertragung des Lindelöfschen Satzes auf $L$-Tangentenecken. - I9. Der Lindelöfsche Satz für halbseitige Konvergenz. (Satz VII). - 20. Folgerungen aus Satz VII. (Lemmata I und 2, Zusätze I und 2 zum Satz VII).

§ 5. Relative Konformitat bei der Eckenabbildung . . . . . . . . . . . . . 116 2I. Die Längentreue im Kleinen. (Satz VIII). - 22. Beweis von $(2 \mathrm{I}, 2)$ bzw. $\left(2 \mathrm{I}, 2^{\circ}\right)$ für $\gamma=\gamma_{1}$. - 23. Der Fall $\gamma \gtrless \gamma_{1}$. -24 . Winkeltrene bei der Dreiecksabbildung.

11-34472. Acta mathematica. 64. Imprimé le 30 octobre 1934. 
(Satz IX). - 25. Beweis von Satz IX. - 26. Seitenähnlichkeit bei der Dreiecksabbildung. (Satz X). - 27. Beweis von a) für eine Dreiecksumgebung. - 28. Beweis des Satzes X. - 29. Eine Bemerkung zum Satz X.

§ 6. Vereinzelte Korollare zum Randverzerrungssatz. . . . . . . . . . . . . 30. Satz von der Winkelableitung. (Satz XI). - 3I. Versehärfung einer Ungleichung von Herrn S. WARschawski. - 32. Asymptotisches Verhalten der höheren Ableitungen von $f(z)$.

\section{Teil II. Ergänzungssätze zum Randverzerrungssatz.}

\$ 7. Erste Erweiterung des Satzes I. . . . . . . . . . . . . . . . . . 33. Formulierung des Satzes XII. (Satz XII). - 34. Beweis des Satzes XII.

§ 8. Zweite Erweiterung des Satzes I. (Durchschnittsstetigkeit) . . . . . . . . 35. Asymptotische Abschätznngen von $I_{n}(z)$. - 36. Punkte der Durehschnittsstetigkeit von $\chi(\boldsymbol{y})$. (Satz XIII). - 37. Beweis von Satz XIII. - 38. Die Schwankung von $f(z)$ (Satz XIV).

$\S$ 9. Dritte Erweiterung des Satzes I. (Durchschnittsendlichkeit) . . . . . . . .

39. A bschätzung von $f^{(n)}(z)$ für einen speziellen Fall der Durchschnittsenđlichkeit. 4o. Berückșichtigung der Gleichmässigkeit und allgemeiner Fall der Durchschnittsendlichkeit. - 4I. Einfluss der Variablentransformationen anf die Durchschnittsendlichkeit. (Satz XV, Zusatz zum Satz XV). - 42. Transformation von $z=\mathrm{I}$ auf $z=e^{i \vartheta_{0}}$. - 43. Dritte Erweiterung des Satzes I. (Satz XVI). - 44. Beweis des Satzes XVI. - 45. Zusätze zum Satz XVI. (Zusätze I, 2, 3, 4).

$\S$ го. Eine Bedingung für die Darstellbarkeit durch das Poissonsche Integral . . . 46. Formulierung der Sütze. (Sätze XVII, XVIII). - 47. Hilfssätze. (Lemmata 3, 4, 5). - 48. Beweis der Sätze XVII, XVIII. Anwendung des Spiegelungsprinzips. 49. Der Hanptteil von $f_{1}(z)$ für $z=1$.

\$ I I. Erweiterungen des Randverzerrungssatzes . . . . . . . . . . . . . . .

50. Bemerkungen über die gleichmässige Gültigkeit des Satzes IV. - 51. Der Fall einer Durchsehnittstangente. - 52. Spezialisierung des Satzes XVI für $n=1$. 53. Die verallgemeinerten Voraussetzungen. -- 54. Anwendung des Satzes XVI. 55. Der verallgemeinerte Randverzerrungssatz. (Satz XIX, Zusatz zum Satz XIX). - 56. Der Fall eines allgemeinen Gebietes $G$. - 57. Der Fall eines Gebietes $G$ mit einer Ecke in $z_{0}$ (Satz $\mathrm{XX}$ ). - 58. Gleichmässige und allseitige Stetigkeit der Drehung am Rande. - 59. Das Analogon von $(\mathrm{I} 4,3)$ unter den Voraussetzungen des Satzes XIX. - 6o. Das Analogon von $(14,1)$ unter den Voraussetzungen des Satzes XIX. - 6I. Herleitung des Lindelöfschen Satzes. -62 . Grössenordnung von $f(z)$ und $f^{\prime}(z)$ (Sätze XXI, XXI ${ }^{\circ}$.

§ I 2. Ergänzungssätze zum Randverzerrungssatz für Spitzenabbildungen . . . . . . 63. Allgemeine Charakterisierung der Resultate. - 64. Relative Sehwankung der absoluten Verzerrung am Rande (Satz XXII). - 65. Ein Gegenbeispiel. - 66. Vorbereitende Transformation von $G$. $-67 . \arg f^{\prime}(z)$ bei der Abbildung einer $L$-Tangentenspitze. - 68. Das Verzerrungsverhältnis im Fall von $L$-Tangentenspitzen (Satz XXIII): 


\section{Literaturzusammenstellung. ${ }^{1}$}

L. Ahlfons, (I) Untersuchungen zur Theorie der konformen Abbildung und der ganzen Funktionen, Acta societatis scientiarum Fennicae, Nova series A, I, Nr. 9 (1 930) pp. r -40 .

L. Bieberbach, (I) Über die Kreisabbildung von schlichten nahezu kreisförmigen Bereichen, Sitzber. der Berl. Akad., Math. phys. Klasse (1924) pp. I 8 II88. - (2) Lehrbuch der Funktionentheorie, Bd. 2, 2-te Auflage (1932).

M. Biernacki, ( $\mathrm{I}$ ) Sur l'allure de la représentation conforme dans lè voisinage d'un point exceptionel, Mathematica, Cluj, Vol. V (193 I) pp. I-6.

P. Bessonoff et M. Lavrentieff, (I) Sur l'existence de la dérivée limite, Bull. de la soc. math. de France, Vol. 58, fasc. I-II, pp. I75- 98.

H. BoHR, (r) Über streckentreue und konforme Abbildung, Math. Ztschr, Bd. r ( Igr8) pp. $403-420$.

C. Carathéodory, (I) Elementarer Beweis für den Fundamentalsatz der konformen Abbildung, Schwarz-Festschrift (I9I4), r9-41. - (2) Conformal Representation, Cambridge Tracts, (1932). - (3) Über Winkelderivierte von beschränkten analytischen Funktionen, Sitzber. der Berl. Akad., Math. phys. Klasse ( 1929 ) pp. $39-54$.

I. G. VAN DER CoRput, (I) Über die Winkelableitung bei konformer Abbildung, Akad. Wetensch. Amsterdam, Proc. 35 (r932) pp. 330-334. .

A. Hoborski, (r) Intégration de l'équation différentielle aux dérivées partielles $\frac{\partial V}{\partial t}=\frac{\partial^{2} V}{\partial x^{2}}+\frac{\partial^{2} V}{\partial y^{2}}$, Prace matematyczno-fizyczne, Tom XX, (I909) pp. I-I 43. (Polnisch).

W. GRoss, (I) Zum Verhalten analytischer Funktionen in der Umgebung singulärer Stellen, Math. Ztschr. Bd. 2 ( (918) pp. 241-294. - (2) Zum Verhalten der konformen Abbildung am Rande, Math. Ztschr. Bd. 3 (r9 19) pp. 44-64.

O. D. Kellog, (I) Harmonic functions and Greens integral, Transact. of the Americ. Math. Society I 3 (I9I2) pp. Io9-132.

E. Landau und G. Valiron, (x) A deduction from Schwarz' Lemma, Journ. of the London Math. Society 4, March (1929) pp. I62-163.

M. LavrentiefF, (I) Sur la représentation conforme, C. R. 184 (19271) pp. 1407 $-r 409$. - (2) Siehe Bessonoff-Lavrentieff ( $\mathrm{I}$ ).

L. Lichtensteln, (r) Zur konformen Abbildung einfach zusammenhängender Gebiete, Arch. der Math. u. Phys. (3) 25, (1917) pp. I79-180.-(2) Ueber das Poissonsche Integral und über die partiellen Ableitungen zweiter Ordnung des logarithmischen Potentials, Crelles J. Bd. I4I ( I9 I 2) pp. I2-42.

E. LINDELöF, (I) Sur la représentation conforme d'une aire simplement connexe sur l'aire d'un cercle, C. R. du quatrième congrès des math. scand. à Stockholm (I9 16) pp. 59-9o.

${ }^{1}$ Die in diesem Verzeichnis aufgeführten Arbeiten werden im Text nur mit der Angabe des Verfassers und der Nummer der Arbeit zitiert. 
D. Mexchoff, (I) Sur la représentation conforme des domaines plans, Math. Ann. Bd. 95 ( 1926$)$ pp. $64 \mathrm{I}-670$.

P. Montel, (1) Leçons sur les familles normales des fonctions analytiques et leurs applications, Paris 1927 .

R. Nevaninna, (I) Remarques sur le lemme de Schwarz, C. R. I88 (19291), I027 - I029. - (2) Ueber beschränkte analytische Funktionen, Commentationes in honorem Ernesti Leonardi Lindelöf, Helsinki (r929) pp. I-75.

A. Ostrowski, (I) Ueber die Bedeutung der Jensenschen Formel für die komplexe Funktionentheorie, Acta litterarum ac scientiarum, Reg. Univ. Hung. Francisco Josph. T. I, Fase. II (1923), pp. 80-87. - (2) Über quasi-analytische Funktionen und Bestimmtheit asymptotischer Entwickelungen, Acta math., Bd. 53 (1930) pp. $181-266$.

P. Painlevé, (I) Sur la théorie de la représentation conforme, C. R. I I 2 , $\left(189 x_{1}\right)$ pp. $653-657$.

R. Remak, (I) Ueber winkeltrene und streckentreue Abbildung an einem Punkte und in der Ebene, Rend. del circolo math. di Palermo, Bd. 38 (I9 I 4 ) pp. I 9 I-246.

M. Riesz, (I) Sur certaines inégalités dans la théorie des fonctions avec quelques remarques sur les géométries non-euclidiennes, Kungl. Fysiogr. Sällsk. Lund. För. I (I93I).

L. Schlesinger, (I) Automorphe Funktionen, Berlin I 924 . .

T. Shrmizu, (I) On the Domain of Indetermination of a Regular Function, Japanese Journ. of Math. Vol. VII (1930) pp. 275-300.

W. Skidel, (r) Ueber die Ränderzuordnung bei konformer Abbildung, Math. Ann. Bd. ro4 (I93 I) pp. I $83-243$.

V. Sminnoff, (I) Über die Ränderzuordnung bei konformer Abbildung, Math. Ann. Bd. $107(1932)$ pp. 313-323.

G. Valiron, (I) Siehe Landau und Valiron (I). - (2) Sur un théorème de Julia, étendant le lemme de Schwarz, Bull. des sciences math. (1929) pp. 70 -76. - (3) Sur la dérivée angulaire dans la représentation conforme, Bull. des sciences math. $5^{6}$ (1932) pp. $208-2 \mathrm{rr}$.

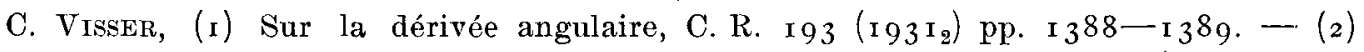
Ueber beschränkte analytische Funktionen und die Randverhältnisse bei konformer Abbildung, Math. Ztschr. Bd. 107 (1933) pp. 28-39.

S. Warschawski, (1) Ueber das Randverhalten der Ableitung der Abbildungsfunktion bei konformer Abbildung, Math. Ztschr. Bd. 35 (1932) pp. 32 I-456. - (2) Ueber einen Satz von Herrn O. D. Kellog, Nachr. d. Gesell. der Wiss. zu Göttingen, Math.-Phys. Klasse (r932) pp. 73-86.

J. WoLfF, ( $\mathrm{x}$ ) Sur la dérivée angulaire dans la représentation conforme, C. R. Igo $($ 19301) pp. 575-576. - (2) Sur la dérivée angulaire, C. R. I9I (1930 pp. $92 \mathrm{I}-923$. - (3) Sur la fonction harmonique conjuguée d'une fonction harmonique bornée, C. R. I 97 (I9332) pp. I I $80-$ II 82 .

S. Zaremba, ( $\mathrm{r}$ ) Solution générale du problème de Fourier, Acad. d. Wissenschaften, Krakau, Math.-nat. Klasse (1905) pp. 69-r68. 


\section{Einleitung.}

Man nennt eine Abbildung einer vollen Umgebung $\omega$ eines Punktes $z_{0}$ durch eine in $\omega$ stetige Funktion $w=f(z)$ konform im Punkte $z_{0}$, falls für $z \rightarrow z_{0}$ der Quotient $\frac{f(z)-f\left(z_{0}\right)}{z-z_{0}}$ einem von o verschiedenen endlichen Grenzwert zustrebt. $^{2}$ Und offenbar kann diese Definition ohne weiteres auch dann benutzt werden, wenn $\omega$ eine Teilumgebung von $z_{0}$ ist, z. B., falls $z_{0}$ ein Randpunkt eines einfach zusammenhängenden Gebietes ist, in dem $f(z)$ stetig ist. Geometrisch lässt sich der in der Forderung der Existenz eines Grenzwertes von $\frac{f(z)-f\left(z_{0}\right)}{z-z_{0}}$ steckende Sachverhalt zum Teil folgendermassen präzisieren:

Sind $z_{1}, z_{2}$ zwei in $\omega$ gelegene, gegen $z_{0}$ konvergierende Punkte, so sind zunächst die Dreiecke $\Delta_{z}=z_{0} z_{1} z_{2}, \Lambda_{w}=w_{0} w_{1} w_{2}$, wo $w_{0}, w_{1}, w_{2}$ die Bildpunkte von $z_{0}, z_{1}, z_{2}$ sind, in der Grenze ähnlich, d. h. es gilt

$$
\frac{w_{1}-w_{0}}{w_{2}-w_{0}} \sim \frac{z_{1}-z_{0}}{z_{2}-z_{0}}, \frac{w_{2}-w_{1}}{w_{2}-w_{0}} \sim \frac{z_{2}-z_{1}}{z_{2}-z_{0}}
$$

und die Differenzen entsprechender Winkel von $\Delta_{z}$ und $\Delta_{w}$ konvergieren gegen o, solange sowohl der Winkel $\alpha_{0}$ von $A_{z}$ bei $z_{0}$ als auch $\pi-\alpha_{0}$ oberhalb positiver Schranken bleiben.

Damit ist aber der Tatbestand der Konformität unserer Abbildung in $z_{0}$ nicht ausgeschöpft, da die »Grenzähnlichkeit» der Dreiecke $\boldsymbol{A}_{z}, \boldsymbol{\Delta}_{w}$ auch dann bestehen kann, wenn der Quotient $\frac{f(z)-f\left(z_{0}\right)}{z-z_{0}}$ den Grenzwert o oder $\infty$, oder überhaupt keinen Grenzwert besitzt.

Wir wollen daher unsere Abbildung von $\omega$ als relativ konform im Punkte $z_{0}$ bezeichnen, wenn die "Grenzähnlichkeit» infinitesimaler Dreiecke an $z_{0}, w_{0}$ im oben gekennzeichneten Umfang besteht. Existiert aber ein endlicher und von o verschiedener Grenzwert von $\frac{f(z)-f\left(z_{0}\right)}{z-z_{0}}$, so soll die Abbildung von $\omega$ als absolut konform in $z_{0}$ bezeichnet werden.

Sind nun $\alpha_{0}, \alpha_{1}, \alpha_{2}$ bzw. $\beta_{0}, \beta_{1}, \beta_{2}$ die Winkel der Dreiecke $\boldsymbol{\Delta}_{z}, \boldsymbol{\Delta}_{w}$ resp. bei $z_{0}, z_{1}, z_{2} ; w_{0}, w_{1}, w_{2}$, so heisst die Abbildung von $\omega$ winkeltreu in $z_{0}$, wenn

2 Die gegenseitige Abhängigkeit verschiedener Teilforderungen, die in der Forderung der Konformität stecken, untersuchen RevaK (I), Bohr (I), Menchoff (I). 


$$
\beta_{0}-\alpha_{0} \rightarrow 0
$$

gilt. Und es genügt ferner, wie man leicht einsieht, für die relative Konformität der Abbildung bereits, dass alle drei Differenzen $\beta_{0}-\alpha_{0}, \beta_{1}-\alpha_{1}, \beta_{2}-\alpha_{2}$ gegen o konvergieren und die sechs Grössen $\alpha_{0}, \alpha_{1}, \alpha_{2}, \pi-\alpha_{0}, \pi-\alpha_{1}, \pi-\alpha_{2}$ oberhalb einer positiven Schranke bleiben.

Als das wichtigste Resultat der vorliegenden Arbeit sehen wir nun die Fest. stellung an, dass, wenn bei konformer Abbildung zweier Gebiete $G_{1}, G$ in der $z$ bzw. w-Ebene aufeinander die Abbildung in zwei einander entsprechenden Randpunkten $z_{0}$ bzw. wo winkeltreu ist, sie dort auch relativ konform ist, wenn $z_{1}$ und $z_{2}$ auf eine wesentlich innerhalb $G_{1}$ liegende Winkelumgebung von $z_{0}$ beschränkt werden, d. h. auf eine solche Umgebung, die in einer gleichfalls noch innerhalb $G_{1}$ liegenden Winkelumgebung von $z_{0}$ mit gleicher Winkelhalbierenden aber grösserer Winkelöffnung enthalten ist - wir sagen in diesem Falle, dass $z_{1}$ und $z_{2}$ in $G_{1}$ gegen $z_{0}$ im Winkel konvergieren.

Ist $w=f(z)$ die unsere Abbildung vermittelnde analytische Funktion, so erweist sich die Tatsache der relativen Konformität der Abbildung als eine Folge der Tatsache, dass das Argument der Ableitung $f^{\prime}(z)$ der Abbildungsfunktion einen Grenzwert besitzt, wenn $z$ aus $G_{1}$ im Winkel gegen $z_{0}$ konvergiert; allerdings muss dabei beim Beweis der relativen Konformität mit einiger Sorgfalt verfahren werden, da ja die Seiten des Dreiecks $\Delta_{z}$ sehr wohl auch das Aussengebiet von $G_{1}$ durchsetzen können - wenn der Rand von $G_{1}$ in $z_{0}$ eine Fcke der Öffnung $>\pi$ aufweist.

Ist $G_{1}$ das Innere $K_{z}$ des Einheitskreises, so hat Hr. CARATHÉodorY ${ }^{3}$ bewiesen, dass die konforme Abbildung von $G$ auf $K_{z}$ in $z_{0}$ winkeltreu ist, wenn der Rand von $G$ im Bildpunkt $w_{0}$ von $z_{0}$ eine Tangente besitzt. Ist darüber hinaus bekannt, dass der Rand von $G$ auch in einer Umgebung von $w_{0}$ mit einer sich stetig drehenden Tangente versehen ist, so hat $\mathrm{Hr}$. E. LINDELör ${ }^{4}$ gezeigt, dass dann auch $\arg f^{\prime}(z)$ einem festen Grenzwert zustrebt, wenn $z$ aus $K_{z}$ gegen $z_{0}$ konvergiert, und $z$ darf dabei sogar allseitig aus $K_{z}$ gegen $z_{0}$ konvergieren. Andererseits hat $\mathrm{Hr}$. Lindelöf 1. c. die gleiche Tatsache auch unter einer etwas schwächeren Annahme über den Rand von $G$ in der Nähe von $w_{0}$ bewiesen, unter der Annahme nämlich, dass alle Sehnenrichtungen in der Nähe

${ }^{3}$ Carathéodory (I), pp. 38-4I; (2), pp. 9I-93; vgl. ferner Lindelöf (I), pp. 85-87, ausführlicher dargestellt nach H. FeHR und A. Puessner in Schlesinger (I), pp. 157-I6I; W. Gross (I), pp. $273-28 \mathrm{I}$.

${ }^{4}$ LindelöF (I), pp. 87-9o. 
von $w_{0}$ gegen die Tangentenrichtung in $w_{0}$ konvergieren - wir werden in diesem Falle sagen, dass unsere Kurve in $w_{0}$ eine L-Tangente besitzt.

Hr. LINDELöF hat auch aus seinem Satze gefolgert, dass unter den Voraussetzungen seines Satzes eine in $z_{0}$ mündende und sonst in $K_{z}$ verlaufende Kurve, die in $z_{0}$ mit einer stetigen Tangente mündet, in eine ebensolche Kurve der $w$-Ebene übergeht. Man kann leicht darüber hinaus beweisen, dass auch eine in $K_{z}$ verlaufende und in $z_{0}$ mit einer $L$-Tangente mündende Kurve unter den Voraussetzungen des Lindelöfschen Satzes wiederum in eine in $w_{0}$ mit einer $L$-Tangente mündende Kurve übergeht. Ferner gilt die oben festgestellte Tatsache der relativen Konformität unter den Voraussetzungen des Lindelöfschen Satzes sogar, wenn $z_{1}$ und $z_{2}$ in $K_{z}$ allseitig gegen $z_{0}$ gehen.

Und diese Tatsachen lassen sich auch auf den Fall ausdehnen, dass nur einer der in $w_{0}$ zusammenstossenden Randzweige von $G$ dort eine $L$-Tangente besitzt. In diesem Falle bleibt die relative Konformität erhalten, wenn $z_{1}$ und $z_{2}$ beliebig nahe an den entsprechenden Bogen des Einheitskreises herankommen - wir sagen dann, sie streben halbseitig an diesem Bogen gegen $z_{0}$.

Bekanntlich braucht $f(z)$ nicht einmal unter den Voraussetzungen des Lindelöfschen Satzes für $z \rightarrow z_{0}$ eine von o und $\infty$ verschiedene Ableitung zu besitzen. ${ }^{5}$ Der Frage nach der Existenz des Grenzwertes von $f^{\prime}(z)$, sowie von $\frac{f(z)-f\left(z_{0}\right)}{z-z_{0}}$ wurde in den letzten Jahren viel Aufmerksamkeit gewidmet. ${ }^{6}$ Es handelt sich in diesen Arbeiten - bis auf die Arbeiten von LavrentiefF, WarSCHAWski und Wolfe - um die Frage nach der Existenz der Winkelderivierten von $f(z)$, d. h. des Grenzwertes $\frac{f(z)-f\left(z_{0}\right)}{z-z_{0}}$ für aus $K_{z}$ gegen $z_{0}$ im Winkel strebendes $z$. Und es wurden hierfür verschiedene notwendige und hinreichende Bedingungen aufgestellt.

Für die relative Konformität der Abbildung in $z_{0}$ stellt sich aber, wie oben erwähnt, bereits die Winkeltreue in $z_{0}$ als notwendig und hinreichend heraus. Für die Winkeltreue der Abbildung ist andererseits im Falle allgemeiner Gebiete $G, G_{1}$ auf jeden Fall hinreichend, wenn $G, G_{1}$ in den betreffenden Randpunkten

5 Dies zeigt Kellog (I), pp. 122 durch Betrachtung der Abbildungsfunktion $w=z \log z$.

- Vgl. WolfFF (1), (2), (3), (4); Carathéodory (3), sowie (2), pp. 94-97; landau und VaLIRON (I), Valiron (2) und (3); LAVRENTIEFF (I) und (2); AhlFors (I); WarschaWshi (I) und (2); Seidel (I); Visser (I), (2); M. Ruesz (I); VAN DeR Corput (I); SMirnoff (I); R. NevanI.INNA (1) und (2).

Für die ersten Ansätze in dieser Richtung vgl. man PAINLEvÉ (I) sowie LiCh'TENstein (I). 
Ecken gleicher, von Null verschiedener Öffnung besitzen. Haben allgemeiner die Gebiete $G, G_{1}$ in $w_{0}$ bzw. $z_{0}$ Ecken von den Öffnungen $\gamma, \gamma_{1}$, wo $\gamma>0, \gamma_{1}>0$ ist, so ist, wie Hr. Carathéodory 1. c. bewiesen hat, die Abbildung in $z_{0}$ winkelproportional, d. h. zwei von $z_{0}$ ausgehende und ins Innere von $G_{1}$ weisende Linienelemente, die in $G_{1}$ einen Winkel $x$ miteinander bilden, gehen in zwei Linienelemente über, die in $G$ den Winkel $\frac{\gamma^{\prime}}{\gamma_{1}}$ einschliessen. In diesem allgemeineren Falle bleibt für $\gamma \neq \gamma_{1}$ die Tatsache der relativen Konformität natürlich nicht mehr richtig, es gilt aber auch in diesem Falle die Relation

$$
\frac{w_{1}-w_{0}}{w_{2}-w_{0}} \sim\left(\frac{z_{1}-z_{0}}{z_{2}-z_{0}}\right)^{\frac{\gamma}{\gamma_{1}}}
$$

wenn das Verhältnis

(c)

$$
\mid \frac{z_{2}-z_{0} \mid}{\left|z_{1}-z_{0}\right|}
$$

zwischen festen positiven Schranken bleibt. Für $\arg f^{\prime}(z)$ aber gilt im allgemeinen Falle, dass der Ausdruck

$$
\arg f^{\prime}(z)-\left(\frac{\gamma}{\gamma_{1}}-\mathrm{I}\right) \arg \left(z-z_{0}\right)
$$

einem festen Grenzwert zustrebt, wenn $z$ aus $G_{1}$ im Winkel gegen $z_{0}$ konvergiert. Aus (b) folgt ferner die Relation

$$
\frac{f^{\prime}\left(z_{2}\right)}{f^{\prime}\left(z_{1}\right)} \rightarrow \mathrm{I}
$$

Besitzen aber die vier in $z_{0}$ und $w_{0}$ zusammenstossenden Randstücke von $G_{1}$ bzw. $G$ dort $L$-Tangenten, so gilt (b) und die über (d) angegebene Tatsache auch, wenn $z_{1}, z_{2}$ bzw. $z$ aus $G_{1}$ allseitig gegen $z_{0}$ streben. Allgemeiner, ist $C_{1}$ einer der beiden in $z_{0}$ zusammenstossenden Randzweige von $G_{1}, C$ der entsprechende Randzweig von $G$ und besitzen $C_{1}$ und $C$ in $z_{0}$ bzw. $w_{0}$ L-Tangenten, so bleiben die obigen Tatsachen richtig, wenn $z_{1}, z_{2}$ bzw. $z$ halbseitig an $C_{1}$ gegen $z_{0}$ streben.

Die Existenz des Grenzwertes von (d) erschliessen wir nun aus einem Grenzwertsatz, den wir als den Randverzerrungssatz bezeichnen möchten. Er besagt im allgemeinen Falle, wenn $w=f(z) G_{1}$ auf $G$ abbildet, wo $G_{1}$ und $G$ in den 
einander entsprechenden Randpunkten $z_{0}$ bzw. $w_{0}$ Ecken von den Öffnungen $\gamma_{1}^{\prime}, \gamma$ besitzen, dass

$$
\frac{\frac{f^{\prime}(z)}{f(z)-w_{0}}}{z-z_{0}} \rightarrow \frac{\gamma}{\gamma_{1}}
$$

gilt, wenn $z$ im Winkel gegen $z_{0}$ konvergiert, wobei $\gamma_{1}>0, \gamma \geqq 0$ vorausgesetzt wird. Und diese Formel bleibt auch dann richtig, wenn über die Gebiete $G_{1}$ und $G$ nur vorausgesetzt wird, dass die konforme Abbildung von $G_{1}$ auf $G$ im Punkte $z_{0}$ winkelproportional mit dem Proportionalitätsfaktor $\frac{\gamma}{\gamma_{1}}>0$ ist. ${ }^{7}$

Der Randverzerrungssatz ergibt sich nun seinerseits sehr leicht aus einem Satz von Lichtenstein über die ersten Ableitungen des Poissonschen Integrals, wonach, wenn $P(r, \vartheta)$ der Wert eines Poissonschen Integrals im Innern des Einheitskreises $E_{z}$ ist,

$$
P_{r}^{\prime}(r, \vartheta)=o\left(\frac{\mathrm{I}}{\mathrm{I}-r}\right), \quad P_{\vartheta}^{\prime}(r, \vartheta)=o\left(\frac{\mathrm{I}}{\mathrm{I}-r}\right)
$$

gilt, wenn der Punkt $(r, 9)$ im Winkel gegen einen Stetigkeitspunkt des Integranden des Poissonschen Integrals strebt. ${ }^{8}$

Nachdem in $\$$ I der vorliegenden Abhandlung einige Bezeichnungen und Definitionen eingeführt werden, geben wir in $\$ 2$ einen neuen Beweis des Lichtensteinschen Satzes, wobei sich dieser Satz in einer insofern schärferen Fassung ergibt, als die Formeln (f) sogar für den Fall, dass $(r, 9)$ allseitig aus dem Innern von $E_{z}$ gegen einen Stetigkeitspunkt des Integranden konvergiert, nachgewiesen werden. Daraus wird nun im $\S 3$ der Randverzerrungssatz (Sätze IV, IV $^{\circ}$ ) hergeleitet, und sodann die oben über den Ausdruck (d) angegebene Tatsache gefolgert (Satz V).

Der $\$ 4$ ist dem Lindelöfschen Satz gewidmet. Es werden verschiedene Folgerungen aus diesem Satz entwickelt, namentlich auch für den Fall der halbseitigen Konvergenz. Im $\S 5$ wird für $\gamma=\gamma_{1}$ die relative Konformität der Abbildung bewiesen, und es wird für $\gamma \gtrless \gamma_{1}$ die Relation (b) hergeleitet. (Sätze VIII-X). $\S 6$ bringt endlich einige vereinzelte Folgerungen aus den bisherigen Ergebnissen;

7 In zwei später im $\$ 3$ genau angegebenen Spezialfällen findet sich (e) bei VIssER (2).

${ }^{8}$ Lichtenstein (2), pp. 20-22. Wie in Lrchtenstein (I), p. 22 angegeben wird, findet sich der Satz für eine durchweg stetige Randwertfunktion bereits bei ZAREMBA (I) sowie HoBORSKI (I). In unserer Darstellung werden allerdings die Relationen (f) und ähnliche Relationen als auf die zur Potentialfunktion $P$ gehörende analytische Funktion bezügliche Relationen formuliert.

12-34472. Acta mathematica. 64. Imprimé le 30 octobre 1934. 
so gestattet uns die Relation (b) zwei wichtige von Herrn S. WARschawski in der ersten der oben (Fussnote 6) zitierten Abhandlungen hergeleitete Ungleichungen in einer bestimmten Richtung zu verschärfen. Ebenso ergeben sich Grenzrelationen für höhere Ableitungen von $f(z)$, wenn $z$ im Winkel aus $G_{1}$ gegen $z_{0}$ konvergiert, wie z. B. die folgende $(32,3)$ :

$$
\left(z-z_{0}\right)^{v} \frac{f^{(v)}(z)}{f(z)-f\left(z_{0}\right)} \rightarrow \frac{\gamma}{\gamma_{1}}\left(\frac{\gamma}{\gamma_{1}}-\mathrm{I}\right) \cdots\left(\frac{\gamma}{\gamma_{1}}-v+\mathrm{I}\right), \nu>0 .
$$

Die im $\$ 3$ gegebene Herleitung des Randverzerrungssatzes gestattet noch nicht, den Lindelöfschen Satz mitherzuleiten. Hierzu ist es vielmehr erst nötig, den Randverzerrungssatz auf den Fall auszudehnen, dass die Ränder der abzubildenden Gebiete zwar nicht mehr notwendig Tangenten in $z_{0}$ bzw. $w_{0}$ besitzen, dass aber die Sehnenrichtungen in der Nähe von $z_{0}$ bzw. $w_{0}$ in relativ schmalen Winkelintervallen oszillieren. Die hier vorgenommene Erweiterung des Begriffes der Tangente zu demjenigen der Grenzstütze einer Kurve ist ganz analog zu der in der Theorie der reellen Funktionen vorgenommenen Erweiterung des Begriffs der Ableitung zu demjenigen einer derivierten Zahl. ${ }^{9}$ Wenn die Grenzstïtzen der Ränder von $G$ und $G_{1}$ in $w_{0}, z_{0}$ von den Schenkeln gewisser Winkel mit den Öffnungen $\gamma, \gamma_{1}$ relativ wenig abweichen, weichen auch die Unbestimmtheitsgrenzen der linken Seite von (e) nur wenig von $\frac{\gamma}{\gamma_{1}}$ ab. Aus dieser Tatsache, verbunden mit einer auf die Gleichmässigkeit der Grenzrelationen bezüglichen Ergänzung (verallgemeinerter Randverzerrungssatz, Satz XIX) lässt sich sodann der Lindelöfsche Satz unmittelbar herleiten (Nr. 6I).

Zur Herleitung des verallgemeinerten Randverzerrungssatzes ist nun eine entsprechende Frweiterung des Lichtensteinschen Satzes (Satz I) vorzunehmen. Wir nehmen sie in drei Schritten vor. Das allgemeinste Resultat wird im Satze XVI nebst Zusätzen zu diesem Satz zusammengefasst. Auch ein Teil dieses verallgemeinerten Resultats ist bereits in der oben (Fussnote 8) zitierten Lichtensteinschen Abhandlung enthalten, nämlich der Fall der Durchschnittsstetigkeit von $\chi(\vartheta)$, (Satz XIII, für $n=\mathrm{I}$ ).

Wir haben allerdings in den $\$ \$ 7-9$, in denen diese Erweiterungen des Lichtensteinschen Satzes vorgenommen werden, die Entwicklungen zum Teil weiter durchgeführt als für den unmittelbaren Zweck dieser Abhandlung notwendig

${ }^{9}$ Implizite findet sich der Begriff der Grenzstützen bereits bei W. Gross (2), pp. 276 ff. Gross spricht an der betreffenden Stelle (p. 278 unten) von 》Richtungsgrenzen der Berandung». 
gewesen wäre, um Wiederholungen bei späteren Untersuchungen zu vermeiden. Es sei hier noch auf den Satz XIV sowie den Zusatz 3 zum Satze XVI verwiesen, in denen eine, wie es scheint, bisher unbemerkt gebliebene Schwankungseigenschaft der konjugierten Funktion zum Poissonschen Potential angegeben wird. Der verallgemeinerte Randverzerrungssatz, sowie die sich daran anschliessenden Entwicklungen finden sich im § I I der Abhandlung.

Der $\S 12$ ist aber der Betrachtung des Falles $\gamma=0$ (Spitzenabbildung) gewidmet. Die vorhergehenden Entwicklungen sind nämlich nur zum Teil für $\gamma=0$ gültig, während die weiteren an den Randverzerrungssatz anschliessenden Betrachtungen nicht mehr in gleicher Weise durchgeführt werden können. Trotzdem bleiben einige Ergebnisse auch für $\gamma=0$ richtig, einige andere allerdings erst, wenn vorausgesetzt wird, dass die in der Spitze zusammenstossenden Randzweige dort L-Tangenten besitzen. Die Beweise dieser Tatsachen benutzen zum Teil das in $\S$ Io entwickelte Kriterium für die Darstellbarkeit eines Potentials durch das Poissonsche Integral, ein Kriterium, das hier noch besonders hervorgehoben sein möge, da in ihm ein neues und für manche Zwecke wichtiges Moment - Beschränktheit gewisser Flächenmittelwerte - benutzt wird.

Zum Schluss dieser Einleitung sei noch besonders auf eine kürzlich erschienene Abhandlung von Herrn C. VIsser ${ }^{10}$ hingewiesen, die einige Berührungspunkte mit der vorliegenden Abhandlung aufweist, worauf ich allerdings erst unmittelbar vor dem Abschluss des Manuskripts aufmerksam wurde. In der Abhandlung von Herrn Visser findet sich nämlich der Randverzerrungssatz für den Fall, dass $G_{1}$ mit dem Innern des Einheitskreises identisch und $\gamma=\pi$ ist, ferner für den Fall, dass $G_{1}$ wiederum mit dem Innern des Einheitskreises identisch ist und die Abbildungsfunktion $w=f(z)$ in $z_{0}$ eine endliche und von o verschiedene Winkelderivierte besitzt, so dass also die Abbildung im Punkte $z_{0}$ absolut konform bei Annäherung im Winkel ist. Aus seinem Fall des Randverzerrungssatzes hat Herr VIsser den Satz XI dieser Abhandlung für den entsprechenden Fall gefolgert. Die auf arg $f^{\prime}(z)$ bezüglichen Folgerungen und die sich daran anschliessenden Entwicklungen enthält dagegen die Vissersche Abhandlung nicht. Ferner ergibt sich aus seinen Betrachtungen die obige Relation (b), allerdings unter der recht speziellen Annahme, dass die Punkte $z_{1}$ und $z_{2}$ auf einem zu $z_{0}$ symmetrischen Orthogonalkreise zum Einheitskreis der $z$-Ebene liegen. 
Endlich hängen auch die allgemeinen analytischen Hilfssätze, die Herr Visser benutzt, naturgemäss mit unseren Entwicklungen über den Lichtensteinschen Satz zusammen. An den betreffenden Stellen des Textes wird darauf jedesmal in den Fussnoten ausdrücklich hingewiesen werden.

\section{Teil I. Der Hauptfall des Randverzerrungssatzes.}

\section{$\S$ I. Bezeichnungen und Definitionen.}

I. Gleichmässigkeit bei $\overline{\text { Lim }}$ und Lim. Wir werden im Folgenden vom Begriff der Gleichmässigkeit nicht nur bei der Konvergenz Gebrauch machen, sondern auch vom analogen Begriff bei Gleichheits- und Ungleichheitsbeziehungen, in denen neben den Grenzwerten auch $\overline{\mathrm{Lim}}$ und Lim vorkommen. Wenn wir z. B. von der Relation

$$
\operatorname{Lim}_{n \rightarrow \infty} f_{n}(\vartheta) \leqq \varphi(\vartheta)
$$

sagen, dass sie auf einer $\vartheta$-Menge $M$ gleichmässig gilt, so bedeutet dies, dass zu jedem $\varepsilon>0$ ein solches $N(\varepsilon)>0$ angegeben werden kann, dass für alle $n>N$ und für alle $\vartheta$ auf $M$

gilt.

$$
f_{n}(\vartheta) \leqq \varphi(\vartheta)+\varepsilon
$$

2. Konvergenz aus dem Innern des Einheitskreises. Unter $E$ werden wir in allen weiteren Ausführungen die Peripherie des Einheitskreises $|z|=\mathrm{I}$ ver. stehen; handelt es sich dabei um den Einheitskreis in der $z$-bzw. $w$-Ebene u.s.w., so werden wir, wenn es sich aus dem Zusammenhang nicht unmittelbar ergibt, auf welche Ebene $E$ zu beziehen ist, die Bezeichnung $E_{z}$, bzw. $E_{w}$ u. s. w. benutzen. Ebenso verstehen wir unter $K$ das Innere des Einheitskreises und schreiben dafür, je nach der Ebene, in der $K$ zu betrachten ist, $K_{z}, K_{w}$ u. s. w.

Ist die Variable $z$ auf das Innere von $E_{z}$ beschränkt und ist $e^{i \vartheta_{0}}$ ein Punkt von $E_{z}$, so sagen wir, $z$ konvergiere $\gg$ im Winkel» gegen $e^{i \vartheta_{0}}$, wenn $z$ gegen $e^{i \vartheta_{0}}$ strebt, dabei aber zwischen zwei vom Punkte $e^{i 9_{0}}$ ausgehenden Sehnen von $E_{z}$ bleibt. Wird aber das gegen $e^{i \varphi_{0}}$ aus dem Innern von $E_{z}$ konvergierende $z$ keinerlei derartigen Bedingungen unterworfen, so sagen wir, $z$ konvergiere »allseitig» gregen $e^{i \vartheta_{0}}$.

Endlich werden wir sagen, $z$ konvergiere gegen $e^{i \vartheta_{0}}$ "halbseitig», genauer halbseitig an einem in $e^{i \varphi_{0}}$ endenden Kreisbogen $C$ von $E_{z}$, wenn $z$ dabei zwischen 
dem Bogen $C$ und einer von $e^{i 9_{0}}$ ausgehenden Sehne von $E_{z}$ bleibt. Analoges gilt für allgemeinere Gebiete.

3. Konvergenz aus dem Innern eines allgemeinen Gebietes. Es bestehe der Rand eines einfach zusammenhängenden Gebietes $G$ in der Umgebung eines Randpunktes $P$ aus einem freien Jordanbogen. Es seien $S_{1}, S_{2}$ zwei von $P$ ausgehende geradlinige Strecken, die bis auf $P$ in $G$ liegen. Es sei $A$ ein Dreieck mit der Spitze in $P$, das, bis auf den Punkt $P$, in $G$ und zwar im Winkel zwischen den Strecken $S_{1}, S_{2}$ liegt, so dass es mit diesen Strecken nur den Punkt $P$ gemeinsam hat. Ein solches Dreieck bezeichnen wir als eine Dreiecksumgebung von $P$ innerhalb $G$. Fine Vereinigungsmenge von endlich vielen Dreiecksumgebungen von $P$ innerhalb $G$ bezeichnen wir als eine Winkelumgebung von $P$ innerhalb $G$. Strebt ein Punkt $z$ so gegen $P$, dass es dabei in einer Winkelbzw. Dreiecksumgebung von $P$ innerhalb $G$ bleibt, so sagen wir, $z$ strebe aus dem Innern von $G$ im Winkel bzw. im Dreieck gegen $P$. Ist aber $z \rightarrow P$ und keinerlei solchen Bedingungen unterworfen, bis auf die Forderung, dass $z$ in $G$ bleibt, so sagen wir, $z$ konvergiere aus dem Innern von $G$ allseitig gegen $P$.

Es sei endlich $C$ ein in $P$ mündender Randbogen von $G$. Es seien $R_{1}, R_{2}$ zwei von $P$ ausgehende und bis auf $P$ innerhalb $G$ liegende Strecken. Es möge $R_{1}$ zwischen $R_{2}$ und $C$ liegen. Man verbinde einen inneren Punkt von $R_{1}$ mit einem von $P$ verschiedenen Punkt von $C$ durch einen Jordanbogen, der bis auf seinen Schnittpunkt mit $C$ ganz in $G$ liegt. Durch diesen Jordanbogen wird ein Teilgebiet von $G$ abgegrenzt, das zwischen $R_{1}$ und $C$ liegt und $P$ als Randpunkt besitzt. Ein solches Teilgebiet bezeichnen wir als eine an $C$ liegende halbseitige Umgebung von $P$ innerhalb $G$. Konvergiert nun ein Punkt $z$ gegen $P$ und bleibt er dabei in einer solchen halbseitigen Umgebung von $P$, so werden wir sagen, er konvergiere aus dem Innern von $G$ halbseitig am Randbogen $C$ gegen $P$.

4. L-Tangenten. Es sei $\Gamma(z=z(t), \mathrm{O} \leqq t \leqq \mathrm{I})$ ein Jordanbogen. Wir sagen, er besitze im Punkte $t=0$ eine Tangente (eigentlich Halbtangente) $\tau$, wenn jedem noch so schmalen Winkel um den Halbstrahl $\tau$ mit der Spitze in $z(0)$ ein solches $\delta>0$ zugeordnet werden kann, dass alle Punkte $z(t)$ mit $0<t<\delta$ in diesem Winkel liegen. Lässt sich jedem positiven $\varepsilon$ ein positives $\delta=\delta(\varepsilon)$ derart zuordnen, dass die Richtungen sämtlicher Sehnen des Teilbogens $o \leqq t \leqq \delta$ von $I$ mit der Richtung $\tau$ einen Winkel $\leqq \varepsilon$ einschliessen, so werden wir sagen, $\boldsymbol{\Gamma}$ besitze im Punkte $t=0$ eine $L$-Tangente. ${ }^{11}$

It Der Begriff ohne Benennung kommt anscheinend zuerst bei LindeLöF (1), p. 87 unten vor. CakAthéodory (2), p. 94 sagt, die Kurve sei an den betreffenden stelle glatt (smooth). 
Wird ein Koordinatensystem mit dem Ursprung in $z(\mathrm{o})$ gelegt, in dem der Richtungstangens $\theta_{0}$ ron $\tau$ endlich ist und das in $z(0)$ einmündende Stück von $\Gamma$ "zuletzt» etwa in der rechten Halbebene liegt, so besitzt $\Gamma$ in der Umgebung von $z(\mathrm{o})$ die Gleichung $y=\varphi(x)$, und unsere Bedingung für die $L$-Tangente läuft dann auf die Bedingung hinaus, dass

$$
\frac{\varphi\left(x_{1}\right)-\varphi\left(x_{2}\right)}{x_{1}-x_{2}}
$$

gegen $\theta_{0}$ konvergiert, wenn $x_{1}$ und $x_{2}$ unabhängig voneinander, aber ohne zusammenzufallen, gegen $\circ$ abnehmen.

Man kann diese Bedingung noch anders fassen, wenn man den in der Theorie der reellen Funktionen gebräuchlichen Begriff der derivierten Zahlen benutzt. Nach einem Satz von Du BoIs-REymond fällt nämlich das Wertintervall des Differenzenquotienten $(4, \mathrm{I})$ für $a \leqq x_{1}<x_{2} \leqq b$ mit dem kleinsten Intervall zusammen, das sämtliche Werte irgendeiner der vier derivierten Zahlen von $\varphi(x)$ für $a \leqq x \leqq b$ enthält. Daher läuft unsere Definition für die L-Tangente darauf hinaus, dass irgend eine (und damit jede) der vier derivierten Zahlen von $\varphi(x)$ für $x \downarrow$ o gegen $\theta_{0}$ strebt.

5. Grenzstützen. Um unabhängig von der Orientierung des Koordinatensystems die entsprechende Bedingung formulieren zu können, führen wir den Begriff der Grenzstïtzen ein. Es sei $\Gamma(z=z(t))$ ein Jordanbogen, und man betrachte für $t>t_{0}$ die von $z\left(t_{0}\right)$ nach $z(t)$ gezogenen gerichteten Sehnen, deren analytische Richtungen ${ }^{12}$ sich für $t>t_{0}$ stetig mit $t$ ändern und nach Festlegung einer von diesen Richtungen für jedes $t>t_{0}$ eindeutig bestimmt sind. Wird die analytische Richtung der Sehne von $z\left(t_{0}\right)$ nach $z(t)$ mit $\theta(t)$ bezeichnet, und ist

$$
\theta^{+}=\overline{\operatorname{Lim}_{t \downarrow t_{0}}} \theta(t), \quad \theta^{-}=\frac{\operatorname{Lim}}{t \downarrow t_{0}} \theta(t)
$$

so nennen wir, falls $\theta^{+}, \theta^{-}$endlich sind, die von $z\left(t_{0}\right)$ ausgehenden Halbstrahlen mit den analytischen Richtungen $\theta^{+}, \theta^{-}$die rechtsseitigen Grenzstützen des entsprechenden in $z\left(t_{0}\right)$ endenden Bogens und den Winkel zwischen den analytischen

12 Unter der analytischen Richtung eines Vektors verstehen wir seinen Winkel mit einer festen Richtung, wobei aber $z$ wei $\mathrm{um}$ ein von 0 verschiedenes Vielfaches von $2 \pi$ verschiedene Winkel verschiedene analytische Richtungen festsetzen - die Richtungen werden also eigentlich auf der Riemannschen Fläche des Logaritmus aufgetragen. 
Über den Habitus der konformen Abbildung am Rande des Abbildungsbereiches. 95

Richtungen $\theta^{+}, \theta^{-}$den (rechtsseitigen) Stïtzenschwankungswinkel in $z\left(t_{0}\right)$. Er ist. also gleich $\theta^{+}-\theta^{-}$.

Offenbar kann man $\theta^{+}, \theta^{-}$auch anders definieren. Man denke sich zwei Kurvenbögen $C^{+}, C^{-}$, die in $z\left(t_{0}\right)$ einmünden und dort bestimmte Tangenten besitzen. Haben diese Tangenten die analytischen Richtungen $\theta^{+}+\varepsilon, \theta^{-}-\varepsilon_{1}$, mit $\varepsilon>0, \varepsilon_{1}>0$, so liegt $\gg$ zwischen» $C^{+}, C^{-}$das gesamte Stück von $\Gamma$ mit $t_{0}<t<t_{0}+\delta$ für hinreichend kleine $\delta$. Sind aber die Tangentenrichtungen von $C^{+}, C^{-}$etwa $\theta^{+}-\varepsilon, \theta^{-}+\varepsilon_{1}$, mit $\varepsilon>0, \varepsilon_{1}>0$, so gibt es für jedes noch so kleine positive $\delta$ Punkte $z(t)$ mit $t_{0}<t<t_{0}+\delta$, die nicht »zwischen» $C^{+}, C^{-}$ liegen. Und es ist unmittelbar klar, wie die entsprechenden Formulierungen lauten, wenn $\theta^{+}=+\infty$ oder $\theta^{-}=-\infty$ ist. Ganz analog werden die links. seitigen Grenzstützen und der linksseitige Stützenschwankungswinkel in $z\left(t_{0}\right)$ mit Hilfe des zweiten in $z\left(t_{0}\right)$ mündenden Bogens definiert.

Aus dem Gesagten geht aber nunmehr unmittelbar hervor, dass bei einer konformen Abbildung einer Umgebung von $z\left(t_{0}\right)$ auch die Grenzstützen von $\Gamma$ um den gleichen Winkel gedreht werden, wie die direkt vorgegebenen Linienelemente, und daher insbesondere die beiderseitigen Stützenschwankungswinkel un verändert bleiben. Und das Gleiche gilt natürlich auch dann, wenn nur eine Teilumgebung von $z\left(t_{0}\right)$ winkeltreu in $z\left(t_{0}\right)$ abgebildet wird, wie dies z. B. bei konformer Abbildung in der Nähe des Randes der Fall sein kann, sofern die beiden Grenzstïtzen des betrachteten Teilbogens von $\Gamma$ in der entsprechenden Winkelumgebung liegen. Endlich ist es klar, wie diese Formulierungen abzuändern sind, wenn die in Frage kommenden Abbildungen nicht mehr winkeltreu, sondern nur winkelproportional sind. Auch dann werden die Richtungen der Grenzstützen in gleicher Weise transformiert, wie die Richtungen gewöhnlicher Tangenten, und die Stützenschwankungswinkel mit dem gleichen Proportionalitätsfaktor multipliziert, wie die Winkel zwischen gewöhnlichen Linienelementen.

6. Eckendefinition im Fall der allgemeinen Berandung. Man pflegt bei der Betrachtung der Tangente oder Ecke in einem Randpunkt eines einfach zusammenhängenden Gebietes $G$ in der Regel stillschweigend vorauszusetzen, dass der Rand des Gebietes in der Umgebung des betreffenden Randpunktes einen Jordanbogen bildet. Man kann nun mit Hilfe der Theorie der Randelemente (der Carathéodoryschen Primenden) eine Parameterdarstellung für den Rand von $G$ erhalten, an Hand deren in vielen Fällen sowohl der Begriff der Tangente und 
Ecke als auch der Begriff der Grenzstützen verallgemeinert werden kann. ${ }^{14}$ Es mag indessen für das Folgende genügen, wenn wir eine auf einen hinreichend allgemeinen Fall anwendbare Definition einer Ecke geben.

Wir werden sagen, der Rand von $G$ bilde in einem Randpunkt $P$ eine Ecke, wenn es zwei von $P$ ausgehende Halbstrahlen $\tau_{1}^{*}, \tau_{2}$ gibt, derart, dass wenn man beliebig schmale Winkel um diese Halbstrahlen bildet, alle Randpunkte von $G$, die hinreichend nahe bei $P$ liegen, sich auf diese beiden Winkel verteilen. Die Halbstrahlen $\tau_{1}, \tau_{2}$ werden als die beiden Halbtangenten an den Rand ron $G$ im Punkte $P$ bezeichnet. Hat einer der beiden Winkel, die durch $\tau_{1}$ und $\tau_{2}$ gebildet werden, die Öffnung $\gamma \pi$ und die Eigenschaft, dass es in ihm Kreissektoren mit der Spitze in $P$ gibt, deren Winkel bei $P$ beliebig nahe an den Winkel zwischen $\tau_{1}$ und $\boldsymbol{\tau}_{2}$ herankommen und deren Inneres ganz in $G$ liegt, so werden wir sagen, dass der Rand von $G$ von der betreffenden Seite eine Ecke von der inneren Öffnung $\gamma \pi$ besitzt. Es kann natürlich auch der andere Winkel $2 \pi-\gamma \pi$ zugleich ein innerer Winkel $\gg$ von der anderen Seite» sein, wenn der Punkt $P$ ein Doppelpunkt des Randes ist. $\gamma$ könnte allerdings auch den Wert o haben; in diesem Falle ist der Punkt $P$ von der betreffenden Seite längs eines Jordanbogens $L$ erreichbar, es gibt aber kein Dreieck mit der Spitze in $P$, dessen Inneres ganz in $G$ liegt und zugleich beliebig an $P$ benachbarte Punkte von $L$ enthält.

\section{$\S$ 2. Erste Ableitung der Poissondarstellung einer analytischen Funktion.}

7. Verhalten in einem Stetigkeitspunkt der Randfunktion. Satz I. Es sei $\chi(\vartheta)$ eine im Intervall $(-\pi, \pi)$ beschränkte messbare Funktion, die für $\vartheta=\vartheta_{0}$ stetig ist. Denkt man sich $\chi(\vartheta)$ durch die Forderung der Periodizität mit der Periode $2 \pi$ fiir alle Werte von $\vartheta$ fortgesetzt, so möge

$$
\left|\chi(\vartheta)-\chi\left(\vartheta_{0}\right)\right|<\lambda\left(\vartheta-\vartheta_{0}\right)
$$

sein, wo die gerade Funktion $\lambda(x)$ zwischen o und $\pi$ monoton ist, fii $x \downarrow \circ \lambda(x) \downarrow \circ$ gilt, $\lambda(\pi)=C$ und für $x>\pi \lambda(x)=C$ ist. - Offenbar kann für unser $\chi(\vartheta)$ stets cine solche Funktion $\lambda(x)$ gefunden und insbesondere so gewählt werden, dass $C$ gleich der oberen Grenze von $\left|\chi(\vartheta)-\chi\left(\vartheta_{0}\right)\right|$ ist. - Bildet man mit Hilfe des Poissonschen Integrals eine zu $\chi(9)$ gehörende analytische Funktion

14 Vgl. hierzu W. Gross (1), pp. 276, 277. 


$$
f(z)=\frac{\mathrm{I}}{2 \pi} \int_{-\pi}^{\pi} \frac{e^{i \vartheta}+z}{e^{i \vartheta}-z} \chi(\vartheta) d \vartheta+i c
$$

wo c eine reelle Konstante ist, so gilt für die Ableitung

$$
f^{\prime}(z)=\frac{\mathrm{I}}{\pi} \int_{-\pi}^{\pi} \frac{e^{i \vartheta}}{\left(e^{i \vartheta}-z\right)^{2}} \chi(\vartheta) d \vartheta
$$

von $f(z)$, wenn $z$ allseitig aus $K_{z}$ gegen $e^{i \vartheta_{n}}$ strebt:

$(7,4)$

$$
(\mathrm{I}-|z|) f^{\prime}(z) \rightarrow \mathrm{o},
$$

und darïber hinaus, $z=r e^{i\left(\varphi+\vartheta_{0}\right)}, \pi \geqq \varphi \geqq-\pi$ gesetzt,

$$
(\mathrm{I}-r)\left|f^{\prime}(z)\right|<\lambda((\mathrm{I}-r) k+|\varphi|)+3 \frac{C}{k}, \mathrm{I}>r>\frac{\pi}{6},
$$

wo für $k$ eine beliebige Zahl > I eingesetzt werden kann.

8. Beweis. Ohne Beschränkung der Allgemeinheit darf $\vartheta_{0}=0$ vorausgesetzt werden. Aus $(7,3)$ folgt, da man $\chi(\vartheta)$ durch $\chi(\vartheta)-\chi\left(\vartheta_{0}\right)$ ersetzen kann,

$$
\left|f^{\prime}(z)\right| \leqq \frac{\mathrm{I}}{\pi} \int_{-\pi}^{\pi} \frac{\lambda(\vartheta) d \vartheta}{\left|z-e^{i \vartheta}\right|^{2}}=\frac{\mathrm{I}}{\pi} \int_{-\pi}^{\pi} \frac{\lambda(\vartheta+\varphi) d \vartheta}{r^{2}-2 r \cos \vartheta+\mathrm{I}}
$$

Hier ist der Nenner gleich

$$
(\mathrm{I}-r)^{2}+4 r \sin ^{2} \frac{9}{2} \geqq(1-r)^{2}+\frac{4}{\pi^{2}} r \vartheta^{2}
$$

so dass nach der Einführung der neuen Integrationsvariabeln $x=\frac{2 \vartheta \sqrt{r}}{\pi(\mathrm{I}-r)}$ aus $(8, \mathrm{I})$

$$
\left|f^{\prime}(z)\right|<\frac{\mathrm{I}}{2(\mathrm{I}-r) \sqrt{r}} \int_{-\infty}^{\infty} \frac{\lambda\left(\frac{\pi x(\mathrm{I}-r)}{2 \sqrt{r}}+\varphi\right)}{\mathrm{I}+x^{2}} d x
$$

folgt. Setzen wir zur Abkürzung $\mathrm{I}-r=\varrho$ und beachten, dass $\lambda(x)$ gerade und monoton ist, so folgt weiter

13-34472. Acta mathematica. 64. Imprimé le 30 octobre 1934. 


$$
\sqrt{r} \varrho\left|f^{\prime}(z)\right| \leqq \int_{0}^{\infty} \frac{\lambda\left(\frac{\pi \varrho x}{2 \sqrt{r}}+|\varphi|\right)}{1+x^{2}} d x=\int_{0}^{k}+\int_{K}^{\infty},
$$

wo $K$ vorläufig beliebig, aber $>$ I sei. Hier gilt aber, wegen der Monotonie von $\lambda(x)$ :

$$
\begin{aligned}
& \int_{0}^{K} \leqq \lambda\left(\frac{\pi \varrho K}{2 \sqrt{r}}+|\varphi|\right) \operatorname{arctg} K, \\
& \int_{K}^{\infty} \leqq C\left(\frac{\pi}{2}-\operatorname{arctg} K\right)=C \operatorname{arctg} \frac{\mathrm{I}}{K} .
\end{aligned}
$$

Nun gilt für $0<x<\mathrm{I}: \operatorname{arctg} x<x$. Daher ergibt sich schliesslich für $\varrho\left|f^{\prime}(z)\right|$ aus $(8,2)$ die Abschätzung durch

$$
\frac{\mathrm{I}}{\sqrt{r}} \frac{\pi}{2} \lambda\left(\frac{\pi \varrho K}{2 \sqrt{r}}+|\varphi|\right)+\frac{C}{\sqrt{r} K},
$$

oder, wenn $r>\frac{\pi}{6}$ vorausgesetzt und $K=\frac{2 \sqrt{r} k}{\pi}, k>$ I, gesetzt wird, $(7,5)$, w. z. b. w.

9. Bemerkungen zum Satz I. Je nach dem Typus der Funktion $\lambda(x)$ kann $\operatorname{man} k$ in $(7,5)$ verschieden spezialisieren. Ist z. B. $\lambda(x) \leqq C|x|^{\alpha}, 0<\alpha<\mathrm{I}$, so setze man $k=\varrho^{-\frac{\alpha}{1+\alpha}}$ und es ergibt sich

$$
\varrho\left|f^{\prime}(z)\right| \leqq \lambda\left(\varrho^{\frac{1}{1+\alpha}}+|\varphi|\right)+3 C \varrho^{\frac{\alpha}{1+\alpha}}, \varrho \leqq \mathrm{I}-\frac{\pi}{6} .
$$

Der Satz I liefert eine Abschätzung von $f^{\prime}(z)$, sobald $f(z)$ sich in der Form $(7,2)$ darstellen lässt. Dies ist z. B. bekanntlich immer der Fall, wenn $\Re f(z)$ in $K_{z}$ absolut beschränkt ist. Ist von einer Funktion $f(z)$ bekannt, dass sie in $K_{z}$ regulär und dass ihr Realteil auf $K_{z}+E_{z}$ stetig ist, so folgt daraus erst recht, wenn $\Re f\left(e^{i \vartheta}\right)=\chi(\vartheta)$ gesetzt wird, dass

$$
f(z)=\frac{\mathrm{I}}{2 \pi} \int_{-\pi}^{\pi} \frac{e^{i \vartheta}+z}{e^{i \vartheta}-z} \chi(\vartheta) d \vartheta+i c
$$


Über den Habitus der konformen Abbildung am Rande des Abbildungsbereiches. 99 gilt, wo $c$ eine reelle Konstante ist. Daher gilt für jede solche Funktion die Formel $(7,4)$ für allseitig aus $K_{z}$ gegen $E_{z}$ konvergierendes $z$.

Io. Die Hilfsfuntion $g_{0}(z)$. Satz II. Wird unter $g_{0}(z)=\lg \frac{\mathrm{I}+z}{\mathrm{I}-z}$ für. $|z|<$ I diejenige Bestimmung des Logarithmus verstanden, die für $z=0$ verschwindet, so gilt

$$
\left|\Im g_{0}(z)\right|<\frac{\pi}{2} \quad \text { für }|z|<\mathrm{I},
$$

$(10,2)$

$$
\Im g_{0}(z)-\psi \rightarrow \text { o für }|z|<\mathrm{I}, z \rightarrow \mathrm{I},
$$

$($ I 0,3$)$

$$
\mathfrak{J} g_{0}\left(e^{i \vartheta}\right)=\left\{\begin{array}{cl}
\frac{\pi}{2}, & 0<\vartheta<\pi, \\
-\frac{\pi}{2}, & 0>\vartheta>-\pi,
\end{array}\right.
$$

wo $\psi=-\arg (\mathrm{I}-z)$ ist mit $-\frac{\pi}{2}<\psi<\frac{\pi}{2}$. Ferner ist $\frac{\mathrm{I}}{i} g_{0}(z)$ durch das Poissonsche Integral $(7,2)$ mit $\mathfrak{s} g_{0}\left(e^{i \vartheta}\right)$ als Belegungsfunktion $\chi(\vartheta)$ darstellbar. Endlich gilt:

$(\mathrm{IO}, 4)$

$$
\frac{g_{0}(z)}{-\lg |\mathrm{I}-z|} \rightarrow \mathrm{I} ; \quad(\mathrm{I}-z)^{n} g_{0}^{(n)}(z) \rightarrow(n-\mathrm{I}) !
$$

wenn $z$ aus dem Innern von $E_{z}$ allseitig gegen $z=\mathrm{I}$ konvergiert.

(IO, I), (IO, 3) ergeben sich daraus, dass $w=\frac{\mathrm{I}+z}{\mathrm{I}-z}$ das Innere von $E_{z}$ auf die Halbebene $\mathfrak{R} w>0$ so abbildet, dass dabei $z=-\mathrm{I}$ in $w=\mathrm{o}$ übergeht. $(\mathrm{IO}, 2)$ folgt aus

$$
\arg \frac{\mathrm{I}+z}{\mathrm{I}-z}=\arg (\mathrm{I}+z)+\psi \text { wegen } \arg (\mathrm{I}+z)=o(\mathrm{I})
$$

für $z \rightarrow$ I. (IO, 4) endlich ergibt sich direkt aus

$$
g_{0}^{(n)}(z)=\frac{(n-\mathrm{I}) !}{(\mathrm{I}-z)^{n}}+(-\mathrm{I})^{n-1} \frac{(n-\mathrm{I}) !}{(\mathrm{I}+z)^{n}}
$$

Satz III. Unter Beibehaltung der Bezeichnungen von Satz II gilt, $z=r e^{i 9}$ gesetzt, für $\mathrm{o} \leqq r<\mathrm{I}$ und $\vartheta \rightarrow \mathrm{O}$

$$
\frac{\mathrm{I}-|z|}{|\mathrm{I}-z|}=\cos \psi+O(\vartheta)
$$


$(\mathrm{I} 0,6)$

$$
\mathrm{I}-|z|=(\mathrm{I}-z) e^{i \psi} \cos \psi+O(\vartheta) .
$$

Denn wendet man den Sinussatz auf das Dreieck $z e^{i 9} \mathbf{I}$ der Fig. I an, so ergibt sich

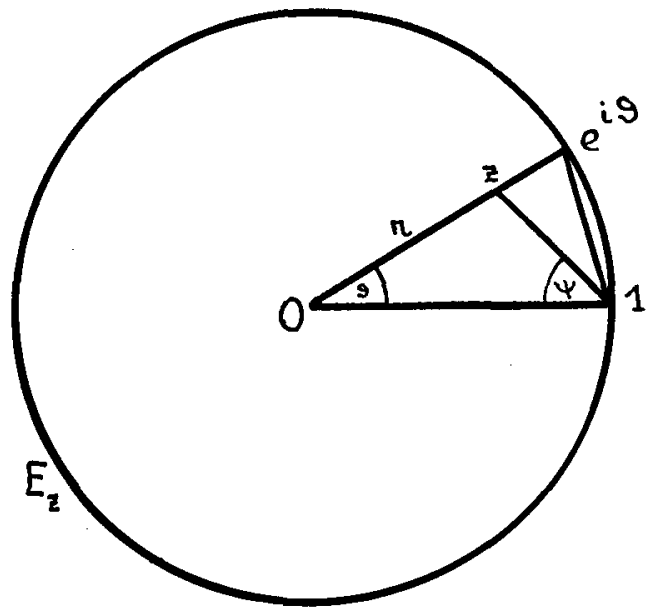

Fig. I.

$(\mathrm{ro}, 7)$

$$
\frac{2 \sin \frac{\vartheta}{2}}{\mathrm{I}-r}=\frac{\sin (\vartheta+\psi)}{\cos \left(\frac{\vartheta}{2}+\psi\right)}, \frac{1-r}{|\mathrm{I}-z|}=\frac{\cos \left(\frac{\vartheta}{2}+\psi\right)}{\cos \frac{\vartheta}{2}}
$$

und aus der zweiten Formel (IO, 7) folgt wegen $\cos \frac{\vartheta}{2}=\mathrm{I}+O\left(\vartheta^{2}\right)$ und $\cos \left(\psi+\frac{\vartheta}{2}\right)-\cos \psi=\left(\cos \frac{\vartheta}{2}-1\right) \cos \psi-\sin \frac{\vartheta}{2} \sin \psi=O(\vartheta)$ die Behauptung unmittelbar.

\section{$\S 3$. Der Randverzerrungssatz.}

II. Formulierung des Randverzerrungssatzes. Satz IV. Es möge $w=f(z)$ ein schlichtes einfach zusammenhängendes Gebiet $G_{1}$ in der z-Ebene auf ein schlichtes Gebiet $G$ in der w-Ebene konform abbilden. Der Rand des Gebietes $G_{1}$ möge in einem Punkte $z_{0}$ eine Ecke von der Öffnung $\gamma_{1}\left(0<\gamma_{1} \leqq 2 \pi\right)$ nach dem Innern von $G_{1}$ bilden. Im entsprechenden Punkte $w_{0}$ möge der Rand des Gebietes $G$ gleichfalls eine Ecke von der öffnung $\gamma(0 \leqq \gamma \leqq 2 \pi)$ bilden. Dann gilt 
Über den Habitus der konformen Abbildung am Rande des Abbildungsbereiches. 101

$$
\frac{f^{\prime}(z)}{\frac{f(z)-w_{0}}{z-z_{0}}}=I+\left(z-z_{0}\right) g^{\prime}(z) ; \quad g(z)=\lg \frac{f(z)-w_{0}}{z-z_{0}}
$$

$$
\left(z-z_{0}\right) g^{\prime}(z) \rightarrow \frac{\gamma-\gamma_{1}}{\gamma_{1}}
$$

$$
\frac{\frac{f^{\prime}(z)}{f(z)-w_{0}}}{z-z_{0}} \rightarrow \frac{\gamma}{\gamma_{1}}
$$

wobei die Formeln (I I, 2) und (I I,3) für $z \rightarrow z_{0}$ gelten, wenn $z$ aus dem Innern von $G_{1}$ im Winkel gegen $z_{0}$ strebt. ${ }^{15}$

Die Randverzerrungsformel (I I, I) ergibt sich in allen Fällen unmittelbar aus

$$
f(z)=w_{0}+\left(z-z_{0}\right) e^{g(z)}
$$

durch Differentiation. Andererseits sind offenbar die Formeln ( I I, 2) und (I I, 3) äquivalent.

12. Beweis unter zwei speziellen Annahmen. Wir nehmen zuerst an, dass $G_{1}$ identisch mit $K_{z}$ ist und dass die Funktion

$$
\arg \frac{f(z)-w_{0}}{z-z_{0}}
$$

bei stetiger Fortsetzung im Innern von $G$ gleichmässig beschränkt bleibt. Unter diesen beiden Annahmen soll der Beweis des Satzes IV in dieser Nummer erbracht werden.

Offenbar darf dabei ohne Beschränkung der Allgemeinheit vorausgesetzt werden, dass $z_{0}=\mathrm{I}, w_{0}=0$ ist und die innere Winkelhalbierende des Randwinkels von $G$ bei $w_{0}$ in die negative reelle Axe weist, so dass die beiden Tangenten an den Rand von $G$ im Nullpunkt, vom Nullpunkt aus durchlaufen, mit der positiven reellen Axe die Winkel $\pi-\frac{\gamma}{2}$ bezw. $\pi+\frac{\gamma}{2}$ bilden.

Nun folgt aus der Voraussetzung über $\gamma$, dass bei geeigneter Wahl der Bestimmung von $(12, \mathrm{I})$ in $K_{z}$ für $\vartheta \downarrow$ o bzw. $\vartheta \uparrow o$

$$
\arg \frac{f\left(e^{i 9}\right)-w_{0}}{z-\mathrm{I}}
$$

${ }^{15}$ Für $\gamma=\gamma_{1}=\pi, G_{1}=K_{z}$ findet sich die Formel (I I, 3) in VISSER (2), p. 34, (Satz 7). 
gegen die Werte $\pi-\frac{\gamma}{2}-\frac{\pi}{2}=\frac{\pi}{2}-\frac{\gamma}{2}$ bzw. $\pi+\frac{\gamma}{2}-\frac{3 \pi}{2}=-\left(\frac{\pi}{2}-\frac{\gamma}{2}\right)$. konvergiert. Daher ergibt sich, dass bei geeigneter Wahl der Bestimmung von $g(z)$ die Randfunktion $\chi_{1}(\vartheta)$ von $\mathfrak{R} \frac{\mathrm{I}}{i} g(z)$ in der Nähe des Nullpunktes für $\vartheta \rightarrow 0$ rechtseitig gegen $\frac{\pi}{2}-\frac{\gamma}{2}$ und linkseitig gegen $-\left(\frac{\pi}{2}-\frac{\gamma}{2}\right)$ konvergiert.

Andererseits besitzt nach Satz II die Funktion

$$
\Re i \frac{\gamma-\pi}{\pi} g_{0}(z)
$$

eine Randfunktion, die für $\vartheta \downarrow 0$ bzw. $\vartheta \uparrow \circ$ die. gleichen Grenzwerte $\frac{\pi}{2}-\frac{\gamma}{2}$ bzw. $-\left(\frac{\pi}{2}-\frac{\gamma}{2}\right)$ wie $\Re \frac{I}{i} g(z)$ hat. Daher besitzt der Realteil der Funktion

$$
g^{*}(z)=\frac{\mathrm{I}}{i} g(z)-i \frac{\gamma-\pi}{\pi} g_{0}(z)
$$

eine für $z=\mathrm{I}$ stetige und in $K_{z}$ absolut beschränkte Randfunktion, sodass nach Satz I (vgl. die Bemerkungen in Nr. 9) ( $\mathrm{I}-|z|) g^{* \prime}(z)$ gegen o konvergiert, wenn $z$ allseitig in $K_{z}$ gegen I strebt. Daher folgt nach (10,5), wenn $z$ im Winkel aus dem Innern von $K_{z}$ gegen I strebt,

$$
g^{\prime}(z)=\frac{\pi-\gamma}{\pi} g_{0}^{\prime}(z)+o\left(\frac{\mathrm{I}}{\mathrm{I}-z}\right),
$$

oder endlich nach $(\mathrm{I} O, 4)$ für $n=\mathrm{I}$

$$
g^{\prime}(z)=\frac{\gamma-\pi}{\pi} \frac{\mathrm{I}}{z-\mathrm{I}}+o\left(\frac{\mathrm{I}}{\mathrm{I}-z}\right) .
$$

13. Zuendeführung des Beweises von Satz $I V$. Es sei nun noch immer $G_{1}$ identisch mit $K_{z}$, dagegen möge (12, I) nicht mehr bei stetiger Fortsetzung ins Innere von $K_{z}$ gleichmässig beschränkt bleiben. Es sei dann $G^{*}$ ein einfach zusammenhängendes Gebiet, das auf $K_{z}$ abbildbar ist, $G$ enthält und den beiden folgenden Bedingungen genügt:

Erstens: Ist $w=H(z)$ die Abbildungsfunktion von $G^{*}$ auf $K_{z}$, so bleibt

$$
\arg \frac{H(z)-w_{0}}{z-z_{0}}
$$

bei stetiger Fortsetzung in $K_{z}$ absolut beschränkt. 
Über den Habitus der konformen Abbildung am Rande des Abbildungsbereiches. 103

Zweitens: Ist $U$ eine hinreichend kleine Umgebung von $z_{0}$ auf $E_{z}$, und sind $M, M^{*}$ die bei unseren Abbildungen von $G, G^{*}$ auf $K_{z}$ der Randmenge $U$ von $K_{z}$ resp. entsprechenden Folgen von Randelementen von $G$ bezw. $G^{*}$, so sind $M$ und $M^{*}$ identisch und werden im gleichen Sinne durchlaufen - mit andern Worten, die Ränder von $G$ und $G^{*}$ fallen in der Umgebung des betreffenden Randpunktes $w_{0}$ zusammen.

Bei der Abbildung $w=H(z)$ möge $G$ auf ein Teilgebiet $K^{\prime}$ von $K_{z}$ abgebildet werden. Der Rand von $K^{\prime}$ enthält offenbar den Kreisbogen $U$. Es sei nun $w_{1}$ der Punkt von $G$, der bei der Abbildung $w==f(z)$ dem Mittelpunkt von $K_{z}$ entspricht, und es sei $z_{1}$ der Punkt von $K^{\prime}$, in den $w_{1}$ durch die Abbildung $w=H(z)$ übergeführt wird. Es sei nun $\varphi(z)$ die in $K_{z}$ reguläre Funktion, die $K_{z}$ auf $K^{\prime}$ so abbildet, dass $z=0$ in $z_{1}$ übergeführt wird und $z_{0}$ in sich übergeht. Dann gilt offenbar

$$
f(z)=H(\varphi(z))
$$

Nun ist bekanntlich $\varphi(z)$ im Innern des Kreisbogens $U$ regulär und hat dort eine von o verschiedene Ableitung. Daher gilt

$$
\frac{\varphi^{\prime}(z)}{\frac{\varphi(z)-z_{0}}{z-z_{0}}} \rightarrow \mathrm{I}
$$

wenn $z$ aus $K_{z}$ im Winkel gegen $z_{0}$ strebt. Setzt man aber $\zeta=\varphi(z)$, so strebt dann auch $\zeta$ aus $K_{z}$ im Winkel gegen $z_{0}$. Dann gilt aber nach dem in Nr. I2 bewiesenen

$$
\frac{H^{\prime}(\zeta)}{\frac{H(\zeta)-z_{0}}{\zeta-z_{0}}} \rightarrow \frac{\gamma}{\pi}
$$

Multiplizieren wir nun die beiden Formeln (I3, I) und (I3,2), so ergibt sich offenbar $(I I, 3)$, womit also der Satz IV für $G_{1} \equiv K_{z}$ bewiesen sein wird, sobald die Existenz eines Gebietes $G^{*}$ mit den obigen Eigenschaften feststeht. Ein solches Gebiet $G^{*}$ kann man aber z. B. wie folgt herstellen: Es sei $U$ eine hinreichend kleine Umgebung von $z_{0}$ auf $E_{z}$. Zeichnet man nun in der $w$-Ebene zwei Winkelräume von der Öffnung $\frac{\pi}{2}$, deren Winkelhalbierende resp. die Halbtangenten an den Rand von $G$ in $w_{0}$ sind, so folgt aus der Existenz dieser Halbtangenten in $w_{0}$, dass, wenn $U$ klein genug ist, die den Punkten von $U$ entsprechenden Rand- 
elemente von $G$ innerhalb dieser Winkelräume enthalten sind. Ist daher $V$ die Menge der dann den Punkten von $U$ entsprechenden Randelemente und ist $G^{*}$ das $G$ enthaltende einfach zusammenhängende Gebiet, das aus der $w$-Ebene nach Entfernung aller Punkte von $V$ entsteht, so genügt $G^{*}$ allen unseren Forderungen, da die Schwankung von arg $\left(w-w_{0}\right)$ auf $V$ bei stetiger Fortsetzung absolut höchstens gleich $\frac{\pi}{2}$ ist. - Damit ist nunmehr der Satz IV unter der Annahme $G_{1} \equiv K_{z}$ bewiesen.

Es ist noch von Interesse hervorzuheben, dass in einem sehr allgemeinen Spezialfall das Gebiet $G^{*}$ gleichmässig für alle Punkte eines Randbogens von $G$ gebildet werden kann. Denn es sei $\bar{\Gamma}$ ein im Rand von $G$ als freies Randstück enthaltener Jordanbogen, inklusive seiner Endpunkte, der die Ebene nicht zerlegt; $\Gamma$ sei der offene Jordanbogen, der aus den inneren Punkten von $\bar{\Gamma}$ besteht und es sei endlich $\Gamma^{\prime}$ ein abgeschlossener 'Teilbogen von $\Gamma$. Es seien $\bar{M}, M^{\prime}$ die Bögen von $E_{z}$ die $\bar{\Gamma}, \Gamma^{\prime}$ entsprechen. Durchläuft nun $z_{0}$ alle Punkte von $\boldsymbol{M}^{\prime}$ und $w_{0}$ die entsprechenden Punkte von $\Gamma^{\prime}$, so kann man jedesmal als das Gebiet $G^{*}$ das Gebiet annehmen, das aus der $w$-Ebene nach Entfernung von $\bar{\Gamma}$ entsteht, sofern man über das Verhalten der Funktion $f(z)$ auf $E_{z}$ die Zusatzvoraussetzung macht, dass das Argument von $f(z)-f\left(z_{0}\right)$ für alle $z_{0}$ auf $M^{\prime}$ und $z$ auf $\bar{M}$ gleichmässig absolut beschränkt bleibt, sobald $z \neq z_{0}$ hinreichend nahe an $z_{0}$ liegt. Das heisst also, sofern es zwei positive Konstanten $C, \delta<\mathrm{I}$ gibt, derart, dass

$$
\left|\arg \left(f(z)-f\left(z_{0}\right)\right)\right|<C
$$

gilt, sobald $z_{0}$ auf $M^{\prime}$ und $z$ auf $\bar{M}$ liegen und $\left|z-z_{0}\right|<\delta$ ist. Dabei muss natürlich $\arg \left(f(z)-f\left(z_{0}\right)\right)$ stetig in die beiden in $z_{0}$ aneinanderstossenden und in diesem Punkte offenen Teilstücke von $\bar{M}$ fortgesetzt werden bei geeigneter, von einem $z_{0}$ zum andern eventuell variablen Festlegung des Zweiges. (Mit andern Worten: es handelt sich um die Schwankung von $\arg \left(f(z)-f\left(z_{0}\right)\right)$ auf den beiden Stücken von $\bar{M}$.)

Um nun zu beweisen, dass unser Gebiet $G^{*}$ die erste beim obigen Beweis postulierte Eigenschaft besitzt, genügt es zu zeigen, dass unter unseren Voraussetzungen es eine positive Konstante $C^{*}$ gibt, derart, dass

$$
\left|\arg \left(f(z)-f\left(z_{0}\right)\right)\right|<C^{*}
$$

gilt, für alle $z_{0}$ auf $M^{\prime}$ und alle $z \neq z_{0}$ auf $\bar{M}$, wenn auf jedem der Teilstücke 
Über den Habitus der konformen Abbildung am Rande des Abbildungsbereiches. 105 von $\bar{M}$ die gleiche Bestimmung des Arguments angenommen wird, wie in der Formel $($ I 3,3$)$.

Wäre dies aber nun nicht richtig, so müsste es zwei Punktfolgen $z_{0}^{(n)}, z^{(n)}$, $n=\mathrm{I}, 2, \ldots$ geben, wo $z_{0}^{(n)}$ in $M^{\prime}, z^{(n)}$ in $\bar{M}$ liegen und $\left|\arg \left(f\left(z^{(n)}\right)-f\left(z_{0}^{(n)}\right)\right)\right|>n$ ist. Wir können dann offenbar ferner voraussetzen, dass $z^{(n)} \rightarrow \zeta, z_{0}^{(n)} \rightarrow \zeta_{0}$ gilt, wo $\zeta, \zeta_{0}$ in $\bar{M}$ bzw. $M^{\prime}$ liegen. Es sei nun $N>2 C$ und so gross, dass für $n>N$ bereits

$$
\left|z^{(n)}-\zeta\right|<\frac{\delta}{4}, \quad\left|z_{0}^{(n)}-\zeta_{0}\right|<\frac{\delta}{4}
$$

gilt; dann liegt kein $z^{(n)}$ für $n>N$ im Bereich $\left|z-\zeta_{0}\right|<\frac{\delta}{2}$, da sonst $\mid \arg \left(f\left(z^{(n)}\right)-f\left(z_{0}^{(n)}\right) \mid<C<n\right.$ wäre. Entfernt man daher aus $\bar{M}$ die $\frac{\delta}{2}-\mathrm{Um}$ gebung von $\zeta_{0}$, so müssen alle Punkte $z^{(n)}$ mit $n>N$ auf einem und demselben der beiden Kreisbögen $\boldsymbol{M}_{\mathbf{1}}, \boldsymbol{M}_{2}$ liegen, in die $\overline{\boldsymbol{M}}$ zerfällt, etwa auf $\boldsymbol{M}_{\boldsymbol{1}}$, wobei derjenige der beiden Punkte $\zeta_{0} e^{ \pm i \frac{\delta}{2}}$, der auf $M_{1}$ liegt, mit $\zeta_{1}$ bezeichnet sei. Es sei nun der Bildbogen von $\boldsymbol{M}_{1}$ in der $w$.Ebene mit $\boldsymbol{I}_{1}$ bezeichnet und es seien die Bildpunkte von $\zeta_{0}, \zeta, \zeta_{1}, z_{0}^{(n)}, z^{(n)}$ in der $w$-Ebene

$$
\eta_{0}, \eta, \eta_{1}, w_{0}^{(n)}, w^{(n)} \text {. }
$$

Dann folgt aus der Ungleichung $(13,3)$, dass die Ordnung des zwischen $\eta_{1}$ und $w^{(n)}$ enthaltenen Bogens von $\Gamma_{1}$ in bezug auf $w_{0}^{(n)}$ grösser als $n-C$ ist und daher ins Unendliche konvergiert, wenn $n$ über alle Grenzen wächst, während dabei $w_{0}^{(n)}$ gegen den Punkt $\eta_{0}$ geht, der ausserhalb $\Gamma_{1}$ liegt. Dies ist aber unmöglich, wie aus dem folgenden Hilfssatz folgt:

Hilfssatz. Es sei $J(w=\varphi(t), 0 \leqq t \leqq 1)$ ein Jordanbogen in der w-Ebene, $w_{0}$ ein nicht auf J liegender Punkt. Dann gibt es zwei positive Zahlen $D, \varepsilon$, derart, dass die Ordnung jedes in $J$ enthaltenen Jordanbogens in bezug auf einen beliebigen Punkt aus der $\varepsilon$-Umgebung von $w_{0}$ absolut genommen unterhalb $D$ bleibt.

Beweis des Hilfssatzes. Es sei $d$ die Distanz des Punktes $w_{0}$ von der abgeschlossenen Punktmenge der Punkte von $J$. Wir wählen $\varepsilon=\frac{d}{2}$. Wegen der gleichmässigen Stetigkeit von $\varphi(t)$ für $0 \leqq t \leqq$ I gibt es ein positives $\delta$, so dass für $\mathrm{o} \leqq t<t^{\prime} \leqq \mathrm{I}$ und $t^{\prime}-t<\delta$ stets

ist.

$$
\left|\varphi\left(t^{\prime}\right)-\varphi(t)\right|<\frac{d}{4}
$$

14-34472. Acta mathematica. 64. Imprimé le 30 octobre 1934. 
Ist nun

$$
\mathrm{o}=t_{0}<t_{\mathbf{1}}<\cdots<t_{n}=\mathrm{I}, t_{v}-t_{v-1}<\delta, \nu=\mathbf{I}, 2, \ldots, n
$$

so ist der ganze Bogen von $J$ zwischen $t_{v-1}$ und $t_{v}$ im Kreise um den Punkt $\varphi\left(t_{v-1}\right)$ mit dem Radius $\frac{d}{4}$ enthalten. Da aber die Distanz von $\varphi\left(t_{v-1}\right)$ von jedem Punkt der $\frac{d}{2}$-Umgebung von $w_{0}$ nicht kleiner als $\frac{d}{2}$ ist, hat dieser Teilbogen in Bezug auf jeden Punkt dieser Ungebung höchstens die Ordnung $\frac{\pi}{6}$, und da dasselbe auch für jedes Teilstück eines solchen Teilbogens gilt, ist die Ordnung jedes in $J$ enthaltenen Jordanbogens in Bezug auf jeden Punkt der $\frac{d}{2}$-Umgebung von $w_{0}$ höchstens $n \frac{\pi}{6}=D$, w. z. b. w.

Um uns endlich von der Annahme $G_{1} \equiv K_{z}$ zu befreien und damit den Satz IV unter den allgemeinsten Voraussetzungen zu beweisen, ist zu beachten, dass nach dem bereits Bewiesenen der Satz IV sowohl auf die Abbildungsfunktion von $K_{z}$ auf $G_{1}$ als auch auf die Abbildungsfunktion von $K_{z}$ auf $G$ angewandt werden kann. Aus der Gestalt der Formel (I I, 3) ergibt sich aber dann, dass diese Formel auch für die Abbildungsfunktion von $G_{1}$ auf $G$ gilt - sofern noch bewiesen wird, dass bei der Abbildung von $K_{z}$ auf $G_{1}$ eine Winkelumgebung von $z_{0}$ in $K_{z}$ in einen Bereich übergeht, der eine Winkelumgebung des entsprechenden Punktes von $G_{1}$ enthält und in einer solchen Winkelumgebung enthalten ist - und umgekehrt. Diese Tatsache wird aber in Nr. I 5 als eine unmittelbare Folgerung des Randverzerrungssatzes erkannt werden. Und zwar wird dabei für den Fall, dass eines der Gebiete mit $K_{z}$ identisch ist, auch der Randverzerrungssatz nur in diesem Falle gebraucht werden, der ja dann im Obigen bereits vollständig bewiesen ist. Damit wird aber auch der Beweis des Satzes IV im allgemeinen Falle erbracht worden sein.

14. Die Winkelstetigkeit der Drehung am Rande. Satz V. Unter den Voraussetzungen des Satzes IV gilt für $\gamma_{1}>0, \gamma>0$ :

$$
\arg \frac{f(z)-w_{0}}{z-z_{0}}=\left(\frac{\gamma}{\gamma_{1}}-\mathrm{I}\right) \arg \left(z-z_{0}\right)+c+\varepsilon\left(z-z_{0}\right),
$$

$$
\arg f^{\prime}(z)=\left(\frac{\gamma}{\gamma_{1}}-\mathrm{I}\right) \arg \left(z-z_{0}\right)+c+\varepsilon_{1}\left(z-z_{0}\right)
$$


Über den Habitus der konformen Abbildung am Rande des Abbildungsbereiches. 107

$$
\arg f^{\prime}(z)-\arg \frac{f(z)-w_{0}}{z-z_{0}}=\varepsilon_{2}\left(z-z_{0}\right)
$$

wo die Konstante $c$ dadwrch festgelegt wird, dass die Richtung der Winkelhalbierenden der Ecke von $G_{1}$ bei $z_{0}$ in die Richtung der Winkelhalbierenden der Ecke von $G$ bei $w_{0}$ ïbergeht, und $\varepsilon\left(z-z_{0}\right), \varepsilon_{1}\left(z-z_{0}\right), \varepsilon_{2}\left(z-z_{0}\right)$ gegen Null konvergieren, wenn $z$ aus dem Innern von $G_{1}$ im Winkel gegen $z_{0}$ strebt; $\varepsilon\left(z-z_{0}\right)$ konvergiert gegen Null auch bei allseitiger Konvergenz von $z$ aus $G_{1}$ gegen $z_{0}$. - (14, I) gilt auch fiur $\gamma=0$.

Beim Beweis der obigen Behauptungen darf ohne Beschränkung der Allgemeinheit vorausgesetzt werden, dass erstens $\gamma_{1}=\pi$ und $G_{1}=K_{z}$ ist, da man wiederum die Abbildung von $G_{1}$ auf $G$ »auf dem Umwege über $K_{z} \gg$ durchführen kann; dass zweitens $z_{0}=\mathrm{I}, w_{0}=\mathrm{o}$ ist und die Winkelhalbierende der Ecke (bzw. die entsprechende Spitzentangente für $\gamma=0$ ) von $G$ bei $w_{0}$ in die negative reelle Axe weist. Dann gilt für das Argument von $\frac{f(z)-w_{0}}{\left(z-z_{0}\right){ }^{\gamma / \pi}}$, wenn $z$ gegen $z_{0}=$ I längs $E_{z}$ geht, bei geeigneter Wahl der in $K_{z}$ stetigen Argumente

$$
\underset{z \rightarrow z_{0}}{\arg } \frac{f(z)-w_{0}}{\left(z-z_{0}\right)^{\gamma / \pi}} \rightarrow \pi-\frac{\gamma}{2}-\frac{\gamma}{2}=\pi+\frac{\gamma}{2}-\frac{3 \pi}{2} \frac{\gamma}{\pi}=\pi-\gamma
$$

Da aber die Funktion $\arg \frac{f(z)-w_{0}}{\left(z-z_{0}\right)^{\gamma / \pi}}$ in $K_{z}$ in der Umgebung von $z_{0}=$ I eine beschränkte Potentialfunktion ist, gilt $(14,4)$ auch, wenn $z$ in $K_{z}$ allseitig gegen $z_{0}=\mathrm{I}$ konvergiert. Dabei ist aber

$$
\arg \left(z-z_{0}\right)^{\frac{\gamma}{\pi}}=\frac{\gamma}{\pi} \arg \left(z-z_{0}\right), \quad \frac{\pi}{2}<\arg \left(z-z_{0}\right)<\frac{3 \pi}{2} .
$$

Daraus folgt erstens, wenn $z$ aus $K_{z}$ allseitig gegen I strebt,

$$
\arg \left(f(z)-w_{0}\right)=\frac{\gamma}{\pi} \arg \left(z-z_{0}\right)+\pi-\gamma+\varepsilon\left(z-z_{0}\right)
$$

wo $\varepsilon\left(z-z_{0}\right)$ nach o konvergiert. Danach gilt $(\mathrm{I} 4, \mathrm{I})$ für $\gamma \geqq 0$. Zweitens aber liefert die Formel $(1 \mathrm{I}, 3)$ des Randverzerrungssatzes für $\gamma>0$, wenn dort rechts und links die Argumente verglichen werden,

$$
\arg f^{\prime}(z)-\arg \frac{f(z)-w_{0}}{z-z_{0}} \rightarrow 0,
$$


wenn eine geeignete Bestimmung von $\arg f^{\prime}(z)$ festgelegt wird und $z$ aus $K_{z}$ im Winkel gegen I geht. Dies ist aber gerade (I 4,3$)$, und aus (I4,3) und (I4, I) folgt (14, 2) unmittelbar.

Für $\gamma=\gamma_{1}$ ergibt sich aus $(14,2)$ insbesondere, dass $\arg f^{\prime}(z)$ bei Annäherung im Winkel an $z_{0}$ einem Grenzwert zustrebt, eine Tatsache, die deshalb besonders interessant ist, weil ein Grenzwert von $f(z)$ selbst bekanntlich nicht einmal zu existieren braucht, wenn die in $z_{0}$ und $w_{0}$ zusammenstossenden Randstücke dort stetige Tangenten besitzen.

I5. Bemerkungen über die Bedeutung des Satzes $V$. In der Formel (14, I) steckt die » Winkelproportionalität bei unserer Abbildung im Punkte $z_{0}$ bzw. $w_{0}$. Dies bedeutet: Sind $L_{1}, L_{2}$ zwei in $G_{1}$ verlaufende und in $z_{0}$ einmündende Jordanbogen, die in $z_{0}$ Tangenten besitzen, so sind die Bilder von $L_{1}, L_{2}$ ganz analog beschaffene Kurven $L_{1}^{\prime}, L_{2}^{\prime}$, und der Winkel, den $L_{1}^{\prime}, L_{2}^{\prime}$ in $w_{0}$ miteinander bilden, ist gleich dem Winkel der Kurvenbogen $L_{1}, L_{2}$ in $z_{0}$, multipliziert mit $\frac{\gamma}{\gamma_{1}}$. Für $\gamma=\gamma_{1}$ haben wir die Winkeltreue.

Wir haben zwar beim Beweis des Randverzerrungssatzes von dieser Winkelproportionalität bereits Gebrauch gemacht, indessen nur um den allgemeinen Fall auf den Fall $G_{1} \equiv K_{z}$ zurückzuführen. Daher enthält unsere Herleitung der Winkelproportionalität für $G_{1} \equiv K_{z}$ sicher keinen Zirkelschluss. Beim Beweis des Randverzerrungssatzes wurde aber von der Winkelproportionalität nur für den speziellen Fall $G_{1} \equiv K_{z}$ Gebrauch gemacht. - Aus der Winkelproportionalität bei der Abbildung von $G_{1}$ auf $G$ folgt nun unmittelbar: Konvergiert eine Punktfolge $z_{v}$ aus $G_{1}$ im Winkel gegen $z_{0}$, so konvergiert auch die Bildpunktmenge $w_{v}$ aus $G$ im Winkel gegen $w_{0}$; liegen alle $z_{v}$ von einem $\nu$ an in einer Dreiecksumgebung von $z_{0}$, so liegen auch alle $w_{v}$ von einem $\nu$ an in einer Dreiecksumgebung von $w_{0}$.

Nach dem Obigen wird die Drehung eines vom Punkt $z_{0}$ ausgehenden und innerhalb des Tangentenwinkels der Ecke bei $z_{0}$ liegenden Linienelements durch $(\mathrm{I} 4, \mathrm{I})$ geliefert. Aus $(\mathrm{I} 4,3)$ folgt, dass auch $\arg f^{\prime}(z)$ denselben Grenzwert wie $\arg \frac{f(z)-w_{0}}{z-z_{0}}$ besitzt, wenn $z$ in $G_{1}$ längs einer, jenes Linienelement tangierenden Kurve gegen $z_{0}$ strebt. Durch $\arg f^{\prime}(z)$ wird aber die Drehung der Linienelemente in $G_{1}$ und daher insbesondere längs jener Kurve geliefert. Daraus folgt, dass eine in $z_{0}$ einmündende Kurve $C_{z}$, die in $z_{0}$ eine stetige Tangente 
Über den Habitus der konformen Abbildung am Rande des Abbildungsbereiches. 109 besitzt und deren Tangentenrichtung in $z_{0}$ innerhalb des Tangentenwinkels liegt, wiederum in eine solche Kurve $C_{w}$ übergeht.

Machen wir nun allgemein die Annahme, dass die Kurve $C_{z}$ im Punkte $z_{0}$ eine $L$-Tangente besitzt, und liegt die Tangente an $C_{z}$ in $z_{0}$ innerhalb des Tangentenwinkels der Ecke in $z_{0}$, so folgt nunmehr aus dem am Schluss von Nr. 5 Gesagten, dass auch die Bildkurve $C_{w}$ im Punkte $w_{0}$ eine $L$-Tangente besitzt.

Für $\gamma=\gamma_{1}$ kann der Inhalt von $(\mathrm{I} 4,3)$ folgendermassen geometrisch umschrieben werden: Man betrachte eine Winkelumgebung $D$ von $z_{0}$ in $G_{1}$, und es seien $z_{1}, z_{2}$ zwei Punkte aus $D, w_{1}, w_{2}$ ihre Bildpunkte in $G$. Man betrachte die Dreiecke $\Delta_{z}=z_{0} z_{1} z_{2}, \boldsymbol{A}_{w}=w_{0} w_{1} w_{2}$, und es seien $\alpha_{0}, \alpha_{1}, \alpha_{2}, \beta_{0}, \beta_{1}, \beta_{2}$ ihre Ecken bei $z_{0}, z_{1}, z_{2}, w_{0}, w_{1}, w_{2}$. Dann erfährt die Ecke von $\Delta_{z}$ bei $z_{0}$ die durch $\operatorname{Lim}_{z \rightarrow z_{0}} \arg \frac{f(z)-w_{0}}{z-z_{0}}$ gegebene Drehung, während die Drehung der Winkel bei $z_{1}, z_{2}$ durch $\arg f^{\prime}\left(z_{1}\right)$, arg $f^{\prime}\left(z_{2}\right)$ gegeben ist. Und aus der Formel $(14,2)$ folgt, wie wir zeigen werden, dass für innerhalb $D$ gegen $z_{0}$ konvergierende $z_{1}, z_{2}$ die Dreiecke $\mathcal{A}_{z}, \boldsymbol{A}_{w}$ in bezug auf die Winkel $\gg$ in der Grenze» ähnlich sind, d. h.

$$
\beta_{0}-\alpha_{0} \rightarrow \mathrm{o}, \beta_{1}-\alpha_{1} \rightarrow \mathrm{o}, \beta_{2}-\alpha_{2} \rightarrow 0
$$

ist. Dies ist namentlich dann wichtig, wenn die Winkel bei $z_{0}, z_{1}, z_{2}$ oberhalb einer positiven Schranke bleiben. Den Beweis von $(15, I)$ führen wir hier zunächst unter der speziellen Voraussetzung, dass $D$ eine Dreiecksumgebung ist, so dass das Innere von $\dot{A}_{z}$, bzw. $\Delta_{w}$ in $G_{1}$ bzw. $G$ enthalten ist, sobald $z_{1}$ und $z_{2}$ nahe genug bei $z_{0}$ liegen. Der Beweis im allgemeinen Falle wird später in der Num. mer 25 (Satz IX der Nr. 24) erbracht werden.

In der Tat: entsprechen den Strecken $z_{0} z_{1}, z_{1} z_{2}, z_{2} z_{0}$, in der $w$-Ebene die Kurvenbögen $C_{2}, C_{0}, C_{1}$, so folgt aus dem Rolleschen Satz, dass die Strecken $w_{0} w_{1}, w_{1} w_{2}, w_{2} w_{0}$ parallel sind $\mathrm{zu}$ den Richtungen von Tangenten an $C_{2}, C_{0}, C_{1}$ in gewissen Punkten, die etwa $\zeta_{2}, \zeta_{0}, \zeta_{1}$ innerhalb der Strecken $z_{0} z_{1}, z_{1} z_{2}, z_{2} z_{0}$ entsprechen. Nach $(14,2)$ ergeben sich daher die Richtungen der drei Seiten von $\boldsymbol{A}_{w}$ aus den Richtungen der entsprechenden Seiten von $\boldsymbol{\Delta}_{z}$ durch Drehungen um Winkel, die sich von der Konstanten $c$ in $(14,2)$ jeweils um $\varepsilon_{1}\left(\zeta_{2}-z_{0}\right)$, $\varepsilon_{1}\left(\zeta_{0}-z_{0}\right), \varepsilon_{1}\left(\zeta_{1}-z_{0}\right)$ unterscheiden. Daraus folgt aber $(15,1)$ unmittelbar.

Es sei noch bemerkt, dass, wenn die Punkte $z_{0}, z_{1}, z_{2}$ auf einer Geraden liegen und etwa $z_{1}$ zwischen $z_{0}$ und $z_{2}$ liegt, die Winkel $\alpha_{0}, \alpha_{1}, \alpha_{2}$ die Werte $\circ, \pi, o$ haben. Analoges gilt in der w.Ebene. 
16. Der Randverzerrungssatz unter der Annahme der Winkelproportionalität. Wir haben in Nr. I I vorausgesetzt, dass $G$ und $G_{1}$ in $w_{0}, z_{0}$ Ecken besitzen. Nun lässt sich bekanntlich die Winkelproportionalität - bei Annäherung im Winkel - auch unter gewissen allgemeineren Voraussetzungen herleiten. ${ }^{16}$ Es ist daher von Interesse zu zeigen, dass der Randverzerrungssatz und die Behauptungen des Satzes $\mathrm{V}$ bereits dann in einem bestimmten Umfang gelten, wenn nur bekannt ist, dass die Abbildung von $G_{1}$ auf $G$ im Punkte $z_{0}$ bei Annäherung im Winkel winkelproportional ist. Wir behaupten nämlich:

Satz $I V^{\circ}$. Es möge $w=f(z)$ ein Gebiet $G_{1}$ in der $z$-Ebene mit einem erreichbaren Randpunkt $z_{0}$ und dem Rand $R_{1}$ auf ein Gebiet $G$ in der w-Ebene mit dem Rand $R$ abbilden, wobei $z_{0}$ einem gleichfalls erreichbaren Randpunkt $w_{0}$ entsprechen möge. Es seien $L_{1}^{\prime}, L_{1}^{\prime \prime}$ zwei auf $G_{1}+R_{1}$ verlaufende Jordanbogen, die in $z_{0}$ einmünden und dort Tangenten $s_{1}^{\prime}, s_{1}^{\prime \prime}$ besitzen, derart, dass in der Nähe von $z_{0}$ zwischen $L_{1}^{\prime}, L_{1}^{\prime \prime}$ nur Punkte von $G_{1}$ liegen. Der innere Winkel zwischen $s_{1}^{\prime}$ und $s_{1}^{\prime \prime}$ sei $\gamma_{1}>0$. Es mögen $L_{1}^{\prime}, L_{1}^{\prime \prime}$ durch $w=f(z)$ auf zwei Jordanbogen $L^{\prime}, L^{\prime \prime}$ abgebildet werden, die in $w_{0}$ einmünden und dort Tangenten $s^{\prime}, s^{\prime \prime}$ besitzen, die miteinander den innern Winkel $\gamma$ bilden. Ist dann $D$ eine Winkelumgebung von $z_{0}$ in $G_{1}$, die zwischen $L_{1}^{\prime}, L_{1}^{\prime \prime}$ liegt, deren Begrenzung jedoch mit $s_{1}^{\prime}, s_{1}^{\prime \prime}$ nur den Punkt $z_{0}$ gemeinsam hat, so gelten, wenn z gegen $z_{0}$ durch das Innere von $D$ strebt, die Formeln $(1 \mathrm{I}, \mathrm{I}),(\mathrm{I}$ I , 2), (I I, 3), (I 4, I) und, wenn $\gamma>0$ ist, (I 4, 2), (I 4, 3). Die Formel (14, I) gilt dabei auch dann, wenn $z$ gegen $z_{0}$ strebt und dabei nur der Bedingung unterworfen bleibt, zwischen $L_{1}^{\prime}, L_{1}^{\prime \prime} z u$ bleiben. Insbesondere besitzt also $\arg f^{\prime}(z)$ fï $\cdot \gamma=\gamma_{1}$ einen Grenzwert, wenn $z$ ïber $D$, d.h. im Winkel, gegen $z_{0}$ strebt. ${ }^{17}$

Zum Beweis verbinde man einen von $z_{0}$ verschiedenen Punkt von $L_{1}^{\prime}$ mit einem von $z_{0}$ verschiedenen Punkt von $L_{1}^{\prime \prime}$ durch einen Jordanbogen $C$, der, bis eventuell auf seine Endpunkte, ganz innerhalb von $G_{1}$ verläuft und weder mit $L_{1}^{\prime}$ noch mit $L_{1}^{\prime \prime}$ Punkte gemeinsam hat. Durch $C$ wird aus dem zwischen $L_{1}^{\prime}, L_{1}^{\prime \prime}$ liegenden Stück von $G_{1}$ ein Gebiet $G_{1}^{*}$ herausgeschnitten, dessen Berandung aus $C$ und Teilbogen von $L_{1}^{\prime}$ und $L_{1}^{\prime \prime}$ besteht und in $z_{0}$ eine Ecke mit der innern Öffnung $\gamma_{1}$ besitzt. $G_{1}^{*}$ wird nach unserer Voraussetzung durch $w=f(z)$ auf ein

16 Es lässt sich sogar in gewissen Fällen, wo der Rand von $G$ in $w_{0}$ nicht einmal eine Tangente besitzt und $G_{1} \equiv K_{z}$ ist, die Existenz einer von o und $\infty$ verschiedenen Winkelableitung beweisen. Carathéodory (3), pp. 4, I7-I8, (2), p. 97 ; Ahlfors (I), p. 40.

${ }^{17} \mathrm{Im}$ Fall $G_{1} \equiv K_{z}$, wenn über die Voraussetzungen von Satz $I V^{\circ}$ hinaus $f(z)$ in $z_{0}$ eine (eventuell anch unendliche) Winkelderivierte besitzt, findet sich die Formel (II,3) bei VISSER (2), p. 35. (Satz 8). 
Über den Habitus der konformen Abbildung am Rande des Abbildungsbereiches. 111 Teilgebiet $G^{*}$ von $G$ abgebildet, dessen Berandung im Bildpunkte $w_{0}$ von $z_{0}$ eine Ecke der Öffnung $\gamma$ hat. Wendet man nun die Sätze IV, V auf die Gebiete $G_{1}^{*}, G^{*}$ an, so ergeben sich unsere Behauptungen unmittelbar.

\section{$\S$ 4. Bemerkungen zum Lindelöfschen Satz.}

I7. Der Lindelöfsche Satz. Die in der Formel $(\mathrm{I} 4,2)$ für $\gamma=-\gamma_{1}$ enthaltene Existenz des Grenzwertes von $\arg f^{\prime}(z)$ bei Konvergenz im Winkel gegen $z_{0}$ ist für den speziellen Fall, dass $G_{1} \equiv K_{z}$ ist und der Rand von $G$ in $w_{0}$ eine $L$-Tangente besitzt, bereits von Herrn E. Lindelöf festgestellt worden, und zwar hat sich in diesem Falle die Existenz von Lim arg $f^{\prime}(z)$ sogar bei allseitiger Annäherung an $z_{0}$ aus $K_{z}$ ergeben. Offenbar ist hier die Beschränkung $G_{1}=K_{z}$ unwesentlich, da man ja die Abbildung von $G_{1}$ auf $G$ immer auf dem Umwege über $K_{z}$ ausführen kann. Wir können daher den Lindelöfschen Satz folgendermassen formulieren:

Satz VI. (Der Lindelöfsche Satz.) Es möge $w=f(z)$ ein.schlichtes einfach zusammenhängendes Gebiet $G_{1}$ der z-Ebene auf ein schlichtes Gebiet $G$ in der w-Ebene konform abbilden. Der Rand $R_{1}$ von $G_{1}$ möge in einem Randpunkt $z_{0}$ mit einer $L$.Tangente versehen sein, und dasselbe möge für den Rand $R$ von $G$ im Bildpunkt $w_{0}$ von $z_{0}$ gelten. Dann strebt arg $f^{\prime}(z)$ gegen einen endlichen Grenzwert, wenn $z$ aus dem Innern von $G_{1}$ allseitig gegen $z_{0}$ konvergiert.

Der Lindelöfsche Satz gilt insbesondere, wenn die Ränder von $G_{1}, G$ in $z_{0}$ bzw. $w_{0}$ stetige Tangenten besitzen. Für diesen Fall lässt er sich mit der gleichen Methode herleiten wie oben $\left(\mathrm{I}_{4}, 2\right)$, wenn man beim Beweis des Satzes I auf die Gleichmässigkeit der Konvergenz auf geeigneter $\vartheta$-Menge achtet. Um indessen den Lindelöfschen Satz für den Fall einer $L$-Tangente mit herzuleiten, muss die ganze Grundlage der Untersuchung erweitert werden, und er wird sich daher bei unserer Methode erst im zweiten Teil dieser Abhandlung (Nr. 6I) ergeben. Um aber Wiederholungen zu vermeiden, sollen bereits im ersten Teil dieser Abhandlung verschiedene Folgerungen aus diesem Satz mitentwickelt werden, und wir wollen daher zuerst einige Verallgemeinerungen des Lindelöfschen Satzes angeben.

Es sei zu allererst bemerkt, dass im Falle, wo $G_{1}$ und $G$ in $z_{0}$ bzw. $w_{0}$ $L$-Tangenten besitzen, die in $\mathrm{Nr}$. I 5 angegebene Folgerung aus der Existenz von Lim $\arg f^{\prime}(z)$ über die Abbildung von Kurven mit $L$-Tangenten offenbar 
sogar bei allseitiger Konvergenz gegen $z_{0}$ gilt. Ist also $C_{z}$ eine auf der Menge $G_{1}+R_{1}$ verlaufende Jordankurve, die in $z_{0}$ mit einer $L$-Tangente mündet, so mündet auch ihre auf $G+R$ verlaufende Bildkurve in $w_{0}$ mit einer $L$-Tangente. U̇ber die Formel ( $\left.{ }_{5}, \mathrm{I}\right)$ in diesem Fall vgl. den Satz IX.

I 8. Übertragung des Lindelöfschen Satzes auf L-Tangentenecken. Die Behauptung des Lindelöfschen Satzes besagt einfach, dass $(14,2)$ und daher alle drei Formeln $(\mathrm{I} 4, \mathrm{I}),(\mathrm{I} 4,2),\left(\mathrm{I}_{4}, 3\right)$ bei allseitiger Konvergenz von $z$ gegen $z_{0}$ gelten, und in dieser Formulierung lässt sich der Lindelöfsche Satz auch auf den Fall übertragen, dass $G$ und $G_{1}$ in $w_{0}$ bzw. $z_{0}$ zwar nicht notwendig $L$-Tangenten, wohl aber Ecken von den Öffnungen $\gamma>0, \gamma_{1}>0$ bilden, derart, dass die beiden im Punkte $w_{0}$ zusammenstossenden Jordanbogen dort $L$-Tangenten besitzen und das Gleiche für die in $z_{0}$ zusammenstossenden Randstücke von $G_{1}$ gilt. Beim Beweis genügt es offenbar wiederum vorauszusetzen, dass $\gamma_{1}=\pi$ ist. Für $\gamma_{1}=\pi$ aber lässt sich die zu beweisende Tatsache wohl am einfachsten feststellen, wenn man durch eine gebrochene Potenztransformation die Öffnung des Winkels bei $w_{0}$ gleich $x$ macht. In der Tat können wir ohne Beschränkung der Allgemeinheit voraussetzen, dass $w_{0}=0$ ist und die Winkelhalbierende der inneren Ecke von $G$ bei $w_{0}$ in die negative reelle Axe weist. Dann wird das Gebiet $G$ durch die Transformation $w^{\prime}=w^{\frac{\pi}{\gamma}}$ bei geeigneter Wahl der Bestimmung von $w^{\frac{\pi}{\gamma}}$ in ein solches Gebiet $G^{\prime}$ übergeführt, dass bei $w^{\prime}=0$ eine Ecke der Öffnung $\pi, d . h$. eine Tangente vorliegt. Und da unsere Transformation winkelproportional ist, so folgt aus dem am Schlusse der $\mathrm{Nr} .5$ Gesagten, dass auch die beiden im Punkte $w^{\prime}=0$ zusammenstossenden Randstücke von $G^{\prime}$ dort $L$-Tangenten besitzen und daher also das ganze Randstück von $G^{\prime}$ in $w^{\prime}=0$ eine $L$-Tangente hat. Für das Gebiet $G^{\prime}$ gilt also

$$
\arg \frac{d w^{\prime}}{d z}=c+\varepsilon_{0}\left(z-z_{0}\right)
$$

wo $\varepsilon_{0}\left(z-z_{0}\right)$ bei allseitiger Konvergenz von $z$ aus $G_{1}$ gegen $z_{0}$ gegen o strebt. Daraus folgt weiter, wegen $(\mathrm{I} 4, \mathrm{I})$, auf $w^{\prime}$ als Funktion von $z$ angewandt:

$$
\begin{aligned}
& \arg w=\frac{\gamma}{\pi} \arg \left(z-z_{0}\right)+\frac{\gamma}{\pi} c+\frac{\gamma}{\pi} \varepsilon\left(z-z_{0}\right), \\
& \arg f^{\prime}(z)=\left(\mathrm{I}-\frac{\pi}{\gamma}\right) \arg w+c+\varepsilon_{0}\left(z-z_{0}\right), \\
& \arg f^{\prime}(z)=\left(\frac{\gamma}{\pi}-\mathrm{I}\right) \arg \left(z-z_{0}\right)+\frac{\gamma}{\pi} c+\left(\frac{\gamma}{\pi}-\mathrm{I}\right) \varepsilon\left(z-z_{0}\right)+\varepsilon_{0}\left(z-z_{0}\right) .
\end{aligned}
$$


Über den Habitus der konformen Abbildung am Rande des Abbildungsbereiches. 113

Dies sind aber die Formeln $(14,1),(14,2)$ für $\gamma_{1}=\pi$, in denen nunmehr in der Tat, wie behauptet, die $\varepsilon$-Glieder bei allseitiger Konvergenz von $z$ gegen $z_{0}$ gegen 0 streben. ${ }^{18}$ Und dasselbe gilt natürlich auch für ( 14,3 ).

Die damit bewiesene Übertragung des Lindelöfschen Satzes auf $L$-Tangentenecken ist im allgemeineren Satz der nächsten Nr. enthalten.

19. Der Lindelöfsche Satz für halbseitige Konvergenz. Wir wollen endlich beweisen, dass der Lindelöfsche Satz in der verallgemeinerten Formulierung der Nr. I 8 »zur Hälfte» erbalten bleibt, wenn unter obigen Voraussetzungen schon der eine der in $z_{0}$ zusammenstossenden Randbogen von $G_{1}$ - er sei mit $C_{1}$ bezeichnet - und der entsprechende Randbogen $C$ von $G$ in $z_{0}$ bzw. $w_{0} L$-Tangenten besitzen. In diesem Falle darf nämlich $z$ halbseitig am Bogen $C_{1}$ gegen $z_{0}$ gehen. Wir formulieren den Satz wie folgt:

Satz VII. Es möge $w=f(z)$ ein schlichtes einfach zusammenhängendes Gebiet $G_{1}$ der $z$-Ebene mit einer Ecke von der Öffnung $\gamma_{1}>0$ in einem Randpunkte $z_{0}$ auf ein schlichtes Gebiet $G$ in der $w$-Ebene konform abbilden, wobei der Ecke in $z_{0}$ eine Ecke im Bildpunkt $w_{0}$ von der Öffnung $\gamma>0$ entspricht. Es möge einer der beiden in $z_{0}$ zusammenstossenden Randbogen von $G_{1}$ - er sei mit $C_{1}$ bezeichnetin $z_{0}$ eine L-Tangente besitzen, ebenso wie sein Bildbogen $C$ im Punkte $w_{0}$. Dann gelten die Formeln (I4, I), (I 4, 2), (I4,3), wenn $z$ halbseitig am Bogen $C_{1}$ aus $G_{1}$ gegen $z_{0}$ konvergiert.

Beim Beweis darf offenbar vorausgesetzt werden, dass das Gebiet $G_{1}$ mit dem Kreissektor identisch ist, der aus dem Einheitskreis $K_{z}$ durch die beiden unter dem Winkel $\frac{\pi}{4}$ zur negativen reellen Axe geneigten Radien herausgeschnitten wird. Der Punkt $z_{0}$ sei in diesem Falle der Nullpunkt, und $C_{1}$ sei einer der beiden Begrenzungsradien von $G_{1}$. - In der Tat kann ja die Abbildung von $G_{1}$ auf $G$ »auf dem Umwege» über einen solchen Bereich stets durchgeführt werden.

Ebenso kann auch das Gebiet $G$ in geeignet spezialisierter Gestalt angenommen werden. Denn erstens kann $G$ durch ein Teilgebiet $G^{\prime}$ ersetzt werden, das zwischen den beiden in $w_{0}$ zusammenstossenden Randbogen und einem bei $w_{0}$ verlaufenden Querschnitt von $G$ liegt. Denn bekanntlich - wir haben von einer analogen Tatsache bereits oben Gebrauch gemacht - ist die Abbildungsfunktion von $G^{\prime}$ auf $G_{1}$ in der Form darstellbar $f^{\prime}(\varphi(z))$, wo $\varphi(z)$ und $\varphi^{\prime}(z)$ in

18 Der Beweis für den Lindelöfschen Fall $G_{1}=K_{z}, \gamma=\pi$ ist natürlich noch unmittelbarer. 15-34472. Acta mathematica. 64. Imprimé le 31 octobre 1934. 
$z_{0}$ und auf einer Umgebung von $z_{0}$ stetig und von o verschieden sind. Entspricht nämlich $G^{\prime}$ bei der Abbildung von $G$ auf $G_{1}$ das Teilgebiet $G_{1}^{\prime}$ des Kreissektors $G_{1}$, so vermittelt $u=\varphi(z)$ die Abbildung von $G_{1}$ auf $G_{1}^{\prime}$. Dem Randbogen $C$ möge bei $G^{\prime}$ etwa $C^{\prime}$ entsprechen. Wir verlängern nun $C^{\prime}$ längs der Tangente in $w_{0}$ und bilden ein einfach zusammenhängendes Gebiet $G^{*}$, das das Gebiet $G^{\prime}$ im Innern enthält und zu dessen Rand als freies Randstück $C^{\prime}$ mit einem Stück der verlängerten Tangente gehört, so dass also der Rand von $G^{*}$ in $w_{0}$ eine $L$-Tangente besitzt. Wird nun $G^{*}$ etwa auf die obere Hälfte des Einheitskreises in der $u$-Ebene abgebildet, derart, dass dabei $w_{0}$ in $u=0$ übergeht, so wird dabei $G^{\prime}$ auf ein Gebiet $G^{\prime \prime}$ abgebildet, in dem das Bild $C^{\prime \prime}$ von $C^{\prime}$ geradlinig ist. Und man kann bekanntlich die Abbildung von $G^{*}$ auf jenes Gebiet der $u$-Ebene so einrichten, dass einer der beiden in der reellen $u$-Axe liegenden Radien von $E_{u}$ das Bild von $C^{\prime}$ darstellt. Es genügt nun offenbar unsern Satz für das so entstandene Gebiet $G^{\prime \prime}$ zu beweisen. Wir dürfen daher von vornherein annehmen, dass das Gebiet $G$ in der oberen Halbebene liegt, dass ferner $w_{0}=0$ und $C$ eine der beiden Strecken zwischen $w=0$ and $w=I$ bzw. $w=\mathrm{o}$ und $w=-\mathrm{I}$ ist. Wird nun aber ein solches Gebiet $G$ durch $w=f(z)$ auf das vorhin angegebene Gebiet $G_{1}$ abgebildet, so entspricht dabei der geradlinigen Strecke $C$ einer der beiden Begrenzungsradien des Kreissektors $G_{1}$, nämlich $C_{1}$. Man »verdoppele» nun das Gebiet $G$ durch Spiegelung an $C$ und bezeichne das so entstehende Gebiet mit $\Gamma$; ebenso "verdoppele" man $G_{1}$ durch Spiegelung an $C_{1}$ und bezeichne die so entstehende Halbkreisscheibe mit $\Gamma_{1}$. Aus dem Schwarzschen Spiegelungsprinzip folgt aber nunmehr, dass die Funktion $f(z)$ zugleich die konforme Abbildung von $\Gamma_{1}$ auf $\Gamma$ derart vermittelt, dass dabei $z_{0}$ in $w_{0}$ übergeht. Wendet man nun auf diese Abbildung die Formeln $(14,1)$, $(14,2),(14,3)$ an, so gelten sie auf jeden Fall in einem Winkel um $C_{1}$, woraus sich unsere Behauptung unmittelbar ergibt.

20. Folgerungen aus Satz VII. Es ist nun klar, dass die am Schluss der Nr. 17 für den Fall von $L$-Tangenten angegebene Tatsache auch unter den Voraussetzungen des Satzes VII für am Randbogen $C_{1}$ halbseitig gegen $z_{0}$ konvergierende $z$ erhalten bleibt. Mit andern Worten: Es sei $C_{z}$ eine in $G_{1}$ verlaufende und in $z_{0}$ mündende einfache Kurve, die in $z_{0}$ eine $L$-Tangente besitzt und in einer an $C_{1}$ liegenden halbseitigen Umgebung von $z_{0}$ innerhalb $G_{1}$ verläuft. Dann besitzt auch ihre Bildkurve in $G$ im Punkte $w_{0}$ eine $L$-Tangente. Und hier darf $C_{z}$ auch mit $C_{1}$ Punkte gemein haben. 
Über den Habitus der konformen Abbildung am Rande des Abbildungsbereiches. 115

Ebenso gilt für $\gamma=\gamma_{1}>0$ die in $\mathrm{Nr}$. I 5 erläuterte $\gg$ Winkelähnlichkeit in der Grenze» des Dreiecks $z_{0} z_{1} z_{2}$ mit dem Dreieck $w_{0} w_{1} w_{2}$, dessen Ecken Bildpunkte von $z_{0}, z_{1}, z_{2}$ sind, wenn $z_{1}, z_{2}$ halbseitig an $C_{1}$ gegen $z_{0}$ konvergieren, $\mathrm{d}$. h. die Formel $\left({ }_{5}, \mathrm{I}\right)$ bleibt auch jetzt richtig. Wir beweisen sie hier allerdings unter speziellen Voraussetzungen und werden den allgemeinen Fall erst im Satze IX formulieren und beweisen.

Lemma $I$. Es sei $D$ unter den Voraussetzungen des Satzes VII eine halbseitige Umgebung von $z_{0}$ an $C_{1}$, deren Winkelöffinung $\gamma_{0}$ bei $z_{0}$ positiv und kleiner als $\pi$ ist. $z_{1}, z_{2}$ sei ein Paar untereinander verschiedener Punkte aus der Menge $D+C_{1}$, die gegen $z_{0}$ konvergieren, $w_{1}, w_{2}$ seien die Bildpunkte von $z_{1}$ und $z_{2}$ in $G$. Dann ist die Strecke $w_{1} w_{2}$ gegen die Strecke $z_{1} z_{2}$ um den Winkel $c+\varepsilon\left(z_{1}, z_{2}\right)$ gedreht, wo $\varepsilon\left(z_{1}, z_{2}\right)$ gegen o konvergiert, wenn $z_{1}$ und $z_{2}$ gegen $z_{0}$ streben, und $c$ die Konstante der Formel $(\mathrm{I} 4,2)$ ist.

Zum Beweis beachte man vor allem, dass, sobald $z_{1}, z_{2}$ nahe genug bei $z_{0}$ liegen, auch das Punktepaar $w_{1}, w_{2}$ innerhalb einer Halbumgebung von $w_{0}$ an $C$ bleibt, deren Winkelöffnung bei $w_{0}$ beliebig nahe an $\gamma_{0}$ und daher insbesondere positiv und kleiner als $\pi$ gewählt werden kann. Daher kann man beim Beweis die Gebiete $G$ und $G_{1}$ miteinander vertauschen. Andererseits kann man die Abbildung von $G_{1}$ auf $G$ auf dem Umwege über einen Sektor des Einheitskreises ausführen, wobei $C_{1}$ einem Radius dieses Kreises entspricht. Daher darf beim Beweis von vornherein vorausgesetzt werden, dass $C_{1}$ eine geradlinige Strecke ist.

Es sei nun $D^{*}$ eine weitere halbseitige Umgebung von $z_{0}$ an $C_{1}$, die $D$ enthält und deren Winkelöffnung bei $z_{0}$ grösser als $\gamma_{0}$ ist. Sobald $z_{1}$ und $z_{2}$ nahe genug bei $z_{0}$ liegen, bleibt dann die Strecke $z_{1} z_{2}$ sicher innerhalb $D^{*}+C_{1}$. Liegt höchstens einer der Punkte $z_{1}, z_{2}$ auf $C_{1}$, so liegen alle inneren Punkte der Strecke $z_{1} z_{2}$ innerhalb $D^{*}$. Ist $\Gamma$ die Bildkurve der Strecke $z_{1} z_{2}$ in der $w$-Ebene, so hat ihre Sehne $w_{1} w_{2}$ nach dem Rolleschen Satz die gleiche Richtung wie die Tangente an $\Gamma$ in einem innern Punkt dieses Bogens, der einem innern Punkte $\zeta$ der Strecke $z_{0} z_{1}$ entspricht. Daher ergibt sich nach $(14,2)$ die Richtung von $w_{1} w_{2}$ aus der Richtung von $z_{1} z_{2}$ durch Drehung um den Winkel $c+\varepsilon_{1}\left(\zeta-z_{0}\right)$, woraus unsere Behauptung ohne weiteres folgt.

Liegen aber beide Punkte $z_{1}, z_{2}$ auf $C_{1}$, so ist die Richtung von $z_{1} z_{2}$ zugleich diejenige von $C_{1}$. Die Strecke $w_{1} w_{2}$ ist dann eine Sehne an die Kurve $C$; die Richtung von $w_{1} w_{2}$ konvergiert daher nach der Annahme, dass $C$ in $w_{0}$ eine 
$L$-Tangente besitzt, gegen die Richtung der Tangente an $C$ in $w_{0}$. In diesem Falle aber ergibt sich unsere Behauptung unmittelbar aus dem Satze VII.

Aus Lemma I folgt nunmehr:

Lemma 2. Wird über die Voraussetzungen von Lemma I hinaus vorausgesetzt, dass die Punkte $z_{1}, z_{2}$ nicht nur voneinander sondern auch von $z_{0}$ verschieden sind, so gelten die Formeln (I5, I), wenn $z_{1}, z_{2}$ ïber $D+C_{1}$ gegen $z_{0}$ konvergieren.

Denn zum Beweis genügt es, das Lemma I auf jedes der drei Punktepaare $z_{0}, z_{1} ; z_{0}, z_{2} ; z_{1}, z_{2}$ anzuwenden.

Endlich sei noch folgendes bewiesen:

Zusatz I zum Satz VII. Es mögen in der Formulierung des Satzes VII die Jordanbogen $C$ und $C_{1}$ durchweg mit Tangenten versehen sein, die in den Punkten $z_{0}$ bzw. $w_{0}$ L-Tangenten sind, und es sei $\gamma=\gamma_{1}$. Dann bilden die längs des Bogens $C_{1}$ im Winkel existierenden Grenzwerte von arg $f^{\prime}(z)$ eine Funktion des Punktes auf $C_{1}$, die in $z_{0}$ stetig ist. Ist in der Tat $\alpha$ der Grenzwert von $\arg f^{\prime}(z)$ in $z_{0}$ und $\varepsilon$ eine beliebig kleine positive Zahl, so gibt es eine halbseitige Umgebung $H$ von $z_{0}$ an $C_{1}$, derart, dass für alle $z$ aus $H\left|\arg f^{\prime}(z)-\alpha\right|<\varepsilon$ ist. Ist dann $C_{1}^{*}$ ein an $z_{0}$ anstossendes Stiick von $C_{1}$, das zum Rand von $H$ gehört, und ist $z^{\prime}$ irgend ein innerer Punkt von $C_{1}^{*}$, so liegt die Innennormale auf $C_{1}$ in $z^{\prime}$ zunächst in $H$. Daher genügt der längs der Normalen sicher existierende Grenzwert $\arg f^{\prime}\left(z^{\prime}\right)$ von $\arg f^{\prime}(z)$ in $z^{\prime}$ der Ungleichung

$$
\left|\arg f^{\prime}\left(z^{\prime}\right)-\alpha\right| \leqq \varepsilon,
$$

wie behauptet. Insbesondere folgt:

Zusatz 2 zum Satz VII. Haben unter den Voraussetzungen des Satzes VI zwei einander entsprechende Jordansche Randbogen $C_{1}$ und $C$ von $G_{1} b z w . G$ durchweg sich stetig drehende Tangenten, so ist die Grenzfunktion von $\arg f^{\prime}(z)$ eine stetige Funktion auf dem Bogen $C_{1}{ }^{19}$

\section{$\S$ 5. Relative Konformität bei der Eckenabbildung.}

21. Die Längentreue im Kleinen.

Satz VIII. Es sei unter den Voraussetzungen des Satzes IV $\gamma>0, \gamma_{1}>0$; $c_{1}, c_{2}$ seien zwei beliebige positive Konstanten mit $c_{1}<c_{2} . \quad D$ sei eine Winkelumgebung von $z_{0}$ in $G_{1}$.

${ }^{19}$ Vgl. für diese Formulierung Warschawski (I), p. 406. 
Über den Habitus der konformen Abbildung am Rande des Abbildungsbereiches. 117

a) Ist dann $z_{1}, z_{2}, z_{1} \neq z_{2}$ ein Punktepaar aus $D$ mit

so gilt

$$
c_{1} \leqq\left|\frac{z_{1}-z_{0}}{z_{2}-z_{0}}\right| \leqq c_{2}
$$

$$
\begin{gathered}
\frac{f\left(z_{1}\right)-w_{0}}{f\left(z_{2}\right)-w_{0}} \sim\left(\frac{z_{1}-z_{0}}{z_{2}-z_{0}}\right)^{\frac{\gamma}{\gamma_{1}}} \\
\frac{f^{\prime}\left(z_{2}\right)}{f^{\prime}\left(z_{1}\right)} \sim\left(\frac{z_{2}-z_{0}}{z_{2}-z_{0}}\right)^{\frac{\gamma}{\gamma_{1}}-1}
\end{gathered}
$$

wenn $z_{1}$ und $z_{2}$ über $D$, d.h. im Winkel, gegen $z_{0}$ konvergieren.

b) Wird im Falle a) die Voraussetzung (21, I) ersetzt durch

$\left(21, I^{\circ}\right)$

$$
\frac{z_{1}-z_{0}}{z_{2}-z_{0}} \rightarrow \text { o, bzw. } \frac{z_{1}-z_{0}}{z_{2}-z_{0}} \rightarrow \infty
$$

so sind die Formeln $(2 \mathrm{I}, 2),(2 \mathrm{I}, 3)$ zu ersetzen durch

$$
\frac{f\left(z_{1}\right)-w_{0}}{f\left(z_{2}\right)-w_{0}} \rightarrow \text { o, bzw. } \frac{f\left(z_{1}\right)-w_{0}}{f\left(z_{2}\right)-w_{0}} \rightarrow \infty .^{20}
$$

c) Sind $w_{1}, w_{2}$ die den Punkten $z_{1}, z_{2}$ aus $D$ entsprechenden Punkte aus $G$, so ist Bedingung (2 I, I) äquivalent mit

$$
d_{1} \leqq\left|\frac{w_{1}-w_{0}}{w_{2}-w_{0}}\right| \leqq d_{2}
$$

für positive Konstanten $d_{1}, d_{2}$, wobei die Äquivalenz so zu verstehen ist, dass zu festen $c_{1}, c_{2}$ solche positive $d_{1}, d_{2}$ gefunden werden können, dass (21,4) eine Folge von (2I, I) ist, und umgekehrt, zu festen positiven $d_{1}, d_{2}$ positive $c_{1}, c_{2}$ derart wählbar sind, dass $(2 \mathrm{I}, \mathrm{I})$ aus $(2 \mathrm{I}, 4)$ folgt. Ebenso ist jede Bedingung $\left(2 \mathrm{I}, \mathrm{I}^{\circ}\right)$ äquivalent mit der entsprechenden Bedingung $\left(2 \mathrm{I}, 2^{\circ}\right)$.

${ }^{20}$ Man kann offenbar die auf $(2 \mathrm{I}, 1),(2 \mathrm{I}, 2),\left(2 \mathrm{I}, \mathrm{I}^{\circ}\right),\left(2 \mathrm{I}, 2^{\circ}\right)$ bezüglichen Behauptungen dahin zusammenfassen, dass wenn $z_{1}$ und $z_{2}$ im Winkel gegen $z_{0}$ konvergieren und $\frac{z_{1}-z_{0}}{z_{2}-z_{0}} \rightarrow \lambda$ ist, dann auch $\frac{f\left(z_{1}\right)-w_{0}}{f\left(z_{2}\right)-w_{0}} \rightarrow \lambda^{\frac{\gamma}{\gamma_{1}}}$ gilt. Es ist leicht $z$ u sehen, dass hieraus die ganze Behauptung (2I, 2) unter der Voraussetzung $(2 \mathrm{I}, \mathrm{r})$ unmittelbar hergeleitet werden kann. 
d) Hat ein an $z_{0}$ anstossendes Randstück $C_{1}$ von $G_{1}$ in $z_{0}$ eine L-Tangente, ebenso wie sein Bildbogen $C$ in $w_{0}$ und gilt fiir $z_{1}, z_{2}$ aus einer halbseitigen Umgebung $H$ von $z_{0}$ an $C_{1}$ in $G_{1}(2 \mathrm{I}, \mathrm{I})$, bzw. (2 $\left.\mathrm{I}^{\circ} \mathrm{I}^{\circ}\right)$, so darf in den Behauptungen a), b), c), die Formel $(2 \mathrm{I}, 3)$ ausgenommen, die Winkelumgebung $D$ durch $H$ ersetzt werden. Und dabei dürfen $z_{1}$ und $z_{2}$ auch auf $C_{1}$ liegen. - Haben alle vier in $z_{0}$ und $w_{0}$ zusammenstossenden Randbogen von $G_{1}$ und $G$ dort L-Tangenten, so darf in dieser Formulierung die Umgebung $H$ durch eine allseitige Omgebung von $z_{0}$ in $G_{1}$ ersetzt werden, zu der dabei auch noch die Randpunkte von $G_{1}$, die zugleich zum Rand dieser Umgebung gehören, hinzugezählt werden können.

e) Gelten anstatt den Voraussetzungen des Satzes IV die Voraussetzungen des Satzes $I V^{\circ}$ der $\mathrm{Nr}$. I6, so bleiben die Behauptungen a), b), c) richtig, wenn die Winkelumgebung $D$ den in der Formulierung von $I V^{\circ}$ angegebenen Bedingungen genügt. ${ }^{21}$

22. Beweis von $(2 \mathrm{I}, 2)$ bzw. $\left(2 \mathrm{I}, 2^{\circ}\right)$ für $\gamma=\gamma_{1}$. Es sei zunächst bemerkt, dass $(2 I, 3)$ aus $(2 I, 2)$ wegen $(I I, 3)$ ohne weiteres folgt, so dass im Falle a) nur $(2 \mathrm{I}, 2) \mathrm{zu}$ beweisen ist. Ferner ist die Relation (2 I, 4) offenbar eine unmittelbare Folge aus $(2 \mathrm{I}, 2)$ und $(2 \mathrm{I}, \mathrm{I})$. Beachtet man, dass nach dem in Nr. I 5 Gesagten für $z_{1} \prec D, z_{2} \prec D$, sobald $z_{1}$ und $z_{2}$ nahe genug bei $z_{0}$ liegen, auch $w_{1}, w_{2}$ in einer Winkelumgebung von $w_{0}$ in $G$ liegen, so folgt aus $(2 \mathrm{I}, 4)$ umgekehrt $(2 \mathrm{I}, \mathrm{I})$, wenn man die Rollen von $z$ und $w$ vertauscht.

Wir haben daher nur $(2 \mathrm{I}, 2),\left(2 \mathrm{r}, 2^{\circ}\right)$ unfer entsprechenden Voraussetzungen $\mathrm{zu}$ beweisen. - Es seien nun wieder $\alpha_{0}, \alpha_{1}, \alpha_{2} ; \beta_{0}, \beta_{1}, \beta_{2}$ die Winkel der Dreiecke $z_{0} z_{1} z_{2}, w_{0} w_{1} w_{2}$ resp. bei $z_{0}, z_{1}, z_{2} ; w_{0}, w_{1}, w_{2}$. Beim Beweis von $(2 \mathrm{I}, 2)$ und $\left(2 \mathrm{I}, 2^{\circ}\right)$ in den Fällen a), b) dürfen wir annehmen, dass $z_{1}, z_{2}$ in einer und derselben Dreiecksumgebung von $z_{0}$ in $G_{1}$ liegen - da man sonst zwischen $z_{1}$ und $z_{2}$ nur endlich viele Punkte einzuschalten braucht, derart, dass zwei aufeinanderfolgende dieser Punkte in einer und derselben Dreiecksumgebung von $z_{0}$ in $G_{1}$ liegen. (Man beachte, dass die zu beweisende Behauptung in dem Sinne transitiv ist, dass, wenn sie für $z_{1}, z_{2}$ und $z_{2}, z_{3}$ gilt, sie auch für $z_{1}, z_{3}$ richtig ist). Daher dürfen wir die Gültigkeit von ( $\mathrm{I}_{5}, \mathrm{I}$ ) voraussetzen - für den Fall einer Dreiecksumgebung haben wir ja in Nr. 15 diese Formel bewiesen. - Ferner kann durch Einschaltung weiterer Punkte in endlicher Anzahl erreicht werden, dass der

21 Für $G_{1} \equiv K_{z}, \gamma=\pi$ und wenn $z_{1}$ und $z_{2}$ auf einem in Bezug auf $z_{0}$ symmetrischen Orthogonalkreis von $E_{z}$ liegen, ist die dann aus $(2 \mathrm{I}, 2)$ folgende Relation $\frac{\left|f\left(z_{1}\right)-w_{0}\right|}{\left|f\left(z_{2}\right)-w_{0}\right|} \rightarrow$ I mit einer in Visser (2), p. 32 (Satz 4) hergeleiteten äquivalent. 
Über den Habitus der konformen Abbildung am Rande des Abbildungsbereiches. 119 Winkel $\alpha_{0}$ zwischen $\delta$ und $\frac{\delta}{4}$ bleibt, wo $\delta$ eine beliebig aber fest gewählte positive Zahl mit $\delta<\frac{\gamma}{2}, \delta<\frac{\pi}{2}$ ist. $^{22}$ Dann gilt $\pi-\delta \leqq \alpha_{1}+\alpha_{2} \leqq \pi-\frac{\delta}{4}$. Nun liefert der Sinussatz

$$
\left|\frac{z_{1}-z_{0}}{z_{2}-z_{0}}\right|=\frac{\sin \alpha_{2}}{\sin \alpha_{1}}
$$

Aus (2 I, I) folgt daher, dass beide Winkel $\alpha_{1}, \alpha_{2}$ (kleiner als $\pi-\frac{\delta}{4}$ und) grösser als eine positive Schranke bleiben, die nur von $c_{1}, c_{2}$ abhängt. Daher bleiben wegen (I 5, I) auch die Winkel $\beta_{1}, \beta_{2}$ oberhalb einer positiven Schranke und überschreiten $\pi-\frac{\delta}{4}$ nicht, sobald $z_{1}, z_{2}$ nahe genug bei $z_{0}$ liegen, woraus wegen (22, I) nach dem Sinussatz folgt

$$
\left|\frac{f\left(z_{1}\right)-w_{0}}{f\left(z_{2}\right)-w_{0}}\right|=\frac{\sin \beta_{2}}{\sin \beta_{1}} \sim \frac{\sin \alpha_{2}}{\sin \alpha_{1}}=\left|\frac{z_{1}-z_{0}}{z_{2}-z_{0}}\right|,
$$

w. z. b. w.

Wird aber eine der Relationen $\left(2 \mathrm{I}, \mathrm{I}^{\circ}\right)$ vorausgesetzt, so folgt, dass entweder $\alpha_{2} \rightarrow$ o gilt und $\sin \alpha_{1}$ oberhalb einer positiven Schranke bleibt, oder $\alpha_{1} \rightarrow$ o gilt und $\sin \alpha_{2}$ oberhalb einer positiven Schranke bleibt. Dann gilt also entweder

oder

$$
\left|\frac{w_{1}-w_{0}}{w_{2}-w_{0}}\right|=\frac{\sin \beta_{2}}{\sin \beta_{1}} \rightarrow 0
$$

$$
\left|\frac{w_{1}-w_{0}}{w_{2}-w_{0}}\right|=\frac{\sin \beta_{2}}{\sin \beta_{1}} \rightarrow \infty
$$

womit $\left(2 \mathrm{I}, 2^{\circ}\right)$ bewiesen ist.

Offenbar führt nun die gleiche U̇berlegung auch im Falle d) zum Ziel; wir dürfen wiederum annehmen, dass $z_{1}, z_{2}$ in einer halbseitigen Umgebung von $z_{0}$ an $C_{1}$ liegen, deren Öffnung kleiner ist als $\pi$, und zu der noch die inneren Punkte von $C_{1}$ hinzuzuzählen sind. Daher gelten auch hier die Relationen ( I5, I) nach dem im Lemma 2 der Nr. 20 Bewiesenen. Ebenso darf wieder an-

${ }^{22}$ Wenn z. B. der Winkel zwischen $z_{0} z_{1}$ und $z_{0} z_{2}$ kleiner ist als $\frac{\delta}{4}$, wähle man für $z^{\prime}$ einen solchen Punkt (ausserhalb des Winkelraumes zwischen den Halbstrahlen an $z_{0}$ durch $z_{1}$ bzw. $z_{2}$ ), dass der Winkel zwischen $z_{0} z^{\prime}$ und $z_{0} z_{1}$ etwa gleich $\frac{\delta}{2}$ ist. 
genommen werden, dass $\alpha_{0}$ zwischen $\delta$ und $\frac{\delta}{4}$ für ein geeignetes positives $\delta$ mit $\delta<\frac{\gamma}{2}, \delta<\frac{\pi}{2}$ liegt. Dann sind aber alle weiteren Schlïsse unverändert anwendbar.

Und damit ist offenbar auch der Fall erledigt, in dem alle vier in $z_{0}$ und $w_{0}$ zusammenstossenden Randbogen von $G_{1}$ und $G$ dort $L$-Tangenten besitzen. -

23. Der Fall $\gamma \geqslant \gamma_{1}$. Der Fall endlich, dass $\gamma$ und $\gamma_{1}$ untereinander (und von Null) verschieden sind, lässt sich auf den oben behandelten zurückführen, wenn man die $z$-Ebene der Transformation $u=\left(z-z_{0}\right)^{\frac{\gamma}{\gamma_{1}}}$ unterwirft. Besitzen im Falle d) einige der in $z_{0}$ zusammenstossenden Randstïcke von $G_{1}$ in diesem Punkte $L$-Tangenten, so gilt nach dem in Nr. 5 Bemerkten das Gleiche auch für das aus $G_{1}$ vermöge unserer Transformation hervorgehende Gebiet, so dass (2I, 2) bzw. $\left(2 \mathrm{I}, 2^{\circ}\right)$ auch bei halbseitiger oder allseitiger Konvergenz von $z_{1}$ und $z_{2}$ gegen $z_{0}$ gilt, wobei $z_{1}$ und $z_{2}$ auch Randpunkte von $G_{1}$ durchlaufen dürfen, dem Fall d) entsprechend. Aus dem Randverzerrungssatz ergibt sich ferner, dass gleichzeitig mit $(2 \mathrm{I}, 2)$ im Falle a) auch die Relation $(2 \mathrm{I}, 3)$ gilt. Die Behauptungen von c) ergeben sich ganz analog wie oben.

Was endlich den Fall e) anbetrifft, so ergeben sich die in diesem Falle ausgesprochenen Behauptungen unmittelbar, wenn man das zum Beweis des Satzes IV Gesagte berücksichtigt. Damit ist der Satz VIII vollständig bewiesen.

Um nun analoge Aussagen über $\frac{w_{2}-w_{1}}{w_{2}-w_{0}}$ herleiten zu können, müssen wir erst die Gültigkeit der Relationen (15, I) im allgemeinsten Falle diskutieren. Wir fassen das Resultat im Satz IX zusammen, dessen Beweis auf dem Satz VIII für $\gamma=\gamma_{1}$ beruht.

24. Winkeltreue bei der Dreiecksabbildung. Satz IX. Es sei unter den Voraussetzungen des Satzes $I V \gamma=\gamma_{1}>$ o. $D$ sei eine Winkelumgebung von $z_{0}$ in $G_{1} . z_{1}, z_{2}$ sei ein Punktepaar aus $D$, wobei $z_{0}, z_{1}, z_{2}$ untereinander verschieden sind. Es seien $w_{1}, w_{2}$ die Bildpunkte von $z_{1}, z_{2}$ in $G$ und $\alpha_{0}, \alpha_{1}, \alpha_{2} ; \beta_{0}, \beta_{1}, \beta_{2}$ seien die Winkel der Dreiecke $z_{0} z_{1} z_{2}, w_{0} w_{1} w_{2}$ resp. bei $z_{0}, z_{1}, z_{2} ; w_{0}, w_{1}, w_{2}$. Dann gilt, wenn $z_{1}, z_{2}$ gegen $z_{0}$ konvergieren,

$$
\beta_{0}-\alpha_{0} \rightarrow 0, \quad \beta_{1}-\alpha_{1} \rightarrow 0, \quad \beta_{2}-\alpha_{2} \rightarrow 0
$$


Über den Habitus der konformen Abbildung am Rande des Abbildungsbereiches. 121

Hat aber ein in $z_{0}$ mündender Randbogen $C_{1}$ von $G_{1}$ dort eine $L$-Tangente, ebenso wie sein Bildrandbogen von $G$, so darf in der obigen Behauptung $D$ durch eine halbseitige Umgebung $H$ von $z_{0}$ an $C_{1}$ ersetzt werden, wobei $z u H$ noch die inneren Punkte von $C_{1}$ hinzugezählt werden dïrfen. Haben ferner alle vier in $z_{0}$ zusammenstossenden Randbogen von $G_{1}$ und $G$ dort L-Tangenten, so bleibt (24, I) für $\gamma<2 \pi$ richtig, wenn $z_{1}, z_{2}$ gegen $z_{0}$ ïber das Innere und den Rand von $G_{1}$ konvergieren. Ist aber dann $\gamma=2 \pi$, so gilt $(24, \mathrm{I})$, wenn $z_{1}, z_{2}$ gegen $z_{0}$ ïber das Innere und den Rand von $G_{1}$ konvergieren und wenn dabei für keine Unterfolge von Punktepaaren $z_{1}, z_{2}$ mit $z_{1} \rightarrow z_{0}, z_{2} \rightarrow z_{0} \quad \alpha_{0} \rightarrow \mathrm{O},\left|\frac{z_{1}-z_{0}}{z_{2}-z_{0}}\right| \rightarrow \mathrm{I}$ gilt und zugleich die Strecke $z_{1} z_{2}$ beide in $z_{0}$ zusammenstossenden Randbogen von $G_{1}$ trifft.

25. Beweis. Wäre eine der Behauptungen unseres Satzes falsch, so liesse sich nach dem Auswahlsatz eine Folge von Punktepaaren $z_{1}, z_{2}$ mit $z_{1} \rightarrow z_{0}, z_{2} \rightarrow z_{0}$ finden, die der in Frage kommenden Bedingung für $z_{1}$ und $z_{2}$ genügt und für die eine der Differenzen $\beta_{0}-\alpha_{0}, \beta_{1}-\alpha_{1}, \beta_{2}-\alpha_{2}$ einen von Null verschiedenen Grenzwert besitzt. Und das Gleiche würde dann für jede unendliche Unterfolge einer solchen Folge gelten. Eine solche Unterfolge $z_{1}^{(v)}, z_{2}^{(\nu)}(\nu=1,2, \ldots)$ können wir nun aber nach dem Häufungsstellensatz auf jeden Fall derart herausgreifen, dass für sie erstens alle sechs Winkel $\alpha_{0}^{(v)}, \alpha_{1}^{(v)}, \alpha_{2}^{(v)} ; \beta_{0}^{(v)}, \beta_{1}^{(v)}, \beta_{2}^{(v)}$ Grenzwerte besitzen, die wir mit $A_{0}, A_{1}, A_{2} ; B_{0}, B_{1}, B_{2}$ bezeichnen wollen; dass zweitens

$$
\left|\begin{array}{l}
z_{2}^{(v)}-z_{0} \\
z_{1}^{(v)}-z_{0}
\end{array}\right| \rightarrow q_{1}, \quad\left|\frac{w_{2}^{(\nu)}-w_{0}}{w_{1}^{(\nu)}-w_{0}}\right| \rightarrow q
$$

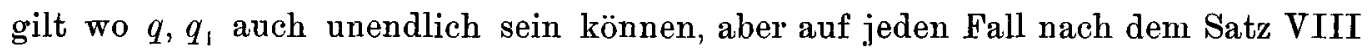
$q=q_{1}$ ist; dass drittens die Richtungen der Halbstrahlen $z_{0} z_{1}^{(v)}, z_{0} z_{2}^{(v)} ; w_{0} w_{1}^{(v)}$, $w_{0} w_{2}^{(\nu)}$ wo $w_{0}, w_{1}^{(\nu)}, w_{2}^{(\nu)}$ die Bilder von $z_{0}, z_{1}^{(v)}, z_{2}^{\langle v\rangle}$ sind, gegen die Richtungen der Halbstrahlen $\sigma_{1}^{\prime}, \sigma_{1}^{\prime \prime}$ in der $z$-Ebene bzw. $\sigma^{\prime}, \sigma^{\prime \prime}$ in der $w$-Ebene konvergieren. Und wir haben daher nur zu untersuchen, unter welchen Umständen für eine solche Folge von Punktepaaren eine der Differenzen $B_{0}-A_{0}, B_{1}-A_{1}, B_{2}-A_{2}$ von Null verschieden sein kann.

Es sei zuförderst bemerkt, dass nach $(14, \mathrm{I})$ sicher $A_{0}=B_{0}$ ist. Ist nun $A_{0}=B_{0}=\pi$, so muss offenbar $B_{1}=A_{1}=B_{2}=A_{2}=$ o sein. Es sei nun $0<A_{0}=$ $=B_{0}<\pi$. Dann verschwindet von einem $\nu$ an keiner der Winkel $\alpha_{1}^{(v)}, \beta_{1}^{(v)}, \alpha_{2}^{(v)}, \beta_{2}^{(v)}$. Der Sinussatz, angewandt auf die Dreiecke $z_{0} z_{1}^{(v)} z_{2}^{(v)}, w_{0} w_{1}^{(v)} w_{2}^{(v)}$, liefert

16-34472. Acta mathematica. 64. Imprimé le 31 octobre 1934. 
$(25,1)$

$$
\left|\frac{z_{1}^{(v)}-z_{0}}{z_{2}^{(v)}-z_{0}}\right|=\frac{\sin \alpha_{2}^{(v)}}{\sin \alpha_{1}^{(v)}} \rightarrow q, \quad\left|\frac{w_{1}^{(v)}-w_{0}}{w_{2}^{(v)}-w_{0}}\right|=\frac{\sin \beta_{3}^{(v)}}{\sin \beta_{1}^{(v)}} \rightarrow q
$$

Andererseits aber können, wegen $0<A_{1}+A_{2}=B_{1}+B_{2}=\pi-A_{0}<\pi$, $\sin A_{1}$ und $\sin A_{2}$ nicht zugleich verschwinden, ebenso wie $\sin B_{1}$ und $\sin B_{2}$. Daher gilt sicher

$$
\frac{\sin A_{2}}{\sin A_{1}}=q=\frac{\sin B_{2}}{\sin B_{1}}
$$

Und hieraus folgt $A_{1}=B_{1}, A_{2}=B_{2}$ unmittelbar für $q=0$ oder $q=\infty$. Ist aber $q$ von $O$ und $\infty$ verschieden, so folgt bekanntlich aus $(25,2)$

$$
\frac{\operatorname{tg} \frac{A_{2}-A_{1}}{2}}{\operatorname{tg} \frac{A_{2}+A_{1}}{2}}=\frac{\operatorname{tg} \frac{B_{2}-B_{1}}{2}}{\operatorname{tg} \frac{B_{2}+B_{1}}{2}}
$$

oder $\operatorname{tg} \frac{A_{2}-A_{1}}{2}=\operatorname{tg} \frac{B_{2}-B_{1}}{2}, A_{2}-A_{1}=B_{2}-B_{1}, A_{1}=B_{1}, A_{2}=B_{2}$. Es bleibt nur noch der Fall $A_{0}=B_{0}=\mathrm{o}$ zu untersuchen.

Für $A_{0}=B_{0}=0$ ist der Fall $q \neq \mathrm{I}$ sofort zu erledigen. Wir können dann ohne Beschränkung der Allgemeinheit annehmen, dass $q<\mathrm{I}$ ist. Wäre nun etwa $\mathrm{o}<A_{1}<\pi$, so würde daraus folgen, wegen $A_{2}=\pi-A_{1}-A_{0}$, nach $(25, \mathrm{I})$

$$
q=\frac{\sin A_{2}}{\sin A_{1}}=\frac{\sin \left(\pi-A_{1}\right)}{\sin A_{1}}=\mathrm{I},
$$

entgegen der Annahme. Daher muss $A_{1}$ gleich o oder $\pi$ sein, für $q<1$ also, da dann von einem $v$ an, wegen $(25, \mathrm{I})$, die $\alpha_{1}^{(v)}$ gegenüberliegende Seite des Dreiecks $z_{0} z_{1}^{(\nu)} z_{2}^{(\nu)}$ grösser als die andere an $z_{0}$ anstossende Seite ist, $A_{1}=\pi, A_{2}=0$. Genau ebenso ergibt sich $B_{1}=\pi, B_{2}=0$, womit $(24, \mathrm{I})$ auch in diesem Falle bewiesen ist.

Es sei $A_{0}=B_{0}=0, q=\mathrm{I}$. Fällt dann die gemeinsame Grenzrichtung $\sigma_{1}^{\prime}, \sigma_{1}^{\prime \prime}$ in das Innere des Eckenwinkels von $G_{1}$ bei $z_{0}$, so folgt $(24,1)$ aus dem am Schlusse von Nr. I 5 Gesagten (vgl. ( $\left.\mathrm{I}_{5}, \mathrm{I}\right)$ ). Fällt ferner die gemeinsame Richtung $\sigma_{1}^{\prime}, \sigma_{1}^{\prime \prime}$ in eine der beiden Tangentenrichtungen der Ecke von $G_{1}$ in $z_{0}$, sind aber die beiden Tangentenrichtungen dieser Ecke verschieden (ist also $\gamma_{1}<2 \pi$ ), so bleiben, wenn der zugehörige Randbogen von $G_{1}$ mit $C_{1}$ bezeichnet wird und $H$ eine Halbumgebung von $z_{0}$ an $C$ ist, $z_{1}^{(v)}, z_{2}^{(v)}$ für hinreichend grosse $\nu$ in $H+C$, 
Über den Habitus der konformen Abbildung am Rande des Abbildungsbereiches. 123

und in diesem Falle folgt die Behauptung des Satzes aus dem Lemma 2 der Nr. 20.

Es sei nun $\gamma_{1}=2 \pi$, es möge aber jede Strecke $z_{1}^{(v)} z_{2}^{(v)}$ nuv einen der beiden in $z_{0}$ zusammenstossenden Randbogen von $G_{1}$ treffen. Ferner seien $H^{\prime}, H^{\prime \prime}$ irgendwie gewählte halbseitige Umgebungen von $z_{0}$ an den in $z_{0}$ zusammenstossenden Randbogen $C_{1}^{\prime}, C_{1}^{\prime \prime}$ von $G_{1}$. Dann müssen von einem $\nu$ an beide Punkte $z_{1}^{(v)}, z_{2}^{(v)}$ in einer und derselben, vielleicht mit $v$ variablen, der beiden Mengen $H^{\prime}+C_{1}^{\prime}, H^{\prime \prime}+C_{1}^{\prime \prime}$ liegen. Nach dem Auswahlsatz dürfen wir dann annehmen, dass alle Punktepaare $z_{1}^{(v)}, z_{2}^{(v)}$ in einer festen dieser Mengen liegen, etwa in $H^{\prime}+C_{1}^{\prime}$. Dann ergibt sich aber unsere Behauptung wiederum aus dem Lemma 2 der Nr. 20.

Damit ist der Satz IX vollständig bewiesen. Was aber den in seiner Formulierung angegebenen Ausnahmefall anbetrifft, so ist es leicht, sich an einem Beispiel zu überzeugen, dass in ihm der Satz IX in der Tat nicht zu gelten braucht.

Hierzu betrachte man den Einheitskreis $K_{z}$ der $z$-Ebene, der aber längs des nach $z=$ I führenden Radius aufgeschnitten wird. Wird das so entstehende Gebiet $K_{z}^{*}$ und das analoge Gebiet in der $w$-Ebene mit $K_{w}^{*}$ bezeichnet, so bilde man $K_{z}^{*}$ auf $K_{w}^{*}$ derart ab, dass $z=0$ in $w=0$ übergeht, dass aber vom Nullpunkt verschiedene und ihm genügend benachbarte, einander gegenüberliegende Punkte auf den beiden Ufern des Einschnittes von $K_{z}^{*}$ jedesmal in zwei Randpunkte von $K_{w}^{*}$ übergehen, die nicht mehr einander gegenüberliegen. - Hierzu genügt es die Abbildungsfunktion so zu wählen, dass sie nicht die Form $w=z$ hat. -

Es seien nun $z_{1}, z_{2}$ zwei von $z_{0}$ verschiedene, einander gegenüberliegende Punkte der beiden Ufer des Einschnittes von $K_{z}^{*}$. Thre Bildpunkte in $K_{w}^{*}$ seien $w_{1}, w_{2}$ und es mögen $z_{1}, z_{2}$ so nahe beim Nullpunkt liegen, dass $w_{1}, w_{2}$ auch auf den beiden Ufern des Einschnittes von $K_{w}^{*}$ liegen. Es liege etwa $w_{1}$ näher zum Nullpunkt als $w_{2}$. Sind dann $w_{1}^{\prime}, w_{2}{ }^{\prime}$ hinreichend nahe bei $w_{1}, w_{2}$ liegende Punkte von $\boldsymbol{K}_{w}^{*}$ aus den entsprechenden Halbumgebungen von $w_{1}, w_{2}$, so unterscheiden sich die Winkel des Dreiecks o $w_{1}^{\prime} w_{2}^{\prime}$ beliebig wenig $\circ, \pi, o$. Die entsprechen. den Punkte $z_{1}^{\prime}, z_{2}{ }^{\prime}$ in der Nähe von $z_{1}, z_{2}$ lassen sich offenbar aber so wählen, dass die analogen Winkel des Dreiecks $o z_{1}^{\prime} z_{2}{ }^{\prime}$ sich beliebig wenig von $0,0, \pi$ unterscheiden.

26. Seitenähnlichkeit bei der Dreiecksabbildung. Wir sind nunmehr imstande, die dem Satz VIII entsprechenden Tatsachen über das asymptotische Verhalten der $z_{0}, w_{0}$ gegenüberliegenden Seiten der Dreiecke $z_{0} z_{1} z_{2}, w_{0} w_{1} w_{2}$ zu formulieren und $\mathrm{zu}$ beweisen. 
Satz X. Es sei unter den Voraussetzungen des Satzes $I V \gamma=\gamma_{1}>0$. c sei eine beliebige positive Konstante. $D$ sei eine Winkelumgebung von $z_{0}$ in $G_{1}$. $z_{1}, z_{2}$, $z_{1} \neq z_{2}$, sei ein Punktepaar aus $D$ mit $z_{1} \rightarrow z_{0}, z_{2} \rightarrow z_{0}$ und

$$
\left|\frac{z_{1}-z_{0}}{z_{2}-z_{0}}\right| \leqq c
$$

Es sei $w_{1}=f\left(z_{1}\right), w_{2}=f\left(z_{2}\right)$.

a) Es gilt

$(26,2)$

$$
\frac{w_{1}-w_{2}}{w_{2}-w_{0}} \sim \frac{z_{1}-z_{2}}{z_{2}-\frac{z_{0}}{z_{0}}}
$$

b) Hat ein an $z_{0}$ anstossendes Randstück $C_{1}$ von $G_{1}$ in $z_{0}$ eine L-Tangente, ebenso wie sein Bildbogen in $w_{0}$, und ist $H$ eine Halbumgebung von $C_{1}$ in $G_{1}$, so bleibt $(26,2)$ richtig, wenn die Winkelumgebung $D$ durch die Menge $H+C_{1}$ ersetzt wird, wenn dabei für keine Unterfolge von Punktepaaren $z_{1}, z_{2} \frac{z_{1}-z_{0}}{z_{2}-z_{0}} \rightarrow \mathrm{I}$ gilt.

c) Haben alle vier in $z_{0}$ und $w_{0}$ zusammenstossenden Randbogen von $G_{1}$ und $G$ dort $L$-Tangenten, so darf in der obigen Formulierung die Winkelumgebung $D$ durch eine allseitige Umgebung von $z_{0}$ in $G_{1}^{\top}$ ersetzt werden, zu der dabei auch noch die Randpunkte von $G_{1}$, die zugleich zum Rand dieser Umgebung gehören, hinzugezählt uerden können, sofern fïr keine Unterfolge von Punktepaaren $z_{1}, z_{2}$ $\frac{z_{1}-z_{0}}{z_{2}-z_{0}} \rightarrow$ I gilt

Bemerkungen. I) Legt main die Voraussetzungen des Satzes IVo zugrunde, so bleibt für $\gamma=\gamma_{1}$ die Behauptung a) des Satzes $\mathbf{X}$ richtig, wenn $D$ den in jenem Satz formulierten Bedingungen genügt. Und ebenso bleibt die Behauptung b) richtig, wenn unter $D$ eine entsprechend zu definierende halbseitige Umgebung eines der in der Formulierung von IV eingeführten Jordanbogen $L_{1}^{\prime}, L_{1}^{\prime \prime}$ verstanden wird. - In der Tat folgt aus der Formulierung des Satzes IVo, dass man in diesem Falle unsere Behauptungen ohne weiteres aus dem Satz X herleiten kann.

2) Beim Beweis von $(26,2)$ genügt es in allen Fällen des Satzes $\mathbf{X}$, nur die Äquivalenz der absoluten Beträge zu beweisen (wovon in der nächsten Nummer, beim Beweis eines Spezialfalles von a) Gebrauch gemacht werden wird): 
Über den Habitus der konformen Abbildung am Rande des Abbildungsbereiches. 125

$$
\left|\frac{\frac{w_{1}-w_{2}}{w_{2}-w_{0}}}{\frac{z_{1}-z_{2}}{z_{2}-z_{0}}}\right| \rightarrow \mathrm{I}
$$

da ja der auf die Argumente der beiden Seiten von $(26,2)$ bezïgliche Teil der Behauptung im Satze IX enthalten ist.

27. Beweis von a) für eine Dreiecksumgebung. Wir beweisen zuerst a) für den Spezialfall, dass $D$ eine Dreiecksumgebung ist und über $(26, I)$ hinaus die Voraussetzung (2 I, I) mit positiven $c_{1}, c_{2}$ gilt. Es genügt zu beweisen, dass

$(27, \mathrm{I})$

$$
\overline{\operatorname{Lim}}\left|\frac{\frac{w_{1}-w_{2}}{w_{2}-w_{0}}}{\frac{z_{1}-z_{2}}{z_{2}-z_{0}}}\right| \leqq \mathrm{I}
$$

ist, da daraus, wenn man die Gebiete $G_{1}$ und $G$ miteinander vertauscht, sich eine symmetrische Ungleichung für den Lim und damit $(26,3)$ unmittelbar ergibt. Nun ist aber die Länge der Strecke $w_{1} w_{2}$ höchstens gleich der Länge des Bildbogens von $z_{1} z_{2}$. Daher folgt

$$
\left|w_{1}-w_{2}\right| \leqq \int_{z_{1}}^{z_{2}}\left|\frac{d w}{d z}\right| d z
$$

wo geradlinig von $z_{1}$ nach $z_{2}$ zu integrieren ist. Hier lässt sich aber $\left|\frac{d w}{d z}\right|$ auf Grund des Randverzerrungssatzes (vgl. (I I, 3)) durch

$$
(1+\varepsilon)\left|\frac{w-w_{0}}{z-z_{0}}\right|
$$

abschätzen, wo $\varepsilon$, ebenso wie später $\varepsilon_{1}, \varepsilon_{2}$, eine Funktion von $z_{1}$ und $z_{2}$ ist, die mit $z_{1}-z_{0}$ und $z_{2}-z_{0}$ gleichmässig nach $\circ$ konvergiert. Daher ergibt sich nach dem ersten Mittelwertsatz der Integralrechnung

$$
\left|w_{1}-w_{2}\right| \leqq\left(\mathrm{I}+\varepsilon_{1}\right)\left|z_{1}-z_{2}\right|\left|\frac{w^{\prime}-w_{0}}{z^{\prime}-z_{0}}\right|
$$

wo $z^{\prime}$ ein Punkt der Strecke $z_{1} z_{2}$ ist und $w^{\prime}$ sein Bildpunkt. Nun ist die Be- 
dingung (2I, I) des Satzes VIII offenbar auch für das Punktepaar $z^{\prime}, z_{2}$ erfüllt, so dass in $(2 \mathrm{I}, 2) z_{1}$ durch $z^{\prime}, f\left(z_{1}\right)$ durch $w^{\prime}$ ersetzt werden kann. Daher ergibt sich weiter

$$
\left|w_{1}-w_{2}\right| \leqq\left(\mathrm{I}+\varepsilon_{1}\right)\left(\mathrm{I}+\varepsilon_{2}\right)\left|z_{1}-z_{2}\right|\left|\frac{w_{2}-w_{0}}{z_{2}-z_{0}}\right|
$$

womit $(27, \mathrm{I})$ bewiesen ist.

28. Beweis des Satzes $X$. Wenn $(26,3)$ nicht gilt, gibt es nach dem Auswahlprinzip eine den entsprechenden Voraussetzungen genügende Folge von Punktepaaren, für die die linke Seite von $(26,3)$ einen von I verschiedenen evtl. unendlichen Grenzwert hat. Und dies gilt dann auch für jede unendliche Unterfolge dieser Folge. Wir dürfen daher annehmen, dass für die Folge von Punktepaaren $z_{1}^{(v)}, z_{2}^{(v)}$ folgendes zutrifft:

I) Sind $w_{1}^{(v)}, w_{2}^{(v)}$, die Bilder von $z_{1}^{(v)}, z_{2}^{(\nu)}$, so konvergieren die sechs Winkel $\alpha_{0}^{(v)}, \alpha_{1}^{(v)}, \alpha_{2}^{(\nu)} ; \beta_{0}^{(v)}, \beta_{1}^{(\nu)}, \beta_{2}^{(\nu)}$ der Dreiecke $z_{0} z_{1}^{(\nu)} z_{2}^{(v)}, w_{0} w_{1}^{(v)} w_{2}^{(v)}$ resp. gegen $A_{0}, A_{1}, A_{2} ;$ $B_{0}, B_{1}, B_{2}$, wo nach $\left(24\right.$, I) $A_{0}=B_{0}, A_{1}=B_{1}, A_{2}=B_{2}$ ist.

$$
\text { 2) (28, I }) \quad \frac{z_{1}^{(\nu)}-z_{0}}{z_{2}^{(\nu)}-z_{0}} \rightarrow q, \quad \frac{w_{1}^{(\nu)}-w_{0}}{w_{2}^{(v)}-w_{0}} \rightarrow q_{1}, q=q_{1}
$$

wo $q$ wegen $(26, \mathrm{I})$ von $\infty$ verschieden ist. Dass die beiden Grenzwerte bei 2) identisch sind, folgt aus dem Satz VIII und $A_{0}=B_{0}$.

3) Die Richtungen der Halbstrahlen $z_{0} z_{1}^{(v)}, z_{0} z_{2}^{(v)} ; w_{0} w_{1}^{(v)}, w_{0} w_{2}^{(\nu)}$ konvergieren gegen die Richtungen der Halbstrahlen $\sigma_{1}^{\prime}, \sigma_{1}^{\prime \prime}$ in der $z$-Ebene bzw. $\sigma^{\prime}, \sigma^{\prime \prime}$ in der $w$-Ebene.

Ist nun $q \neq \mathrm{I}$, so folgt aus den Formeln

$$
\begin{aligned}
& \frac{w_{2}^{(v)}-w_{1}^{(v)}}{w_{2}^{(v)}-w_{0}}=\mathrm{I}-\frac{w_{1}^{(v)}-w_{0}}{w_{2}^{(v)}-w_{0}} \rightarrow \mathrm{I}-q, \\
& \frac{z_{2}^{(v)}-z_{1}^{(v)}}{z_{2}^{(v)}-z_{0}}=\mathrm{I}-\frac{z_{1}^{(v)}-z_{0}}{z_{2}^{(v)}-z_{0}} \rightarrow \mathrm{I}-q
\end{aligned}
$$

$(26,2)$ unmittelbar. Für $q=\mathrm{I}$, aber kommt nur der Fall a) in Betracht. Hier muss dann nach 3) der Halbstrahl $\sigma_{1}^{\prime}=\sigma_{1}^{\prime \prime}$ in einer Winkel-, daher auch in einer Dreiecksumgebung von $z_{0}$ liegen, sodass in diesem Falle $(26,3)$ aus dem in Nr. 27 Bewiesenen folgt. Damit ist der Beweis des Satzes X vollendet. 
Über den Habitus der konformen Abbildung am Rande des Abbildungsbereiches. 127 29. Eine Bemerkung zum Satz $X$. Wird unter den Annahmen des Satzes X $(29,1)$

$$
\frac{z_{1}-z_{2}}{z_{2}-z_{0}} \rightarrow 0
$$

vorausgesetzt, so folgt, wenn man auf beiden Seiten dieser Relation I addiert und den Satz VIII berücksichtigt,

$$
\frac{z_{1}-z_{0}}{z_{2}-z_{0}} \rightarrow \mathrm{I}, \quad \frac{w_{1}-w_{0}}{w_{2}-w_{0}} \rightarrow \mathrm{I}
$$

und daher, wenn von den beiden Seiten der letzten Relation I abgezogen wird,

$$
\frac{w_{1}-w_{2}}{w_{2}-w_{0}} \rightarrow 0
$$

Dabei darf hierin das Punktepaar $z_{1}, z_{2}$, je nach den Annahmen über die Beschaffenheit des Randes, über eine Winkelumgebung bzw. eine halbseitige oder allseitjge Umgebung von $z_{0}$ gegen $z_{0}$ konvergieren.

\section{§ 6. Vereinzelte Korollare zum Randverzerrungssatz.}

30. Satz von der Winkelableitung. Satz XI. Unter den Voraussetzungen des Satzes IV sei $\gamma>0$ und es möge der Grenzwert

$$
(30, \mathrm{I}) \quad \operatorname{Lim} \frac{f(z)-w_{0}}{z-z_{0}}=\lambda
$$

existieren, wenn $z$ aus dem Innern von $G_{1}$ im Winkel gegen $z_{0}$ konvergiert, mag dabei $\lambda$ endlich oder unendlich sein. Dann gilt

$$
f^{\prime}(z) \rightarrow \frac{\gamma}{\gamma_{1}} \lambda
$$

wenn $z$ im Winkel gegen $z_{0}$ strebt. Umgekehrt folgt $(30, \mathrm{I})$ aus $(30,2) .{ }^{23}$

Der Satz XI ist eine unmittelbare Folge der Formel $(1,3)$ des Randverzerrungssatzes. Dass $(30,2)$ aus $(30,1)$ folgt, ist für endliche $\lambda$ und $\gamma_{1}=\pi$ ein Spezialfall eines allgemeineren Satzes von VALIRoN, dessen Beweis von AнLfors

${ }^{23}$ Für $G_{1}=E_{z}, \gamma=\gamma_{1}=\pi$ findet sich dieser Satz bei VIsser (2), p. 37 unten. 
ausführlich entwickelt wurde. ${ }^{24}$ Für $\lambda=\infty$ dagegen sind die beiden angegebenen 'Tatsachen neu.

Wenn $\lambda$ endlich ist, folgt aus

$$
\frac{f(z)-w_{0}}{z-z_{0}}=\frac{1}{z-z_{0}} \int_{z_{0}}^{z} f^{\prime}(z) d z
$$

die Gültigkeit von $(30,2)$ vorausgesetzt,

$$
\operatorname{Lim}_{z \rightarrow z_{0}} \frac{f(z)-w_{0}}{z-z_{0}}=\frac{\gamma}{\gamma_{1}} \lambda
$$

Daher muss wegen (30, I) für $\gamma \neq \gamma_{1}$ entweder $\lambda=0$ oder $\lambda=\infty$ sein. Es wird sich übrigens später ergeben, dass $(30, \mathrm{I})$ und $(30,2)$ für $\gamma>\gamma_{1}$ stets mit $\lambda=0$ gelten und für $\gamma<\gamma_{1}$ stets mit $\lambda=\infty$. (Vgl. Satz XXI, Nr. 62.)

Über den Fall $\gamma=0$ vgl. Nr. 68 .

31. Verschärfung einer Ungleichung von Herrn S. Warschawski. Herr S. Warschawski hat vor einigen Jahren die folgende Tatsache gefunden ${ }^{25}$ :

Es sei $C$ eine geschlossene Jordankurve in der $w$-Ebene, die in einem Randpunkt $w_{0}$ eine Ecke der Öfnung $\pi \tau$, o $<\tau<2$, besitzt. $z=\varphi($ w $)$ bildet das Innere $G$ von $C$ auf $E_{z}$ konform $a b . \quad W$ bezeichne den von zwei von $w_{0}$ ausgehenden, symmetrisch aur Winkelhalbierenden der Ecke in $w_{0}$ verlaufenden Strahlen gebildeten Winkel der Öfnung $2 \psi, 0 \leqq \psi<\frac{\pi \tau}{2}$. Genügt dann $C$ in der Nähe von $w_{0}$ einer gewissen Bedingung - der Bedingung der "linearen Unbewalltheit" - so gibt es drei positive Konstanten $K, K^{\prime}, q$, unter denen $K, K^{\prime}$ von $\psi$ unabhängig sind, sowie zu jedem $w \neq w_{0}$ auf $C$ mit $\left|w-w_{0}\right| \leqq q$ zwei positive Zahlen $R, R^{\prime}$, die mit $w \rightarrow w_{0}$ gegen o konvergieren, so dass für alle Punkte $c$ in $W$ und $G$ mit $\left|c-w_{0}\right|=R$, und alle Punkte $c^{\prime}$ in $W$ und $G$ mit $\left|c^{\prime}-w_{0}\right|=R^{\prime}$ die Relation gilt:

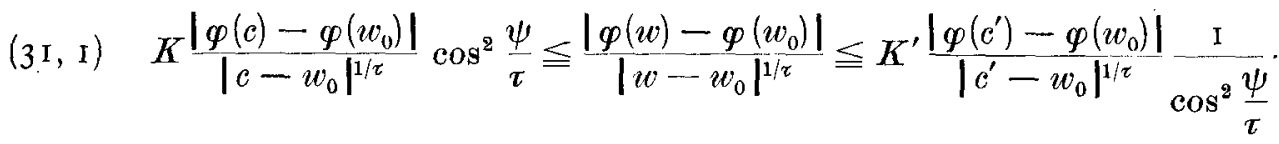

Mit Hilfe des Satzes VIII sieht man nun leicht ein, dass in dieser Ungleichung die Faktoren $\cos ^{2} \frac{\psi}{\tau}$ und $\frac{\mathrm{I}}{\cos ^{2} \frac{\psi}{\tau}}$ weggelassen werden können, wenn man

24 Vgl. VALIRON (2), p. 72; AHLFORS (1), pp. 28-29.

25 WARSCHAWSKI (1), pp. $36 \mathbf{1}, 369$. 
Über den Habitus der konformen Abbildung am Rande des Abbildungsbereiches. 129

die Konstanten $K, K^{\prime}$ mit gewissen Faktoren multipliziert, die gegen I konvergieren, wenn $c$ und $c^{\prime}$ gegen $v_{0}$ streben.

In der Tat ergibt sich, wenn $c=c_{0}$ und $c^{\prime}=c_{0}^{\prime}$ auf der Winkelhalbierenden des Eckenwinkels in $w_{0}$ angenommen werden, da dann ja $\psi=0$ gesetzt werden kann,

$(3 \mathrm{I}, \dot{2}) \quad K \frac{\left|\varphi\left(c_{0}\right)-\varphi\left(w_{0}\right)\right|}{\left|c_{0}-w_{0}\right|^{1 / \tau}} \leqq \frac{\left|\varphi(w)-\varphi\left(w_{0}\right)\right|}{\left|w-w_{0}\right|^{1 / \tau}} \leqq K^{\prime} \frac{\left|\varphi\left(c_{0}{ }^{\prime}\right)-\varphi\left(w_{0}\right)\right|}{\left|c_{0}^{\prime}-w_{0}\right|^{1 / \tau}}$

Andererseits folgt aus $(2 \mathrm{I}, 2)$

$$
\frac{\left|\varphi\left(c_{0}\right)-\varphi\left(w_{0}\right)\right|}{\left|c_{0}-w_{0}\right|^{1 / \tau}}=\frac{\left|\varphi(c)-\varphi\left(w_{0}\right)\right|}{\left|c-w_{0}\right|^{1 / \tau}}(\mathrm{I}+\varepsilon),
$$

und

$(3 \mathrm{I}, 4)$

$$
\frac{\left|\varphi\left(c_{0}^{\prime}\right)-\varphi\left(w_{0}\right)\right|}{\left|c_{0}^{\prime}-w_{0}\right|^{1 / \tau}}=\frac{\left|\varphi\left(c^{\prime}\right)-\varphi\left(w_{0}\right)\right|}{\left|c^{\prime}-w_{0}\right|^{1 / \tau}}\left(\mathrm{I}+\varepsilon^{\prime}\right)
$$

wo $\varepsilon^{\prime}$ und $\varepsilon$ gegen $\circ$ streben, wenn $c_{0}, c_{0}{ }^{\prime}$ gegen $w_{0}$ konvergieren. Setzt man nun $(3 I, 3)$ und $(3 I, 4)$ in $(3 I, 2)$ ein, so ergibt sich unsere Behauptung sofort. ${ }^{26}$

32. Asymptotisches Verhalten der höheren Ableitungen von $f(z)$. Unter den Voraussetzungen des Satzes VIII lässt sich aus den Relationen $(2 \mathrm{I}, 2)$ und $(2 \mathrm{I}, 3)$ eine interessante Folgerung ziehen. Wir setzen

$$
z_{1}=z, \quad z_{2}=z+t\left(z_{0}-z\right)
$$

so dass

$$
\frac{z_{2}-z_{0}}{z_{1}-z_{0}}=\mathrm{I}-t
$$

ist. Strebt nun $z$ gegen $z_{0}$ innerhalb eines Winkelraumes mit der Spitze in $z_{0}$, dessen Schenkel zur Winkelhalbierenden der inneren Ecke von $G_{1}$ in $z_{0}$ unter einem Winkel $\omega<\frac{\gamma_{1}}{2}$ geneigt sind, und schränkt man $t$ auf den Bereich

$$
|t| \leqq \frac{1}{2} \sin \frac{\mathrm{I}}{2}\left(\frac{\gamma_{1}}{2}-\omega\right)
$$

${ }^{26}$ Es ist übrigens klar, dass die Formel $(2 \mathrm{I}, 2)$ des Satzes VIII im zweiten Unterfall des Falles d) dieses Satzes eine wesentliche Verschärfung der Warschawskischen Ungleichungen liefert, wenn die Kurve $C$ in $w_{0}$ eine $L$-Tangente besitzt, bzw. wenn dies für die beiden in $w_{0}$ zusammenstossenden Bogen von $C$ zutrifft. - Wie ich von Hrn. Warschawski nach Mitteilung des obigen Resultats erfahre, ergibt es sich auch direkt aus einem neuen von ihm inzwischen gefundenen Beweis seines Satzes.

17-34472. Acta mathematica. 64. Imprimé le 31 octobre 1934. 
ein, so bleibt auch $z_{2}$ in einer Winkelumgebung von $z_{0}$, sobald $z$ nahe genug bei $z_{0}$ liegt. Zugleich sind dann die Bedingungen (2 I, I) erfüllt, so dass wegen $(2 \mathrm{I}, 2)$ und $(2 \mathrm{I}, 3)$

$$
\begin{gathered}
\frac{f\left(z+t\left(z_{0}-z\right)\right)-f\left(z_{0}\right)}{f(z)-f\left(z_{0}\right)} \rightarrow(\mathrm{I}-t)^{\frac{\gamma}{\gamma_{1}}} \\
\frac{f^{\prime}\left(z+t\left(z_{0}-z\right)\right)}{f^{\prime}(z)} \rightarrow(\mathrm{I}-t)^{\gamma_{1}}-1 .
\end{gathered}
$$

gilt, wenn $z$ im Winkel gegen $z_{0}$ strebt, und zwar gleichmässig in einer Umgebung von $t=0$, deren Radius allerdings von der Winkelumgebung von $z_{0}$ abhängig ist, innerhalb deren $z$ bleibt. Daher müssen in $(32, \mathrm{I})$ und $(32,2)$ auch die Koeffizienten der verschiedenen Potenzen von $t$ links gegen die entsprechenden Koeffizienten rechts konvergieren. Die Koeffizienten bei $t^{v}$ links und rechts sind aber resp.

$$
\begin{aligned}
& \frac{\left(z_{0}-z\right)^{v}}{\nu !} \frac{f^{(v)}(z)}{f(z)-f\left(z_{0}\right)}, \quad(-\mathrm{I})^{v}\left(\begin{array}{c}
\frac{\gamma}{\gamma_{1}} \\
v
\end{array}\right) \\
& \frac{\left(z_{0}-z\right)^{v}}{\nu !} \frac{f^{(v+1)}(z)}{f^{\prime}(z)}, \quad(-\mathrm{I})^{v}\left(\begin{array}{c}
\frac{\gamma}{\gamma_{1}}-\mathrm{I} \\
v
\end{array}\right),
\end{aligned}
$$

so dass sich schliesslich für $y>0$

$$
\begin{gathered}
\left(z-z_{0}\right)^{v} \frac{f^{(v)}(z)}{f(z)-f\left(z_{0}\right)} \rightarrow \frac{\gamma}{\gamma_{1}}\left(\frac{\gamma}{\gamma_{1}}-\mathrm{r}\right) \cdots\left(\frac{\gamma}{\gamma_{1}}-\nu+\mathrm{I}\right), \\
\left(z-z_{0}\right)^{\nu} \frac{f^{(\nu+1)}(z)}{f^{\prime}(z)} \rightarrow\left(\frac{\gamma}{\gamma_{1}}-\mathrm{I}\right)\left(\frac{\gamma}{\gamma_{1}}-2\right) \cdots\left(\frac{\gamma}{\gamma_{1}}-\nu\right)
\end{gathered}
$$

ergibt, wenn $z$ im Winkel gegen $z_{0}$ konvergiert. - Man übersieht übrigens sofort, dass die Formeln $(32,2),(32,4)$ aus $(32,1),(32,3)$ direkt vermöge der Formel ( I I, 3) des Randverzerrungssatzes folgen.

Dabei war bisher $\gamma>0$ vorausgesetzt. Wir werden aber in der $\mathrm{Nr} .64$ beweisen, dass die Relation $(21,2)$ auch dann richtig bleibt, wenn unter den Voraussetzungen des Satzes VIII $o=\gamma<\gamma_{1}$ ist. Daher gelten die Formeln $(32, \mathrm{I})$ und $(32,3)$ auch für $\gamma=0$. Was aber die Formel $(2 \mathrm{I}, 3)$ anbetrifft, so gilt sie, wie wir in der $\mathrm{Nr} .68$ beweisen werden, auch für $\gamma=0$, sofern die in $z_{0}$ und $w_{0}$ zusammenstossenden Randzweige von $G_{1}$ und $G$ dort $L$-Tangenten besitzen. Daher gelten die Formeln $(32,2),(32,4)$, sobald diese letzten Voraussetzungen erfüllt sind, anch für $o=\gamma<\gamma_{1}$. 


\section{Teil II. Ergänzungssätze zum Randverzerrungssatz.}

\section{§ 7. Erste Erweiterung des Satzes I.}

33. Formulierung des Satzes XII. Aus der Formel $(7,5)$ folgt sofort, dass, wenn die Funktion $\chi(\vartheta)$ des Satzes I auf einer $\vartheta_{0}$-Menge $M$ gleichmässig stetig ist, dann auch die Relation $(7,4)$ gleichmässig für alle Punkte von $\boldsymbol{M}$ gilt. Denn es ist klar, dass man dann für alle Punkte $\vartheta_{0}$ von $M$ mit einer gemeinsamen Funktion $\lambda(\boldsymbol{y})$ - mit einem gemeinsamen $»$ Lipschitzschen Modul» - auskommen kann. Anderseits ist es unerheblich, dass die Funktion $\chi(\vartheta)$ im ganzen Intervall $(-\pi, \pi)$ beschränkt ist, da es offenbar nur auf ihr Verhalten in der Nähe der Punkte von $M$ ankommt. Und ebenso wenig braucht die Darstellbarkeit von $f(z)$ in der Gestalt $(7,2)$ vorausgesetzt zu werden, wenn $\chi(\vartheta)$ als Randwert im Winkel von $\Re f(z)$ in der Umgebung der Punkte von $M$ vorausgesetzt wird.

Wir werden daher unsere Formulierung des Satzes I in folgender Weise erweitern :

Satz XII: Es sei $f(z)$ eine fïr $|z|<I$ reguläre analytische Funktion, und es sei $M$ eine solche Punktmenge auf $E_{z}$, dass $\mathfrak{R} f(z)$ für ein festes $\varepsilon>0$ in der $\varepsilon$-Umgebung jedes Punktes von $M$ innerhalb von $K_{z}$ gleichmässig beschränkt ist, in der $\varepsilon$-Umgebung eines jeden Punktes von $\boldsymbol{M}$ auf $E_{z}$ die Randwerte von $\Re f(z)$ bei $A n$ näherung im Winkel existieren, bis auf Punkte einer gewissen auf $E_{z}$ gelegenen Nullmenge, und eine in den Punkten von $M$ gleichmässig stetige Funktion bilden. Dann gilt für $f^{\prime}(z)$ bei allseitiger Annäherung aus $K_{z}$ gegen die Punkte von $M$ gleichmässig

$$
f^{\prime}(z)=o\left(\frac{\mathbf{I}}{\mathrm{I}-|z|}\right)
$$

Hier kann $M$ natürlich auch aus einem einzigen Punkt bestehen.

34. Beweis. In der Tat folgt aus den Voraussetzungen des Satzes XII, dass man auf $E_{z}$ endlich viele getrennte, etwa zyklisch geordnete abgeschlossene Kreisbogen $B_{1}, B_{2}, \ldots, B_{m}$ derart angeben kann, dass erstens die Menge $M$ samt ihren Häufungsstellen aus lauter innern Punkten der Kreisbogen $B_{\mu}$ besteht und dass zweitens $\Re f(z)$ in der Umgebung der abgeschlossenen Kreisbogen $B_{1}, \ldots, B_{m}$ innerhalb von $E_{z}$ gleichmässig beschränkt ist. Bedeutet nun allgemein $B_{u}^{*}$ den zwischen $B_{\mu}$ und $B_{\mu+1}$ bzw. $B_{m}$ und $B_{1}$ liegenden Kreisbogen von $E_{z}$, so lassen 
sich die Endpunkte von jedem $B_{\mu}^{*}$ durch einen bis auf seine Endpunkte ganz innerhalb von $E_{z}$ verlaufenden Kreisbogen $\alpha_{\mu}$ verbinden, auf dem $\mathfrak{R} f(z)$ gleichmässig beschränkt ist. Bildet man nun das Innere des von den Kreisbogen $B_{\mu}$ und $\alpha_{\mu}$ begrenzten Fläschenstückes konform auf das Innere des Einheitskreises in der $w$-Ebene $\mathrm{ab}$, so ist die Abbildungsfunktion $w=F(z)$ bekanntlich innerhalb der Kreisbogen $B_{\mu}$ regulär, und der absolute Betrag ihrer Ableitung ist in der Nähe der Punkte von $\boldsymbol{M}$ gleichmässig nach oben und unten durch positive Zahlen beschränkt. Daher geht $f(z)$ in eine Funktion $f_{1}(w)$ über, für die die Randfunktion von $\Re f_{1}(w)$ in der $w$-Ebene gleichmässig beschränkt und auf der ganzen Punktmenge $M_{1}$, die der Menge $M$ entspricht, gleichmässig stetig ist. Daher ist $f_{1}(w)$ in der $w$-Ebene evtl. nach Abzug einer geeigneten rein imaginären Konstanten durch das Poissonsche Integral vom Typus $(7,2)$ darstellbar, so dass nach Satz I die zu $(7,4),(7,5)$ analogen Relationen gleichmässig in allen Punkten von $M_{1}$ gelten. Nach dem über die Ableitung der Abbildungsfunktion Gesagten gilt aber dann, wegen $f^{\prime}(z)=f_{1}^{\prime}(w) F^{\prime}(z)$, auch die Relation $(7,4)$ bei allseitiger Annäherung an die Punkte von $M$ gleichmässig auf $M$, wie behauptet.

\section{§ 8. Zweite Erweiterung des Satzes I. (Durchschnittsstetigkeit).}

35. Asymptotische Abschätzungen von $I_{n}(z)$. Für das Folgende brauchen wir eine Abschätzung des Integrals

$(35, \mathrm{I}) \quad I_{n}(z)=\int_{0}^{\frac{\pi}{2}} \frac{\sin \vartheta d \vartheta}{\left(\mathrm{I}-2 r \cos (\vartheta-\varphi)+r^{2}\right)^{\frac{n+2}{2}}}, z=r e^{i \varphi}, \frac{\pi}{4}>\varphi>-\frac{\pi}{4}, n \geqq 0$.

Führen wir hier $\vartheta-\varphi=\vartheta_{1}$ als neue Integrationsvariable ein, so erhalten wir

$$
\begin{array}{r}
I_{n}(z)=\cos \varphi \int_{-\varphi}^{\frac{\pi}{2}-\varphi} \frac{\sin \vartheta d \vartheta}{\left(\mathrm{I}-2 r \cos \vartheta+r^{2}\right)^{\frac{n+2}{2}}}+\sin \varphi \int_{-\varphi}^{\frac{\pi}{2}-\phi} \frac{\cos \vartheta d \vartheta}{\left(\mathrm{r}-2 r \cos \vartheta+r^{2}\right)^{\frac{n+2}{2}}}= \\
=\cos \varphi J_{1}+\sin \varphi J_{2} .
\end{array}
$$

Hier lässt sich aber $J_{1}$ direkt ausintegrieren, und man erhält für $n>0$

$$
-\left.\frac{\mathrm{I}}{r \cdot n} \frac{\mathrm{I}}{\left(\mathrm{I}-2 r \cos \vartheta+r^{2}\right)^{\frac{n}{2}}}\right|_{-\varphi} ^{\frac{\pi}{2}-\varphi}=\frac{\mathrm{I}}{r n} \frac{\mathrm{I}}{|\mathrm{I}-z|^{n}}-\frac{\mathrm{I}}{r n} \frac{\mathrm{I}}{\left(\mathrm{I}-2 r \sin \varphi+r^{2}\right)^{\frac{n}{2}}} .
$$


Über den Habitus der konformen Abbildung am Rande des Abbildungsbereiches. 133 Für $n=\mathrm{o}$ ergibt sich analog

$$
\left.\frac{\mathrm{I}}{2 r} \lg \left(\mathrm{I}-2 r \cos \vartheta+r^{2}\right)\right|_{-\varphi} ^{\frac{\pi}{2}-\varphi}
$$

Zusammenfassend folgt:

$(35,2) \quad J_{1}=\frac{\mathrm{I}+\varepsilon_{1}^{(n)}(z)}{n} \frac{\mathrm{I}}{|\mathrm{I}-z|^{n}}, n>0 ; \quad J_{1}=\left(\mathrm{I}+\varepsilon_{1}(z)\right) \lg \frac{\mathrm{I}}{|\mathrm{I}-z|}, n=0$,

wo $\varepsilon_{1}^{(n)}, \varepsilon_{1}(z)$, ebenso wie später $\varepsilon_{2}(z), \varepsilon_{3}(z), \ldots$ gegen Null konvergieren, wenn $z$ gegen I strebt. Für das Integral $J_{2}$ erhalten wir

$(35,3) \quad J_{2}=\int_{-\varphi}^{\frac{\pi}{2}-\varphi} \frac{\cos \frac{\vartheta}{2} d \vartheta}{\left(\mathrm{I}-2 r \cos \vartheta+r^{2}\right)^{\frac{n+2}{2}}}+O\left(\int_{-\varphi}^{\frac{\pi}{2}-\varphi} \frac{\cos \frac{\vartheta}{2} \sin ^{2} \frac{\vartheta}{2} d \vartheta}{\left(\mathrm{I}-2 r \cos \vartheta+r^{2}\right)^{\frac{n+2}{2}}}\right)$,

da, wie man leicht nachprüft, für $-\frac{\pi}{4} \leqq \vartheta \leqq \frac{3 \pi}{4}$

$$
\cos \vartheta-\cos \frac{\vartheta}{2}=O\left(\sin ^{2} \frac{\vartheta}{2}\right)=O\left(\cos \frac{\vartheta}{2} \sin ^{2} \frac{\vartheta}{2}\right)
$$

ist. Die Integrale rechts in $(35,3)$ aber gehen, $\sin \frac{\vartheta}{2}=\gamma$, I $-r=\varrho$ gesetzt, über in

$$
2 \int_{-\sin \frac{\varphi}{2}}^{\cos \left(\frac{\varphi}{2}+\frac{\pi}{4}\right)} \frac{d \gamma}{\left(\sqrt{\varrho^{2}+4 r \gamma^{2}}\right)^{n+2}} b z w \cdot 2 \int_{-\sin \frac{\varphi}{2}}^{\cos \left(\frac{\varphi}{2}+\frac{\pi}{4}\right)} \frac{\gamma^{2} d \gamma}{\left(\sqrt{\varrho^{2}+4 r \gamma^{2}}\right)^{n+2}} .
$$

Setzt man aber hier $2 \sqrt{r} \gamma=\varrho x$, so verwandeln sich diese Integrale in

$$
\begin{aligned}
& \frac{\mathrm{I}}{\sqrt{r} \varrho^{n+1}} \int_{\left(\mathrm{I}+x^{2}\right)^{\frac{n+2}{2}}}^{\frac{2 \sqrt{r} \cos \left(\frac{\varphi}{2}+\frac{\pi}{4}\right)}{d}} \text { bzw. } \frac{d x}{4 r^{\frac{3}{2}} \varrho^{n-1}} \int_{\left(\mathrm{I}+x^{2}\right)^{\frac{n+2}{2}}}^{\varrho} \frac{x^{2} d x}{\left(\sqrt{r} \cos \left(\frac{\varphi}{2}+\frac{\pi}{4}\right)\right.} \\
& -\frac{2 \sqrt{r} \sin \frac{\varphi}{2}}{\rho} . \quad-\frac{2 \sqrt{r} \sin \frac{\varphi}{2}}{\rho}
\end{aligned}
$$


so dass für $n \geqq 0$

$(35,4) \quad J_{2}=\frac{\mathrm{I}+\varepsilon_{2}(z)}{\rho^{n+1}}\left(\int_{0}^{\infty} \frac{d x}{\left(1+x^{2}\right)^{\frac{n+2}{2}}}+\operatorname{sgn} \varphi \int_{0}^{\frac{2 \sqrt{r} \sin \frac{|\varphi|}{2}}{e^{-\frac{\mid \varphi}{2}}}} \frac{d x}{\left(\mathrm{I}+x^{2}\right)^{\frac{n+2}{2}}}\right)+O\left(\frac{\mathrm{I}}{\varrho^{n-1}} \int_{1}^{\frac{3}{\rho}} \frac{d x}{x^{n}}\right)$

ist.

Ist nun, wie im Satz III (Nr. 10), $\psi=-\arg (\mathrm{I}-z), \frac{\pi}{2}>\psi>-\frac{\pi}{2}$, so ergibt sich unter Benutzung der Formel (10,5)

$$
\begin{gathered}
\begin{aligned}
(35,5) \quad \varrho^{n} I_{n}(z)=\frac{\cos ^{n} \psi}{n}+\frac{\left(\mathrm{I}+\varepsilon_{3}(z)\right) \varphi}{\varrho}\left(\int_{0}^{\infty} \frac{d x}{\left(\mathrm{I}+x^{2}\right)^{\frac{n+2}{2}}}+\operatorname{sgn} \varphi \int_{0}^{\frac{\rho}{2 \sqrt{r} \sin \frac{|\varphi|}{2}}} \frac{d x}{\left(\mathrm{I}+x^{2}\right)^{\frac{n+2}{2}}}\right)+ \\
+\varepsilon_{4}(z), n>0 .^{27}
\end{aligned} \\
(35,6) \quad I_{0}(z)=\left(\mathrm{I}+\varepsilon_{5}(z)\right) \lg _{|\mathrm{I}-z|}+\frac{\varphi}{\varrho}\left(\mathrm{I}+\varepsilon_{6}(z)\right)\left(\frac{\pi}{2}+\operatorname{arctg}\left(\frac{2 \sqrt{r} \sin \frac{\varphi}{2}}{\varrho}\right)\right)
\end{gathered}
$$

In $(35,5)$ konvergiert der erste Summand rechts sicher gegen Null, falls mit $z \rightarrow 1$ zugleich $|\psi|$ gegen $\frac{x}{2}$ strebt. Sein $\overline{\operatorname{Lim}}$ ist aber auf jeden Fall $\leqq \frac{\mathrm{I}}{n}$. Der zweite Summand in $(35,5)$ und $(35,6)$ verhält sich aber verschieden, je nachdem $\varphi$ - und damit auch $\psi-$ positiv oder negativ ist. Ist $\varphi$ positiv, so liegt der Wert der Klammer zwischen

$$
\int_{0}^{\infty} \frac{d x}{\left(1+x^{2}\right)^{\frac{n+2}{2}}} \text { und } 2 \int_{0}^{\infty} \frac{d x}{\left(1+x^{2}\right)^{\frac{n+2}{2}}}, \quad n \geqq 0 .
$$

Bleibt dann $|\psi|$ kleiner als eine feste Zahl $<\frac{\pi}{2}$ - dies läuft darauf hinaus, dass

$$
\begin{aligned}
& 27 \text { Die Werte des Integrals } \int_{0}^{\infty} \frac{d x}{\left(\mathrm{I}+x^{2}\right)^{\frac{n+2}{2}}} \text { sind bekanntlich } \\
& \left(\mathrm{I}-\frac{\mathrm{I}}{n}\right)\left(\mathrm{I}-\frac{\mathrm{I}}{n-2}\right) \ldots\left(\mathrm{I}-\frac{\mathrm{I}}{2}\right)^{\frac{\pi}{2}} \text { bzw. }\left(\mathrm{I}-\frac{\mathrm{I}}{n}\right)\left(\mathrm{I}-\frac{\mathrm{I}}{n-2}\right) \ldots\left(\mathrm{I}-\frac{\mathrm{I}}{3}\right),
\end{aligned}
$$

je nachdem ob $n$ gerade oder ungerade $>\mathrm{I}$ ist. Für $n=\mathrm{I}$ ist sein Wert $=\mathrm{I}$. 
Über den Habitus der konformen Abbildung am Rande des Abbildungsbereiches. 135 $z$ im Winkel gegen I strebt - so ist dann, wegen der ersten Formel (1o, 7), wenn dort $\vartheta$ durch $\varphi$ ersetzt wird, $\frac{\varphi}{\varrho}=\operatorname{tg} \psi+O(\varphi)$, so dass $(35,7) \quad \varrho^{n} I_{n}(z)=\frac{\cos ^{n} \psi}{n}+\operatorname{tg} \psi \int_{-\psi}^{\frac{\pi}{2}} \cos ^{n} x d x+\varepsilon_{7}(z), \quad|\psi| \leqq \frac{\pi}{2}-\delta, \delta>0, n>0$ $(35,8)$

$$
I_{0}(z)=\left(\mathrm{I}+\varepsilon_{8}(z)\right) \lg \frac{\mathrm{I}}{|\mathrm{I}-z|}, \quad|\psi| \leqq \frac{\pi}{2}-\delta, \delta>0
$$

wird. ${ }^{28}$ Und die gleichen Formeln gelten auch für negative $\varphi$, solange $z$ gegen I im Winkel strebt.

Wir erhalten daher insbesondere für $z \rightarrow \mathrm{I}, n>0,|\psi| \leqq \frac{\pi}{2}-\delta, \delta>0$ :

$$
\varrho^{n} I_{n}(z) \leqq \gamma_{n}(\delta)+\varepsilon_{7}(z)
$$

$$
\gamma_{n}(\delta) \leqq \frac{\mathrm{I}}{n}+\operatorname{ctg} \delta \int_{-\frac{\pi}{2}+\delta}^{\frac{\pi}{2}} \cos ^{n} x d x
$$

Nähert sich aber $\psi$ dem Werte $\frac{\pi}{2}$, so nimmt der Faktor $\frac{\varphi}{\varrho}$ und mit ihm die rechten Seiten von $(35,7)$ und $(35,90)$ beliebig grosse Werte an.

Ist dagegen $\varphi$ negativ und kommt dann $\psi$ dem Werte $-\frac{\pi}{2}$ beliebig nahe, so sind die Integrale in den Klammern rechter Hand in $(35,5)$ und $(35,6)$ $o\left(\left(\frac{\varrho}{\varphi}\right)^{n+1}\right)$, so dass sich wegen (Io, 7) ergibt:

$$
\begin{aligned}
& \rho^{n} I_{n}(z)=O\left(\cos ^{n} \psi\right)+\varepsilon_{8}(z), \quad-\frac{\pi}{4}>\psi>\frac{\pi}{2}, n>0, \\
& I_{0}(z)=\left(\mathrm{I}+\varepsilon_{9}(z)\right) \lg _{|\mathrm{I}-z|}, \quad \frac{\mathrm{I}}{2}-\delta>\psi>-\frac{\pi}{2} .
\end{aligned}
$$

28 Das Integral $\int_{-\operatorname{tg} \psi}^{\infty} \frac{d x}{\left(1+x^{2}\right)^{\frac{n+2}{2}}}$ geht nämlich dureh die Substitution $x=\operatorname{tg} y$ in das Integral $\int_{-\psi}^{\frac{\pi}{2}} \cos ^{n} y d y$ über. 
Für das Integral aber

$$
\int_{-\frac{\pi}{2}}^{0} \frac{|\sin \vartheta| d-2}{\left(1-2 r \cos (\vartheta-\varphi)+r^{2}\right)^{\frac{n+2}{2}}}=I_{n}(\bar{z})
$$

gelten offenbar die gleichen Abschätzungen $(35,9)-(35,92)$, wenn in ihnen $\varphi$ und $\psi$ durch $-\varphi,-\psi$ ersetzt werden.

36. Punkte der Durchschnittsstetigkeit von $\chi(\vartheta)$. Satz XIII. Es sei $\chi(\vartheta)$ eine im Intervall $(-\pi, \pi)$ nach LeBrsaue integrierbare Funktion, für die die Relation gilt

$$
\frac{\mathrm{I}}{\vartheta-\vartheta_{0}} \int_{\vartheta_{0}}^{\vartheta} \chi(\vartheta) d \vartheta \rightarrow \chi\left(\vartheta_{0}\right), \quad \vartheta \rightarrow \vartheta_{0}
$$

Bildet man mit Hilfe des Poissonschen Integrals die Funktion

$$
f(z)=\frac{1}{2 \pi} \int_{-\pi}^{\pi} \frac{e^{i \vartheta}+z}{e^{i \vartheta}-z} \chi(\vartheta) d \vartheta
$$

so gilt fiir ihre n-te Ableitung, $n \geqq \mathrm{I}$,

$$
f^{(n)}(z)=\frac{n !}{\pi} \int_{-\pi}^{\pi} \frac{e^{i \vartheta} \chi(\vartheta) d \vartheta}{\left(e^{i \vartheta}-z\right)^{n+1}}
$$

wenn $z$ aus dem Innern von $E_{z}$ im Winkel gegen $z_{0}=e^{i \vartheta_{0}}$ strebt:

$$
\left(z-z_{0}\right)^{n} f^{(n)}(z) \rightarrow 0
$$

Die Behauptung gilt gleichmässig für eine Punktmenge $M$ von Punkten $e^{i 9_{0}}$ auf $E_{z}$, für die $\chi(\vartheta)$ gleichmässig beschränkt ist und die Relation $(36$, I) gleichmässig gilt. Dabei ist natürlich zu verlangen, dass die Winkel, innerhalb deren z jeweils gegen $e^{i \vartheta_{0}}$ strebt, gleiche Oeffnungen haben und ähnlich in bezug auf die entsprechenden Radien liegen. ${ }^{29}$

37. Beweis. Nach Voraussetzung ist das Integral $\int_{-\pi}^{\pi}|\chi(\vartheta)| d \vartheta$ beschränkt,

${ }^{29}$ Für $n=\mathrm{I}$ findet $\operatorname{sich}(36,4)$ in Lichtenstern (2), p. 20. 
Über den Habitus der konformen Abbildung am Rande des Abbildungsbereiches. 137 und wir können offenbar $\int_{-\pi}^{\pi}|\chi(\vartheta)| d \vartheta \leqq \frac{\mathrm{I}}{2}$ annehmen. Ebenso können wir annehmen, dass $|\chi(\vartheta)| \leqq \frac{I}{2}$ auf der ganzen Menge $M$ gilt. Wir denken uns nun die Funktion $\chi(\vartheta)$ über die ganze Zahlengerade fortgesetzt auf Grund der Forderung der Periodizität mit der Periode $2 \pi$ und bilden die Funktion

$$
\Phi_{\vartheta_{0}}(\vartheta)=\int_{0}^{\vartheta}\left[\chi\left(\vartheta+\vartheta_{0}\right)-\chi\left(\vartheta_{0}\right)\right] d \vartheta, \quad-\pi<\vartheta \leqq \pi
$$

Dann liefert die partielle Integration

$$
\begin{aligned}
(37,2) \quad f(z)-\chi\left(\vartheta_{0}\right)=\frac{\mathrm{I}}{2 \pi} \int_{-\pi}^{\pi} \frac{e^{i\left(\vartheta+\vartheta_{0}\right)}+z}{e^{i\left(\vartheta+\vartheta_{0}\right)}-z}\left[\chi\left(\vartheta+\vartheta_{0}\right)-\chi\left(\vartheta_{0}\right)\right] d \vartheta= \\
\quad=\frac{i z}{\pi} \int_{-\pi}^{\pi} \frac{e^{i\left(\vartheta+\vartheta_{0}\right)}}{\left(e^{i\left(\vartheta+\vartheta_{0}\right)}-z\right)^{2}} \Phi_{\vartheta_{0}}(\vartheta) d \vartheta+\left.\frac{\mathrm{I}}{2 \pi} \frac{e^{i\left(\vartheta+\vartheta_{0}\right)}+z}{e^{i\left(\vartheta+\vartheta_{0}\right)}-z} \Phi_{\vartheta_{0}}(\vartheta)\right|_{-\pi} ^{\pi}
\end{aligned}
$$

Hier ist aber das letzte Glied auf der rechten Seite gleich

$$
\begin{aligned}
& \frac{\mathrm{I}}{2 \pi} \frac{e^{i\left(\pi+\vartheta_{0}\right)}+z}{e^{i\left(\pi+\vartheta_{0}\right)}-z}\left(\boldsymbol{\Phi}_{\vartheta_{0}}(\pi)-\boldsymbol{\Phi}_{\vartheta_{0}}(-\pi)\right)= \frac{\mathrm{I}}{2 \pi} \frac{e^{i \vartheta_{0}}-z}{e^{i \vartheta_{0}}+z} \int_{-\pi}^{\pi}\left(\chi\left(\vartheta+\vartheta_{0}\right)-\chi\left(\vartheta_{0}\right)\right) d \vartheta= \\
&=\left(\frac{2 z_{0}}{z+z_{0}}-\mathrm{I}\right) x, \chi=\frac{1}{2 \pi} \int_{-\pi}^{\pi} \chi(\vartheta) d \vartheta-\chi\left(\vartheta_{0}\right) .
\end{aligned}
$$

Daher ergibt sich durch $n$-malige Differentiation von $(37,2)$

$$
\begin{aligned}
(37,3) \quad f^{(n)}(z)=\frac{(n+\mathrm{I}) ! i z}{\pi} \int_{-\pi}^{\pi} \frac{e^{i \vartheta} z_{0}}{\left(e^{i \vartheta} z_{0}-z\right)^{n+2}} \Phi_{\vartheta_{0}}(\vartheta) d \vartheta+ \\
\quad+\frac{n ! n i}{\pi} \int_{-\pi}^{\pi} \frac{e^{i \vartheta} z_{0}}{\left(e^{i \vartheta} z_{0}-z\right)^{n+1}} \Phi_{\vartheta_{0}}(\vartheta) d \vartheta+(-\mathrm{I})^{n} 2 z_{0} n ! x \frac{\mathrm{I}}{\left(z+z_{0}\right)^{n+1}} .
\end{aligned}
$$

18-34472. Acta mathematica. 64. Imprimé le 31 octobre 1934. 
Es genügt daher zu beweisen, dass aus $\Phi_{9_{0}}(\vartheta)=o(\vartheta)$ für $\vartheta \rightarrow 0$

$$
(\mathrm{I}-z)^{n} \int_{-\pi}^{\pi} \frac{e^{i \vartheta}}{\left(e^{i g}-z\right)^{n+2}} \Phi_{9_{0}}(\vartheta) d \vartheta=o(\mathrm{I})
$$

folgt, wenn $z$ im Winkel gegen I strebt. Setzt man aber $\boldsymbol{\Phi}_{\vartheta_{0}}(\vartheta)=\mathscr{\vartheta} \Psi(\vartheta)$, so folgt für $z=r e^{i \varphi},-\frac{\pi}{2}<\varphi<\frac{\pi}{2}$ :

$$
\left|\int_{-\pi}^{\pi} \frac{e^{i \vartheta} \Phi_{\vartheta_{0}}(\vartheta) d \vartheta}{\left(e^{i \vartheta}-z\right)^{n+2}}\right| \leqq \int_{-\pi}^{\pi} \frac{|\vartheta||\Psi(\vartheta)| d \vartheta}{\left(r^{2}-2 r \cos (\vartheta-\varphi)+1\right)^{\frac{n+2}{2}}} .
$$

Ist nun $|\Psi(\vartheta)| \leqq \varepsilon$ für $|\vartheta| \leqq d, \frac{\pi}{2}>d>0$, und beachten wir, dass für alle $\vartheta$ nach der Annahme $\left|\Phi_{\vartheta_{0}}(\vartheta)\right| \leqq I$ ist, so folgt

$$
\begin{aligned}
(37,4) & \left|\int_{-\pi}^{\pi} \frac{\vartheta \Psi(\vartheta) d \vartheta}{\left(r^{2}-2 r \cos (\vartheta-\varphi)+1\right)^{\frac{n+2}{2}}}\right| \leqq 2 \int_{d}^{\pi} \frac{d \vartheta}{\left(r^{2}-2 r \cos (\vartheta-\varphi)+1\right)^{\frac{n+2}{2}}+} \\
& +2 \varepsilon \int_{0}^{d} \frac{\vartheta d \vartheta}{\left(r^{2}-2 r \cos (\vartheta-\varphi)+1\right)^{\frac{n+2}{2}}} .
\end{aligned}
$$

Da aber für $0<\vartheta<\frac{\pi}{2}$ stets $\vartheta<\frac{\pi}{2} \sin \vartheta$ ist und das erste Integral rechter Hand in $(37,4)$ für $z \rightarrow \mathrm{I}$ gegen eine endliche, nur von $d$ abhängige Konstante strebt, folgt weiter wegen $(35,9)$

$$
\overline{\operatorname{Lim}}(\mathrm{I}-r)^{n}\left|\int_{-\pi}^{\pi} \frac{\vartheta \Psi(\vartheta) d \vartheta}{\left(r^{2}-2 r \cos (\vartheta-\varphi)+\mathrm{I}\right)^{\frac{n+2}{2}}}\right| \leqq x \varepsilon \gamma_{n}(\delta)
$$

wenn $z$ derart im Winkel gegen I strebt, dass $|\psi|=|\arg (1-z)| \leqq \frac{\pi}{2}-\delta$ bleibt. Und da hier $\varepsilon$ beliebig klein angenommen werden kann, folgt wegen $(35,90)$ die Behauptung des Satzes.

38. Die Schwankung von $f(z)$. Bildet man unter den Voraussetzungen von Satz XIII die Funktion $(36,2)$ für $|z|<1$, so konvergiert $\Re f(z)$ nach Fatou, wenn $z$ im Winkel gegen $e^{i 9_{0}}$ strebt, gegen $\chi\left(\vartheta_{0}\right)$. Dagegen braucht dann be- 
Über den Habitus der konformen Abbildung am Rande des Abbildungsbereiches. 139 kanntlich $\Im f(z)$ keinem Grenzwert zuzustreben; es sind vielmehr für die Existenz des Grenzwertes von $\mathfrak{J} f(z)$ weitere Zusatzbedingungen nötig. Wir wollen nun eine Aussage beweisen, die bereits unter den Voraussetzungen von Satz XIII für $\Im f(z)$ oder, was auf das Gleiche hinausläuft, für $f(z)$ selbst gilt. Sie liefert Aufschluss über die Schwankung der Werte von $f(z)$ in der Nähe von $e^{i \vartheta_{0}}$.

Satz XIV: Unter den Voraussetzungen von Satz XIII gilt, wenn $z_{1}$, $z_{2}$ im Winkel gegen $z_{0}=e^{i g_{0}}$ streben und zugleich $\left|\frac{z_{0}-z_{2}}{z_{0}-z_{1}}\right|$ zwischen beliebigen aber festen positiven Schranken bleibt,

$$
f\left(z_{1}\right)-f\left(z_{2}\right) \rightarrow \mathrm{o}
$$

und die Relation $(38, \mathrm{I})$ gilt ebenso wie der Satz XIII gleichmässig für eine Menge $M$ der Punkte $\vartheta_{0}$, für die die Voraussetzungen über $\chi(\vartheta)$ gleichmässig gelten.

Beweis: Es sei $\varepsilon$ eine beliebige positive Zahl. Sind dann die Punkte $z_{1}, z_{2}$ bereits so nahe bei $z_{0}$, dass für jeden Punkt $z$. der Strecke $z_{1} z_{2}$ die Relation

$$
\left|f^{\prime}(z)\right| \leqq \frac{\varepsilon}{\mathrm{I}-|z|}
$$

gilt, so folgt

$$
\left|f\left(z_{2}\right)-f\left(z_{1}\right)\right|=\left|\int_{z_{1}}^{z_{2}} f^{\prime}(z) d z\right| \leqq \varepsilon \frac{\left|z_{2}-z_{1}\right|}{m}
$$

wo $m$ der kleinste Wert von I $-|z|$ für einen der Punkte der Strecke $z_{1} z_{2}$ ist. Nun wird aber für eine innerhalb von $E_{z}$ gelegene Strecke die kürzeste Distanz von $E_{z}$ stets für einen der Endpunkte der Strecke erreicht, da eine Strecke ja stets innerhalb eines Kreises liegt, in dem ihre beiden Endpunkte liegen. Ist daher etwa $m=\mathrm{I}-\left|z_{1}\right|$, so folgt aus $(38,3)$ weiter

$$
\begin{aligned}
\left|f\left(z_{2}\right)-f\left(z_{1}\right)\right| \leqq \varepsilon \frac{\left|z_{0}-z_{1}\right|+\left|z_{0}-z_{2}\right|}{1-\left|z_{1}\right|} \leqq \varepsilon & \frac{\left|z_{0}-z_{2}\right|+\left|z_{0}-z_{1}\right|}{\left|z_{0}-z_{1}\right|} \leqq \\
& \leqq \varepsilon C\left(1+\frac{\left|z_{0}-z_{2}\right|}{\left|z_{0}-z_{1}\right|}\right)
\end{aligned}
$$

wo die Konstante $C$ allein vom Winkel abhängt, innerhalb dessen $z_{1}$ und $z_{2}$ gegen $z_{0}$ streben. Nach Voraussetzung über den Quotienten $\frac{\left|z_{0}-z_{2}\right|}{\left|z_{0}-z_{1}\right|}$ aber folgt hieraus die Behauptung, da $\varepsilon$ beliebig klein gewäht werden kann. 
Aus dem Satz XIV folgt leicht der bekannte Satz von Pringsheim, dass, wenn die Fourierreihe von $\chi(\vartheta)$ in $\vartheta=\vartheta_{0}$ konvergiert, die zu $\chi(\vartheta)$ konjugierte Funktion in $\vartheta=\vartheta_{0}$ keine einfache Sprungsingularität haben kann. Sonst würde nämlich $f(z)$ längs jeder von $e^{i 9_{0}}$ ausgehenden Sehne einen Grenzwert haben, der sich linear mit dem Sehnenwinkel änderte, was dem Satz XIV widerspricht. - Zu einer analogen Fragestellung vgl. J. Wouff, (3).

\section{§ 9. Dritte Erweiterung des Satzes I (Durchschnittsendlichkeit).}

39. Abschätzung von $f^{(n)}(z)$ für einen speziellen Fall der. Durchschnittsendlichkeit. Wir wollen nun die Funktion

$$
f(z)=\frac{\mathbf{I}}{2 \pi} \int_{-\pi}^{\pi} \frac{e^{i \vartheta}+z}{e^{i \vartheta}-z} \chi(\vartheta) d \vartheta
$$

und ihre Ableitungen für $z \rightarrow \mathrm{I}$ unter der Voraussetzung abschätzen, dass $\chi(\boldsymbol{9})$ zwischen $-\pi$ und $\pi$ nach Lebesgue integrabel ist und, $\int_{0}^{9} \chi(\vartheta) d \vartheta=\Phi(\vartheta)$ gesetzt,

$$
\begin{array}{ll}
\overline{\operatorname{Lim}} \frac{\Phi(\vartheta)}{\vartheta}= \pm k_{+}, & k_{+} \geqq 0, \text { für } \vartheta \downarrow o, \\
\overline{\operatorname{Lim}} \frac{\Phi(\vartheta)}{\vartheta}= \pm k_{-}, & k_{-} \geqq 0, \text { für } \vartheta \uparrow o
\end{array}
$$

ist, d. h. also unter der Voraussetzung, dass die vier derivierten Zahlen von $\boldsymbol{\Phi}(\vartheta)$ an der Stelle $\vartheta=0$ die Werte $\pm k_{+}$für die rechtsseitigen bzw. $\pm k-$ für die linksseitigen derivierten Zahlen haben.

Man beachte zuerst, dass, wenn wir die Integrationsgrenzen in $(39,1)$ durch $-\frac{\pi}{2}$ bzw. $\frac{\pi}{2}$ ersetzen, $f(z)$ sich um einen an der Stelle $z=\mathrm{I}$ regulären Summanden ändert, so dass alle Ableitungen dieses Summanden für $z \rightarrow \mathrm{I}$ sich endlichen Grenzwerten nähern. Wir dürfen daher für die hier herzuleitenden Abschätzungen annehmen, dass $\chi(\vartheta)$ ausserhalb des Intervalls $\left\langle-\frac{\pi}{2}, \frac{\pi}{2}\right\rangle$ verschwindet. Dann ergibt sich für $f(z)$ und $f^{(n)}(z)$, ähnlich wie beim Beweise des Satzes XIII, durch partielle Integration und die darauf folgende Differentiation, wenn wir zugleich $\Phi(\vartheta)=\sin \vartheta \Psi(\vartheta)$ setzen: 
Über den Habitus der konformen Abbildung am Rande des Abbildungsbereiches.

$(39,4) \quad f(z)=\frac{i z}{\pi} \int_{-\frac{\pi}{2}}^{\frac{\pi}{2}} \frac{e^{i \vartheta} \sin \vartheta \Psi(\vartheta) d \vartheta}{\left(e^{i \vartheta}-z\right)^{2}}+\frac{\mathrm{I}}{2 \pi}\left(\frac{i+z}{i-z} \Phi\left(\frac{\pi}{2}\right)-\frac{i-z}{i+z} \Phi\left(-\frac{\pi}{2}\right)\right)$,

$(39,5) \quad \frac{\pi}{(n+\mathrm{I}) !} f^{(n)}(z)=i z \int_{-\frac{\pi}{2}}^{\frac{\pi}{2}} \frac{e^{i \vartheta} \sin \vartheta \Psi(\vartheta) d \vartheta}{\left(e^{i 9}-z\right)^{n+2}}+\frac{n i}{n+\mathrm{I}} \int_{-\frac{\pi}{2}}^{\frac{\pi}{2}} \frac{e^{i \vartheta} \sin \boldsymbol{\vartheta} \Psi(\vartheta) d \vartheta}{\left(e^{i \vartheta}-z\right)^{n+1}}+$

$$
+\frac{i}{n+\mathrm{I}}\left(\frac{\Phi\left(\frac{\pi}{2}\right)}{(i-z)^{n+1}}-(-\mathrm{I})^{n} \frac{\Phi\left(-\frac{\pi}{2}\right)}{(\overrightarrow{i+z})^{n+1}}\right) \text {. }
$$

Hier sind nach Voraussetzung für 9 $10 \mathrm{bzw}$. 9 von $\Psi(\vartheta)$ gleich $\pm k_{+}$bzw. $\pm k_{-}$. Es sei nun $C$ eine obere Schranke von $|\Psi(\vartheta)|$ in $\left\langle-\frac{\pi}{2}, \frac{\pi}{2}\right\rangle$. Ist $\varepsilon$ eine beliebig kleine positive Zahl, so lässt sich dazu nach Voraussetzung ein solches $\delta>0$ finden, dass $|\Psi(9)| \leqq k_{+}+\varepsilon$ für $0<\boldsymbol{\vartheta} \leqq \delta$ und $|\Psi(\vartheta)| \leqq k_{-}+\varepsilon$ für $-\delta \leqq \vartheta<0$ ist. Dann ergibt sich aus $(39,4), z=r e^{i \varphi}$, $|\varphi|<\frac{\pi}{4}$ gesetzt, wegen $|z \pm i|>\sqrt{\frac{\mathrm{I}}{2}}>\frac{\mathrm{I}}{2}$ :

$$
\frac{\pi}{r}|f(z)| \leqq \int_{-\frac{\pi}{2}}^{\frac{\pi}{2}} \frac{|\sin \vartheta||\Psi(\vartheta)| d \vartheta}{\left|e^{i \vartheta}-z\right|^{2}}+\frac{4 C}{r}=\int_{-\frac{\pi}{2}}^{-\delta}+\int_{-\delta}^{0}+\int_{0}^{\delta}+\int_{\delta}^{\frac{\pi}{2}}+\frac{4 C}{r}
$$

$\frac{\pi}{r}|f(z)| \leqq C\left(\int_{\delta}^{\frac{\pi}{2}}+\int_{-\frac{\pi}{2}}^{-\delta^{\delta}} \frac{|\sin \vartheta| d \vartheta}{\left|e^{i \vartheta}-z\right|^{2}}+\left(k_{+}+\varepsilon\right) \int_{0}^{\delta} \frac{\sin \vartheta d \vartheta}{\left|e^{i \vartheta}-z\right|^{2}}+\left(k_{-}+\varepsilon\right) \int_{-\delta}^{0} \frac{|\sin \vartheta| d \vartheta}{\left|e^{i \vartheta}-z\right|^{2}}+\frac{4 C}{r}\right.$

oder endlich

$$
\left.\frac{\pi}{r}\left|f^{\prime}(z)\right|<C\left(\int_{\delta}^{\frac{\pi}{2}}+\int_{-\frac{\pi}{2}}^{-\delta}\right) \frac{|\sin \vartheta| d \vartheta}{\left|e^{i \vartheta}-z\right|^{2}}+\left(k_{+}+\varepsilon\right) I_{0}(z)+\left(k_{-}+\varepsilon\right)\right] I_{0}(\bar{z})+\frac{4 C}{r} .
$$

Genau ebenso erhalten wir aus $(39,5)$ 


$$
\begin{aligned}
\frac{\pi}{(n+\mathrm{I}) !}\left|f^{(n)}(z)\right| \leqq & C r\left(\int_{\delta}^{\frac{\pi}{2}}+\int_{-\frac{\pi}{2}}^{-\delta} \frac{|\sin \vartheta| d \vartheta}{\left|e^{i \vartheta}-z\right|^{n+2}}+C\left(\int_{\delta}^{\frac{\pi}{2}}+\int_{-\frac{\pi}{2}}^{-\delta} \frac{|\sin \vartheta| d \vartheta}{\left|e^{i \vartheta}-z\right|^{n+1}}+\right.\right. \\
& +\left(k_{+}+\varepsilon\right)\left(I_{n}(z)+I_{n-1}(z)\right)+\left(k_{-}+\varepsilon\right)\left(I_{n}(\bar{z})+I_{n-1}(\bar{z})\right)+\frac{2^{n+2} C}{n+\mathrm{I}} .
\end{aligned}
$$

Benutzen wir nunmehr die in Nr. 35 hergeleitete Abschätzung $(35,8)$ für $I_{0}(z)$, so ergibt sich für $z \rightarrow \mathrm{I},|z|<\mathrm{I}$ :

$$
\pi|f(z)| \leqq\left(k_{+}+k_{-}+2 \varepsilon+\varepsilon_{10}(z)\right) \lg \frac{\mathrm{I}}{|\mathrm{I}-z|},|\arg (\mathrm{I}-z)| \leqq \frac{\pi}{2}-\delta, \delta>0 .
$$

Daher ergibt sich wegen der Willkür von $\varepsilon$ für das aus dem Innern von $E_{z}$ im Winkel gegen I konvergierende $z$

$$
\overline{\operatorname{Lim}} \pi \frac{|f(z)|}{-\lg |1-z|} \leqq k_{+}+k_{-} .
$$

Ganz analog ergibt sich unter Benutzung der Relation $(35,7)$, wenn $z$ aus $E_{z}$ im Winkel gegen I konvergiert,

$$
(39,7) \quad \overline{\operatorname{Lim}}\left(\frac{\pi}{(n+\mathrm{I}) !}(\mathrm{I}-|z|)^{n}\left|f^{(n)}(z)\right|-k_{+} K_{n}(\psi)-k_{-} K_{n}(-\psi)\right) \leqq 0
$$

mit

$$
K_{n}(\psi)=\frac{\cos ^{n} \psi}{n}+\operatorname{tg} \psi \int_{-\psi}^{\frac{\pi}{2}} \cos ^{n} x d x
$$

40. Berücksichtigung der Gleichmässigkeit und allgemeiner Fall der Durchschnittsendlichkeit. Wir haben bei der obigen Herleitung offenbar nur benutzt, dass

$$
\overline{\operatorname{Lim}} \frac{|\Phi(\vartheta)|}{\vartheta} \leqq k_{+} \text {für } \vartheta \downarrow o, \overline{\operatorname{Lim}} \frac{|\Phi(\vartheta)|}{|\vartheta|} \leqq k_{-} \text {für } \vartheta \nmid o
$$

gilt, und es ist ferner aus der Herleitung klar, dass die Relationen $(39,6)$ und $(39,7)$ gleichmässig für alle Funktionen $\chi(\vartheta)$ gelten, für die die Relationen (40, I) gleichmässig gelten - da dann zu einem gegebenen $\varepsilon$ das zugehörige $\delta$ gleichmässig gefunden werden kann —, und die darüber hinaus gleichmässig beschränkt sind, da dann auch die Schranke $C$ für $|\Psi(\vartheta)|$ gleichmässig wählbar ist. 
Über den Habitus der konformen Abbildung am Rande des Abbildungsbereiches. 143

Wir wollen nun die oben zugrunde gelegten Voraussetzungen folgendermassen erweitern: $\chi(\vartheta)$ soll wieder im Intervall $(-\pi, \pi)$ nach LEBesgov integrabel sein und es soll ferner, $\Phi(\vartheta)=\int_{0}^{\vartheta} \chi(\vartheta) d \vartheta$ gesetzt, gelten:

$(40,2)$

$$
\begin{aligned}
& \operatorname{\operatorname {Lim}}\left|\frac{\Phi(\vartheta)}{\vartheta}-g_{+}\right| \leqq k_{+} \text {für } \vartheta \downarrow 0 ; \\
& \overline{\operatorname{Lim}}\left|\frac{\Phi(\vartheta)}{\vartheta}-g_{-}\right| \leqq k_{-} \text {für } \vartheta \uparrow o .
\end{aligned}
$$

$(40,3)$

Mit andern Worten, für $\vartheta=0$ sollen die rechtsseitigen derivierten Zahlen von $\boldsymbol{\Phi}(9)$ im Intervall $\left\langle g_{+}-k_{+}, g_{+}+k_{+}\right\rangle$und die linksseitigen im Intervall $\left\langle g_{-}-k_{-}, g_{-}+k_{-}\right\rangle$liegen. $g_{+}, g_{-}, k_{+}, k_{-}$sollen dabei endliche Zahlen sein, die beiden letzteren nicht negativ. Es sei ferner $g_{+}-g_{-}=\Delta$ gesetzt.

Da die Addition einer Konstanten zu $\chi(\vartheta)$ sich nur darin äussert, dass $f(z)$ sich um eine additive Konstante ändert, dürfen wir, ohne $\Delta, k_{+}$und $k_{-}$zu ändern, voraussetzen, dass $g_{+}+g_{-}$gleich Null ist, so dass $g_{+}=-g_{-}=\frac{\Delta}{2}$ gilt. Nun besitzt aber nach Satz II der Realteil von $-\frac{\Delta}{\pi} i g_{0}(z)$ auf der oberen bzw. unteren Hälfte von $E_{z}$ die Randwerte $\frac{A}{2}$ bzw. $-\frac{A}{2}$. Daher gelten für $(40,4)$

$$
f^{*}(z)=f(z)+\frac{\Delta}{\pi} i g_{0}(z)
$$

die Voraussetzungen (4O, I), unter denen die Relationen $(39,6)$ und $(39,7)$ gelten. Somit erhalten wir wegen $($ IO, 4$),($ IO, 5)

$$
\left.\overline{\operatorname{Lim}} \mid \frac{\pi f(z)}{-\lg (1-z)}+i\right\lrcorner \mid \leqq k_{+}+k_{-},
$$

$(40,6) \widetilde{\operatorname{Lim}}\left\{\left|\frac{\pi}{(n+\mathrm{I}) !}(\mathrm{I}-|z|)^{n} f^{(n)}(z)+\frac{i \Delta e^{i n \psi}}{n(n+\mathrm{I})} \cos ^{n} \psi\right|-k_{+} K_{n}(\psi)-k_{-} K_{n}(-\psi)\right\} \leqq \mathrm{O}$

und die Relationen $(40,5),(40,6)$ gelten offenbar gleichmässig für alle Funktionen $\chi(\vartheta)$, für die die Relationen $(40,2)$ und $(40,3)$ gleichmässig gelten und die darüber hinaus gleichmässig beschränkt sind. 
4I. Einfluss gewisser Variabelntransformationen auf die Durchschnittsendlichkeit. Satz XV. Es sei $f(x)$ in einer Umgebung von $x=0$ fïr $x>0$ nach LEBESGUE integrierbar; und es möge für $x \downarrow \mathrm{O}$

$$
\overline{\operatorname{Lim}} \frac{\mathrm{I}}{x} \int_{0}^{x} f(x) d x=G, \underline{\operatorname{Lim}} \frac{\mathbf{I}}{x} \int_{0}^{x} f(x) d x=g
$$

sein.

Es sei $\varphi(y)$ eine für $y \geqq 0$ zweimal stetig differenzierbare Funktion mit $\varphi(0)=0, \varphi^{\prime}(0)>0, \varphi(y)>0$, und es möge $\varphi(y)$ die Eigenschaft haben, dass vermöge der Abbildung $x=\varphi(y)$ in der Umgebung des Nullpunktes einer $y$-Nullmenge eine $x$-Nullmenge entspricht und umgekehrt. (Diese letzte Voraussetzung ist sicher erfüllt, wenn $\varphi$ eine in der Umgebung von $y=0$ reguläre analytische Funktion von $y$ mit $\varphi^{\prime}(\mathrm{o}) \neq \mathrm{o}$ ist $)$ Dann besitzt für $y$ \o der Quotient

$$
\frac{\mathbf{I}}{y} \int_{0}^{y} f(\varphi(y)) d y
$$

wiederum die Unbestimmtheitsgrenzen $G, g$.

Beweis: Aus den über $\varphi(y)$ gemachten Voraussetzungen folgt

$$
\frac{\mathrm{I}}{x} \int_{0}^{x} f(x) d x=\frac{\mathrm{I}}{\varphi(y)} \int_{0}^{y} f(\varphi(y)) \varphi^{\prime}(y) d y \sim \frac{\mathrm{I}}{\varphi^{\prime}(\mathrm{o})} \frac{\mathrm{I}}{y} \int_{0}^{y} f(\varphi(y)) \varphi^{\prime}(y) d y .
$$

Es genügt daher zu beweisen, dass die Differenz

$$
\frac{\mathrm{I}}{y \varphi^{\prime}(\mathrm{o})} \int_{0}^{y} f(\varphi(y)) \varphi^{\prime}(y) d y-\frac{\mathrm{I}}{y} \int_{0}^{y} f(\varphi(y)) d y
$$

für $y\rfloor$ gegen Null konvergiert. Der absolute Betrag dieser Differenz ist aber höchstens gleich

$$
\frac{\mathrm{I}}{y\left|\varphi^{\prime}(\mathrm{o})\right|} \int_{0}^{y}|f(\varphi(y))|\left|\varphi^{\prime}(y)-\varphi^{\prime}(\mathrm{o})\right| d y
$$

und dies ist, wegen

$$
\varphi^{\prime}(y)-\varphi^{\prime}(0)=O(y)
$$


Über den Habitus der konformen Abbildung am Rande des Abbildungsbereiches. 145 wiederum gleich

$$
O\left(\int_{0}^{y}|f(\varphi(y))| d y\right)=o(\mathrm{I})
$$

da $f(\varphi(y))$ nach LEBEsgue integrierbar ist.

Aus unserem Beweis ergibt sich zugleich ohne weiteres der folgende

Zusatz zum Satz $X V$. Es sei $f(x)$ nach LEBesgon integrabel in einem Intervall $x_{1} \leqq x \leqq x_{2}$ und es mögen für jeden Punkt $x_{0}$ einer in $\left\langle x_{1}, x_{2}\right\rangle$ enthaltenen Punktmenge $M$ die Relationen gelten:

$(4 \mathrm{I}, \mathrm{I})$

$$
\overline{\operatorname{Lim}} \frac{\mathrm{I}}{x-x_{0}} \int_{x_{0}}^{x} f(x) d x=G\left(x_{0}\right)
$$

$$
\operatorname{Lim} \frac{\mathrm{I}}{x-x_{0}} \int_{x_{0}}^{x} f(x) d x=g\left(x_{0}\right)
$$

für $x \rightarrow x_{0}$, wobei für jeden Punkt $x_{0}$ entweder $x \downarrow x_{0}$ oder $x \uparrow x_{0}$ oder beides - und dann evtl. mit verschiedenen $G, g-$ gelten soll. Gelten nun diese Relationen gleichmässig für alle Punkte $x_{0}$ von $M$, werden über die Funktion $x=\varphi(y)$ die analogen Voraussetzungen wie im Satz $X V$ gemacht und wivd $\varphi^{\prime}(y)$ dariiber hinaus als positiv vorausgesetzt, so gelten für die Funktion $f(\varphi(y))$ die zu $(4 \mathrm{I}, \mathrm{I}),(4 \mathrm{I}, 2)$ analogen Relationen gleichmässig auf der entsprechenden $y$-Menge.

42. Transformation von $z=\mathrm{I}$ auf $z=e^{i 9_{0}}$. Wir haben in den Nrn. 39, 40 $\vartheta$ von links bzw. von rechts gegen o gehen lassen. Es ist aber klar, dass genau analoge Resultate gelten, wenn sich die Voraussetzungen $(40,2),(40,3)$ auf $\vartheta \downarrow \vartheta_{0}$ bzw. $\vartheta \uparrow \vartheta_{0}$ beziehen, wobei dann $\vartheta$ in den Nennern durch $\vartheta$ durch $\Phi(\vartheta)-\Phi\left(\vartheta_{0}\right)$ zu ersetzen ist, so dass also nunmehr für $\vartheta==\vartheta_{0}$ die rechtsseitigen derivierten Zahlen von $\Phi(\vartheta)$ im Intervall $\left\langle g_{+}-k_{+}, g_{+}+k_{+}\right\rangle$und die linksseitigen im Intervall $\left\langle g_{-}-k_{-}, g_{-}+k_{-}\right\rangle$liegen. Dann multipliziert sich in $(40,6) f^{(n)}(z)$ mit $e^{i n \vartheta_{0}}$, und zum Faktor $(\mathrm{I}-|z|)^{n}$ kommt noch der Faktor $e^{i n \vartheta_{0}}$ hinzu. $(40,5)$ und $(40,6)$ gelten aber nummehr, wenn $z$ im Winkel aus dem Innern von $E_{z}$ gegen $e^{i 9_{0}}$ strebt. Dabei ist natürlich unter $\psi$ jetzt $-\arg \left(\mathrm{I}-z e^{-i 9_{0}}\right)$ $\mathrm{zu}$ verstehen, und in $(40,5)$ ist $\lg (\mathrm{I}-z)$ durch $\lg \left(\mathrm{I}-z e^{-i 9_{0}}\right)$ zu ersetzen.

19-34472. Acta mathematica. 64. Imprimé le 1 novembre 1934. 
43. Dritte Erweiterung des Satzes I. Nunmehr ist ohne weiteres eine Erweiterung des Satzes XIII auf den Fall der Durchschnittsendlichkeit möglich, wobei wir zugleich die geometrischen Voraussetzungen des Satzes XII zugrunde legen. Wir formulieren unser Resultat wie folgt:

Satz XVI. Es sei $f(z)$ eine fiir $|z|<$ I reguläre analytische Funktion, und es sei $M$ eine solche Punktmenge auf $E_{z}$, dass für ein festes $\varepsilon>0$ in der $\varepsilon$-Umgebung jedes Punktes von $M$ innerhalb $K_{z}|\Re f(z)|$ gleichmässig beschränkt $\leqq C$ ist. Die dann nach FAто in der $\varepsilon$-Umgebung der Menge $M$ auf $E_{z}$ bis auf eine Nullmenge beim Grenzübergang. im Winkel existierende und gleichmässig beschränkte Randfunktion von $\mathfrak{R} f(z)$ sei mit $\chi(\vartheta)$ bezeichnet. Für jeden Punkt $e^{i \vartheta_{0}}$ von $M$ mögen nun die vier derivierten Zahlen von $\int_{\vartheta_{0}}^{\vartheta} \chi(\boldsymbol{g}) d \boldsymbol{g}$ im Intervall $\left\langle g_{+}\left(\boldsymbol{\vartheta}_{0}\right)-k_{+}\left(\boldsymbol{\vartheta}_{0}\right)\right.$, $\left.g_{+}\left(\vartheta_{0}\right)+k_{+}\left(\vartheta_{0}\right)\right\rangle$ fïr die rechtsseitigen, im Intervall $\left\langle g_{-}\left(\boldsymbol{\vartheta}_{0}\right)-k_{-}\left(\vartheta_{0}\right), g_{-}\left(\boldsymbol{\vartheta}_{0}\right)+\right.$ $\left.+k_{-}\left(\vartheta_{0}\right)\right\rangle$ für die linksseitigen derivierten Zahlen liegen, und es mögen überdies die Relationen

$$
\overline{\operatorname{Lim}}\left|\frac{\mathrm{I}}{\boldsymbol{\vartheta}-\boldsymbol{\vartheta}_{0}} \int_{\vartheta_{0}}^{\vartheta} \chi(\vartheta) d \vartheta-g_{+}\left(\vartheta_{0}\right)\right| \leqq k_{+}\left(\vartheta_{0}\right) \text { für } \vartheta \downarrow \vartheta_{0}
$$

$$
\overline{\operatorname{Lim}}\left|\frac{\mathrm{I}}{\vartheta-\vartheta_{0}} \int_{\vartheta_{0}}^{\vartheta} \chi(\vartheta) d \vartheta-g_{-}\left(\vartheta_{0}\right)\right| \leqq k_{-}\left(\vartheta_{0}\right) \text { für } \vartheta \uparrow \vartheta_{0}
$$

gleichmässig für alle $\vartheta_{0}$ auf $M$ gelten. Dann gilt, wenn $z$ aus dem Innern von $E_{z}$ im Winkel gegen die Punkte von $M$ strebt, gleichmässig fïr alle Punkte $e^{i 9_{0}}$ von $\boldsymbol{M}$ und fïr $\left|\arg \left(\mathrm{I}-z e^{-i \vartheta_{0}}\right)\right| \leqq \frac{\pi}{2}-\delta, \delta>\mathrm{o}, \Delta\left(\vartheta_{0}\right)=g_{+}\left(\vartheta_{0}\right)-g_{-}\left(\vartheta_{0}\right)$ gesetzt:

$$
\overline{\operatorname{Lim}}\left|\frac{\pi f(z)}{-\lg \left(\mathrm{I}-z e^{-i \bar{\vartheta}_{0}}\right)}+i \Delta\left(\vartheta_{0}\right)\right| \leqq k_{+}\left(\vartheta_{0}\right)+k_{-}\left(\vartheta_{0}\right)
$$

$(43,4) \quad \overline{\operatorname{Lim}}\left\{\left|\frac{\pi}{(n+\mathrm{I}) !}(\mathrm{I}-|z|)^{n} e^{i n \vartheta_{0}} f^{(n)}(z)+\frac{i \Delta\left(9_{0}\right) e^{i n \psi}}{n(n+\mathrm{I})} \cos ^{i} \psi\right|-\right.$

$$
\left.-k_{+}\left(\vartheta_{0}\right) K_{n}(\psi)-k_{-}\left(\vartheta_{0}\right) K_{n}(-\psi)\right\} \leqq \text { о }
$$

wo $\psi=-\arg \left(\mathrm{I}-z e^{-i 9_{0}}\right)$ ist und $\boldsymbol{K}_{n}(\psi)$ die Bedeutung $(39,8)$ hat. 
Über den Habitus der konformer Abbildung am Rande des Abbildungsbereiches. 147

44. Beweis: Es sei $C_{1}>C$ eine positive Konstante derart, dass $|\Re f(z)|<C_{1}$ für $|z| \leqq I-\frac{\varepsilon}{4}$ ist. Man bilde wie beim Beweis von Satz XII endlich viele zyklisch angeordnete abgeschlossene Kreisbogen $B_{1}, \ldots, B_{m}$ auf $E_{z}$, die die Menge $M$ samt ihren Häufungsstellen ganz im Innern enthalten und selbst im Innern der $\frac{\varepsilon}{4}$-Umgebung von $M$ enthalten sind. Ist allgemein $B_{\mu}^{*}$ der zwischen $B_{\mu}$ und $B_{\mu+1}$ bzw. zwischen $B_{m}$ und $B_{1}$ liegende Kreisbogen von $E_{z}$, so lassen sich die Endpunkte jedes $B_{\mu}^{*}$ durch einen Kreisbogen $\alpha_{\mu}$ verbinden, der bis auf seine Endpunkte ganz innerhalb von $E_{z}$ verläuft und auf dem $|\Re f(z)|$ gleichmässig beschränkt $\leqq C_{1}$ ist. Es möge nun die Funktion $w=F(z)$ das von den Kreisbögen $B_{\mu}$ und $\alpha_{\mu}$ begrenzte Flächenstück konform auf das Innere von $E_{w}$ abbilden, etwa so, dass ein bestimmtes Linienelement im Nullpunkt wiederum in ein solches übergeht. Bekanntlich ist $F(z)$ regulär innerhalb der Kreisbogen $B_{\mu}$ und es gibt zwei positive Konstanten $c_{1}, c_{2}, c_{1}<c_{2}$, derart, dass der absolute Betrag von $F^{\prime}(z)$ in einer $\varepsilon_{1}$-Umgebung von $M$ zwischen $c_{1}$ und $c_{2}$ liegt. Durch unsere Transformation möge nun $f(z)$ in $f_{1}(w)$ übergehen, so dass $f(z)=f_{1}(\boldsymbol{F}(z))$ ist. Die Randfunktion von $\Re f_{1}(w)$ auf $E_{w}$ sei mit $\chi_{1}(\vartheta), w=r e^{i \vartheta}$, bezeichnet. Dann ist $\chi_{1}(\vartheta)=\chi(\varphi(\vartheta))$, wo $\varphi(\vartheta)$ innerhalb der Bildbogen der Bogen $B_{1}, \ldots, B_{m}$ monoton wachsend ist und eine positive Ableitung besitzt, deren Werte auf der ganzen Bildmenge $M_{1}$ von $M$ zwischen zwei positiven Schranken $c_{3}$ und $c_{4}, c_{3}<c_{4}$, enthalten sind. Entspricht einem Punkte $\vartheta_{0}$ von $M$ ein Punkt $\vartheta_{0}^{\prime}$ von $M_{1}$, so folgt aus dem Satze XV, dass die vier derivierten Zahlen von $\int_{\vartheta_{0}^{\prime}}^{\vartheta} \chi_{1}(\vartheta) d \vartheta$ für $\vartheta=\vartheta_{0}^{\prime}$ in den Intervallen $\left\langle g_{+}\left(\vartheta_{0}\right)-k_{+}\left(\vartheta_{0}\right), g_{+}\left(\vartheta_{0}\right)+k_{+}\left(\vartheta_{0}\right)\right\rangle,\left\langle g_{-}\left(\vartheta_{0}\right)-k_{-}\left(\vartheta_{0}\right), g_{-}\left(\vartheta_{0}\right)+\right.$ $\left.+k-\left(\vartheta_{0}\right)\right\rangle$ liegen.

Andererseits ist $\left|\chi_{1}(\vartheta)\right| \leqq C_{1}$ auf der ganzen Kreislinie $E_{w}$. Daher können unsere oben hergeleiteten Resultate $(40,5),(40,6)$ (vgl. Nr. 42) auf jeden Punkt $\vartheta_{0}^{\prime}$ der Menge $M_{1}$ und die Funktion $f_{1}(w)$ angewandt werden und gelten gleichmässig für alle Punkte dieser Menge. Dabei steht allerdings in den $(40,5)$ und $(40,6)$ entsprechenden Relationen zunächst $-\lg \left(\mathrm{I}-w e^{-i \vartheta_{0}}\right)$ bzw. $(\mathrm{I}-|w|)^{n} e^{i n \vartheta_{0}^{\prime}}$, wo $w$ der Bildpunkt von $z$ innerhalb $E_{w}$ ist. Nun folgt aber aus der Existenz einer absolut nach oben und unten gleichmässig positiv beschränkten Ableitung von $F(z)$ in allen Punkten $e^{i \vartheta_{0}}$ von $M$ und einer gewissen Umgebung von $M$ auf $E_{z}$ erstens, dass 


$$
\lg \left(\mathrm{I}-w e^{i 9^{\prime}}\right) \sim \lg \left(\mathrm{I}-z e^{i \vartheta_{0}}\right),
$$

und zwar gleichmässig in einer Umgebung von $M$ gilt. Daher ergibt sich $(43,3)$ ohne weiteres. Aus dem gleichen Grunde ist, wenn $z$ gegen $e^{i \vartheta_{0}}$ aus $K_{z}$ im Winkel strebt,

$$
\frac{\mathrm{I}-|w|}{\mathrm{I}-|z|} \rightarrow\left|F^{\prime}\left(e^{i \vartheta_{0}}\right)\right|
$$

Ferner folgt aus $f(z)=f_{1}(F(z))$ durch $n$-malige Differentiation

$$
f^{(n)}(z)=f_{1}^{(n)}(w)\left(F^{\prime}(z)\right)^{n}+O\left(f_{1}^{\prime}(w)\right)+O\left(f_{1}^{\prime \prime}(w)\right)+\cdots+O\left(f_{1}^{(n-1)}(w)\right),
$$

oder, wenn man mit $(\mathrm{I}-|z|)^{n}$ multipliziert und $(44,2)$ beachtet,

$$
(\mathrm{I}-|z|)^{n} f^{(n)}(z)=(\mathrm{I}-|w|)^{n} f_{1}^{(n)}(w) e^{i n \text { arg } F^{\prime}\left(e^{i \theta_{0 j}}\right.}+O(\mathrm{I}-|z|) .
$$

Andererseits wird durch die konforme Abbildung $w=F(z)$ die zur Kreislinie $E_{z}$ im Punkte $e^{i 9_{0}}$ tangierende Richtung in die Richtung der entsprechend orientierten Tangente zur Kreislinie $E_{w}$ im Punkte $e^{i \vartheta^{\prime}}$ übergeführt, so dass

$$
\arg F^{\prime}\left(e^{i \vartheta_{0}}\right) \equiv \vartheta_{0}^{\prime}-\vartheta(\bmod 2 \pi)
$$

sein muss. Daher ergibt sich schliesslich

$$
(\mathrm{I}-|w|)^{n} f_{\mathrm{i}}^{(n)}(w) e^{i n \vartheta_{0}^{\prime}}=(\mathrm{I}-|z|)^{n} f^{(n)}(z) e^{i n 9_{0}}+O(\mathrm{I}-|z|) .
$$

Setzen wir dies ein, so ergibt sich aus dem auf die $w$-Ebene bezüglichen Resultat die Formel $(43,4)$.

45. Zusätze zum Satz XVI. Wir nehmen nun an, dass die Funktion $\chi(9)$ auf einem abgeschlossenen Bogen $\Gamma$ von $E_{z}$ durchweg beschränkt ist, und zwar mögen ihre Werte innerhalb eines Intervalls von der Länge $D$ liegen. Dann kann man gleichmässig für alle Punkte $\vartheta_{0}$ von $\Gamma k_{+}=k_{-}=\frac{D}{2}$ setzen und als $g_{+}=g_{-}$den Mittelpunkt jenes Intervalls annehmen, so dass $\Delta=0$ ist. Betrachten wir dann nur radiale Annäherung an die inneren Punkte von $\Gamma$, so kann in $(43,3),(43,4) \psi=0$ gesetzt werden, und man erhält, da $K_{n}(0)=\frac{\mathrm{I}}{n}$ ist,

$$
\begin{aligned}
& \overline{\operatorname{Lim}} \frac{|f(z)|}{-\lg (\mathrm{I}-|z|)} \leqq \frac{D}{\pi}, \\
& \overline{\operatorname{Lim}}(\mathrm{I}-|z|)^{n}\left|f^{(n)}(z)\right| \leqq \frac{(n-\mathrm{I}) !(n+\mathrm{I}) D}{\pi} .
\end{aligned}
$$


Über den Habitus der konformen Abbildung am Rande des Abbildungsbereiches. 149

Da aber diese Relationen für jeden ganz innerhalb $\Gamma$ liegenden abgeschlossenen Bogen $\Gamma^{*}$ gleichmässig gelten, gelten sie auch gleichmässig, wenn $z$ aus dem Innern von $E_{z}$ allseitig gegen $I^{*}$ strebt. So erhalten wir den

Zusatz I zum Satze XVI. Ist unter den Voraussetzungen des Satzes XVI die Schwankung von $\chi(9)$ auf einem in $M$ liegenden Bogen $\Gamma$ von $E_{z}$ höchstens gleich $D$, so gilt, wenn $z$ gegen einen ganz innerhalb von $\Gamma$ liegenden abgeschlossenen Teilbogen $\Gamma^{*}$ aus dem Innern von $E_{z}$ allseitig strebt:

$(45, I)$

$$
\overline{\operatorname{Lim}} \frac{|f(z)|}{-\lg (\mathbf{I}-|z|)} \leqq \frac{D}{\pi}
$$

$$
\overline{\operatorname{Lim}}(\mathrm{I}-|z|)^{n}\left|f^{(n)}(z)\right| \leqq \frac{(n-\mathrm{I}) !(n+\mathrm{I}) D}{\pi} \text {. }
$$

Insbesondere gelten die Relationen $(45, \mathrm{I})$ und $(45,2)$, wenn $z$ allseitig gegen einen Punkt $e^{i \vartheta_{0}}$ strebt, falls $\mathfrak{R} f(z)$ in einer Umgebung von $e^{i 9_{0}}$ beschränkt ist und unter. $D$ die Schwankung von $\chi(\vartheta)$ im Punkte $\vartheta_{0}$ verstanden wird.

Wir betrachten andererseits unter den Voraussetzungen von Zusatz I ein Punktepaar $z_{1}, z_{2}$, das aus dem Innern von $E_{z}$ gegen den Bogen $\Gamma^{*}$ derart konvergiert, dass dabei

$$
\frac{z_{2}-z_{1}}{I-\left|z_{1}\right|} \rightarrow \mathrm{O}
$$

gilt. Wegen $1-\left|z_{1}\right|-\left|z_{2}-z_{1}\right| \leqq \mathrm{I}-\left|z_{2}\right| \leqq \mathrm{I}-\left|z_{1}\right|+\left|z_{2}-z_{1}\right|$ folgt aus $(45,3)$

$$
\frac{\mathrm{I}-\left|z_{2}\right|}{\mathrm{I}-\left|z_{1}\right|} \rightarrow \mathrm{I}, \quad \frac{z_{2}-z_{1}}{\mathrm{I}-\left|z_{2}\right|} \rightarrow \mathrm{O}
$$

Nun gilt, wegen $(45,2)$ mit $n=\mathrm{I}$,

$$
\left|f\left(z_{2}\right)-f\left(z_{1}\right)\right|=\left|\int_{z_{1}}^{\tilde{z}^{\prime}} f^{\prime}(z) d z\right|=O\left(\frac{\left|z_{2}-z_{1}\right|}{\operatorname{Min}(\mathrm{I}-|z|)}\right)
$$

wo Min $(\mathrm{I}-|z|)$ für die Strecke $z_{1}^{\prime} z_{2}$ zu nehmen ist. Offenbar wird aber die kürzeste Distanz eines Punktes der Strecke $z_{1} z_{2}$ von $E_{z}$ in einem der Endpunkte der Strecke erreicht. Aus $(45,3)$ und $(45,4)$ folgt daher $f\left(z_{2}\right)-f\left(z_{1}\right) \rightarrow 0$. Wir erhalten den 
Zusatz 2 zum Satz XVI. Unter den Voraussetzungen von Zusatz I gilt für ein Punktepaar $z_{1}, z_{2}$, das aus dem Innern von $E_{z}$ so gegen $\Gamma^{*}$ konvergiert, dass $(45,3)$ gilt, die Relation

$$
f\left(z_{2}\right)-f\left(z_{1}\right) \rightarrow \mathrm{o}
$$

und zwar gleichmässig, wenn $(45,3)$ gleichmässig gilt.

Es mögen nun für alle Punkte $e^{i \vartheta_{0}}$ der Menge $M$ die drei Funktionen $k_{+}\left(\vartheta_{0}\right), k_{-}\left(\vartheta_{0}\right), \Delta\left(\vartheta_{0}\right)$ durchweg verschwinden. Dann liefert $(43,4)$ für $n=\mathrm{I}$ die Relation

$$
f^{\prime}(z)=o\left(\frac{\mathrm{I}}{1-|z|}\right)
$$

gleichmässig für alle Punkte $e^{i \vartheta_{0}}$ von $M$. Da aber der Beweis des Satzes XIV nur von dieser Relation Gebrauch macht, bleibt $(38,1)$ auch unter unseren Voraussetzungen richtig, und wir erhalten den

Zusatz 3 zum Satze XVI. Verschwinden unter den Voraussetzungen des Satzes XVI die Grössen $k_{+}\left(\vartheta_{0}\right), k_{-}\left(\vartheta_{0}\right), \Delta\left(\vartheta_{0}\right)$ für alle Punkte von $M$, so gilt, wenn $z_{1}$ und $z_{2}$ aus $K_{z}$ im Winkel gegen einen Punkt $z_{0}=e^{i 9_{0}}$ von $\boldsymbol{M}$ so konvergiert, dass $\left|\frac{z_{0}-z_{2}}{z_{0}-z_{1}}\right|$ zwischen beliebigen aber festen positiven Schranken bleibt,

$$
f\left(z_{2}\right)-f\left(z_{1}\right) \rightarrow o
$$

und diese Relation gilt gleichmässig für die ganze Menge $M$.

Lässt man in der Formulierung von Zusatz 3 die Annahme, dass $\boldsymbol{\Delta}\left(\boldsymbol{\vartheta}_{0}\right)$ verschwindet, fallen, so kann man die folgende Überlegung anstellen. Der Einfachheit halber sei $\vartheta_{0}=0$. Dann genügt die Differenz

$$
f(z)-\frac{A}{i \pi} g_{0}(z)
$$

wo $g_{0}(z)$ die Hilfsfunktion des Satzes II ist, allen Voraussetzungen des Zusatzes 3 zum Satz XVI. Es folgt, wenn $\left|\frac{z_{2}-I}{z_{1}-I}\right|$ zwischen festen positiven Schranken bleibt, wegen $\mathrm{I}+z_{1} \rightarrow 2, \mathrm{I}+z_{2} \rightarrow 2$,

$$
f\left(z_{2}\right)-f\left(z_{1}\right)-\frac{A}{i \pi} \lg \left(\frac{\mathrm{I}-z_{1}}{\mathrm{I}-z_{2}}\right) \rightarrow 0
$$


Über den Habitus der konformen Abbildung am Rande des Abbildungsbereiches. 151 Nun wird $K_{z}$ durch die Transformation $z=\frac{\zeta-\mathrm{I}}{\zeta+\mathrm{I}}$ abgebildet auf die Halbebene $\Re \zeta>0$. Setzen wir

$$
f\left(\frac{\zeta-\mathrm{I}}{\zeta+\mathrm{I}}\right)=F(\zeta)
$$

so liefert $(45,63)$

$$
F\left(\zeta_{2}\right)-F\left(\zeta_{1}\right)+i \frac{\Delta}{\pi} \lg \frac{\zeta_{2}}{\zeta_{1}} \rightarrow 0
$$

wenn $\zeta_{1}$ und $\zeta_{2}$ so ins Unendliche gehen, dass

$$
\left|\arg \zeta_{1}\right| \leqq \frac{\pi}{2}-\delta, \quad\left|\arg \zeta_{2}\right| \leqq \frac{\pi}{2}-\delta, \quad \delta>0
$$

und $o<c_{1} \leqq\left|\frac{\zeta_{2}}{\zeta_{1}}\right| \leqq c_{2}<\infty$ ist.

Wie lauten nun die $(43, I)$ und $(43,2)$ entsprechenden Bedingungen - mit $k_{+}=k_{-}=0$ für $F^{\prime}(\zeta)$ ? Wir setzen $\vartheta_{0}=0$ voraus. Dann entspricht dem Punkt $z=e^{i \vartheta}$ von $E_{z}$ der Punkt it auf der imaginären Axe mit $t=\operatorname{ctg} \frac{\vartheta}{2}$. Es sei nun $u=\frac{\mathrm{I}}{t}, \mathfrak{R} F(i t)=\psi(u)$. Wegen $u=\operatorname{tg} \frac{\vartheta}{2}$ ist der Satz XV anwendbar, so dass die Relationen $(43, \mathrm{I})$ und $(43,2)$ in

$$
\frac{I}{u} \int_{0}^{u} \psi(u) d u \rightarrow\left\{\begin{array}{l}
g_{+} \text {für } u \downarrow o \\
g_{-} \text {für } u \uparrow o
\end{array}\right.
$$

übergehen. Diese beiden Relationen gehen aber, wenn nunmehr $t=\frac{\mathrm{I}}{u}$ als neue Integrationsvariable eingeführt wird, in die Bedingungen

$$
\begin{aligned}
& t \int_{i}^{\infty} \Re F(i t) \frac{d t}{t^{2}} \rightarrow g_{+}, \quad t \uparrow \infty, \\
& t \int_{t}^{-\infty} \Re F(i t) \frac{d t}{t^{2}} \rightarrow g_{-}, \quad t \downarrow-\infty
\end{aligned}
$$


uber. $^{30}$ Setzen wir endlich $F(\zeta)=\frac{\mathrm{I}}{i} \lg G(\zeta)$, so ergibt sich die folgende Formulierung:

Korollar zum Zusatz 3. Es sei $G(\zeta)$ eine für $\Re \zeta>0$ reguläre analytische nirgends verschwindende Funktion, für die im Bereich $\Re \zeta>0,|\zeta|>C_{0}$ die Gesamtschwankung von $\arg G(\zeta)$ gleichmässig beschränkt ist. Die dann in den Punkten $\zeta=i t$ der imaginären Axe nach Fatou für hinreichend grosse $|t|$ bis auf eine $t$-Nullmenge beim Grenzübergang im Winkel existierende und gleichmässig beschränkte Grenzfunktion von arg $G(\zeta)$ sei mit $\arg G(i t)$ bezeichnet. Gilt dann für $t \uparrow \infty$ bzw. $t \downarrow-\infty$

$$
t \int_{t}^{\infty} \arg G(i t) \frac{d t}{t^{2}} \rightarrow g_{+}, \quad t \int_{t}^{-\infty} \arg G(i t) \frac{d t}{t^{2}} \rightarrow g_{-},
$$

so gilt, $g_{+}-g_{-}=\Delta$ gesetzt, wenn $\zeta_{1}$ und $\zeta_{2}$ so ins Unendliche konvergieren, dass

$$
\begin{gathered}
\left|\arg \zeta_{1}\right| \leqq \frac{\pi}{2}-\delta, \quad\left|\arg \zeta_{2}\right| \leqq \frac{\pi}{2}-\delta \\
c_{1} \leqq\left|\frac{\zeta_{1}}{\zeta_{2}}\right| \leqq c_{2}
\end{gathered}
$$

für beliebig aber fest gewählte positive $\delta, c_{1}, c_{2}$ ist, die Relation

$$
\frac{G\left(\zeta_{2}\right)}{G\left(\zeta_{1}\right)} \sim\left(\frac{\zeta_{2}}{\zeta_{1}}\right)^{31}
$$

Zusatz 4 zum Satze XVI. Unter den Voraussetzungen des Satzes XVI gilt, wenn $z$ im Winkel gegen die Punkte $e^{i \vartheta_{0}}$ von $M$ strebt,

30 An sich involviert die Existenz des LEBEsgueschen Integrals zugleich die absolute Integrierbarkeit des Integranden, so dass, um Aequivalente der Bedingungen $(43,1),(43,2)$ für unendliches Integrationsintervall $\mathrm{zu}$ erhalten, eigentlich noch die Voraussetzung der absoluten Konvergenz des betreffenden Integrals hinzugenommen werden müsste. In unserem Falle wird aber im Satz XVI die Beschränktheit von $\Re f(z)$ in der Nähe von $z=$ I vorausgesetzt. Dem entspricht aber die gleichmässige Beschränktheit von $F(\zeta)$ für hinreichend grosse $\zeta$, sodass die absolute Konvergenz unserer Integrale aus der Messbarkeit der Integranden folgt.

${ }^{31}$ Für $\left|\zeta_{1}\right|=\left|\zeta_{2}\right|$ ergibt sich aus $(45,65)$, wenn auf beiden Seiten die absoluten Beträge verglichen werden, die Relation $\left|G\left(\zeta_{2}\right)\right| \sim\left|G\left(\zeta_{1}\right)\right|$, die in VIsser (I), p. 32, als Satz 4 formuliert wird, unter der speziellen Voraussetzung, dass arg $G(i t) \rightarrow \pm \frac{\pi}{2}$ resp. für $t \rightarrow \pm \infty$ gilt, sodass $A$ insbesondere $=\pi$ ist. 
Über den Habitus der konformen Abbildung am Rande des Abbildungsbereiches. 153

$(45,7) \quad \tilde{\Im} f(z)=\frac{\Delta+\Theta\left(k_{+}\left(\vartheta_{0}\right)+k_{-}\left(\vartheta_{0}\right)\right)}{\pi} \lg \left|\mathrm{I}-z e^{i \vartheta_{0}}\right|+o\left(\lg \left|\mathrm{I}-z e^{i \vartheta_{0}}\right|\right)$,

und unter der Voraussetzung

$\left|\not\left(\vartheta_{0}\right)\right|>k_{+}\left(\vartheta_{0}\right)+k_{-}\left(\vartheta_{0}\right)$

die Formel

$$
\mathfrak{S} f(z) \rightarrow-\operatorname{sgn} \Delta \infty
$$

wobei $-\mathrm{I} \leqq \Theta \leqq \mathrm{I}$ bleibt; $(45,7)$ gilt unter gleichen Voraussetzungen gleichmässig wie der Satz XVI, während $(45,9)$ gleichmässig gilt, wenn ausserdem vorausgesetzt wird, dass $\left|\mathcal{L}\left(\boldsymbol{\vartheta}_{0}\right)\right|-k_{+}\left(\boldsymbol{\vartheta}_{0}\right)-k_{-}\left(\boldsymbol{\vartheta}_{0}\right)$ oberhalb einer festen positiven Schranke bleibt.

Denn aus $(43,3)$ folgt, wenn beide Seiten dieser Gleichung mit $-\lg \left(1-z e^{i g_{0}}\right)$ multipliziert werden,

$$
f(z)=\frac{i \Delta\left(\vartheta_{0}\right)+\Theta_{1}\left(k_{+}\left(\vartheta_{0}\right)+k_{-}\left(\vartheta_{0}\right)\right)}{\pi} \lg \left(\mathrm{I}-z e^{i \vartheta_{0}}\right)+o\left(\lg \left(\mathrm{I}-z e^{i \vartheta_{0}}\right)\right)
$$

mit $\left|\Theta_{1}\right| \leqq I$. Hieraus aber ergibt sich $(45,7)$ sofort, wenn die Imaginärteile auf beiden Seiten verglichen werden und der Imaginärteil von $\Theta_{1}$ mit $\Theta$ bezeichnet wird.

$(45,9)$ folgt aber unter der Voraussetzung $(45,8)$ aus $(45,7)$ sofort.

Es sei endlich betont, dass der Satz XVI und die Zusätze 3 und 4 zu diesem Satz insbesondere dann ohne Weiteres angewandt werden dürfen, wenn bekannt ist, dass $\overline{\operatorname{Lim}} \chi(\boldsymbol{\eta})$ für $\boldsymbol{\vartheta} \downarrow \boldsymbol{\vartheta}_{\mathrm{o}}$ bzw. $\boldsymbol{\vartheta} \uparrow \boldsymbol{\vartheta}_{0}$ im Intervall $\left\langle g_{+}\left(\boldsymbol{\vartheta}_{0}\right)-k_{+}\left(\boldsymbol{\vartheta}_{0}\right), g_{+}\left(\boldsymbol{\vartheta}_{0}\right)+k_{+}\left(\boldsymbol{\vartheta}_{0}\right)\right\rangle$ bzw. im Intervall $\left\langle g_{-}\left(\vartheta_{0}\right)-k_{-}\left(\vartheta_{0}\right), g_{-}\left(\vartheta_{0}\right)+k_{-}\left(\vartheta_{0}\right)\right\rangle$ liegt.

\section{$\S$ ı. Eine Bedingung für die Darstellbarkeit durch das Poissonsche Integral.}

46. Formulierung der Sätze. Bei der Untersuchung von Spitzenabbildungen $(\gamma=0)$ werden wir vom folgenden Satz Gebrauch zu machen haben:

Satz XVII. Es sei $f(z)=U(z)+i V(z)$ eine für $|z|<$ I reguläre analytische Funktion, fü deren Realteil $U(z)$ das Integral

$$
\int_{-\pi}^{\pi} \int_{0}^{1}\left|U\left(r e^{i \vartheta}\right)\right| r d r d \vartheta
$$

20-34472. Acta mathematica. 64. Imprimé le I novembre 1934. 
existiert. Der Imaginärteil $V(z)$ möge, wenn $z$ allseitig aus $K_{z}$ gegen die Punkte $e^{i 9}$ des Einheitskreises konvergiert, mit eventueller Ausnahme eines $\vartheta^{-W e r t e s} \vartheta_{0}$, gegen eine Funktion $\chi(\vartheta)$ konvergieren, die in jedem von $e^{i \vartheta_{0}}$ verschiedenen Punkte des Einheitskreises stetig und auf $E_{z}$ gleichmässig beschränkt sei. Dann hat $f(z)$ die Gestalt

$(46,2) \quad f(z)=\frac{i}{2 \pi} \int_{-\pi}^{\pi} \frac{e^{i \vartheta}+z}{e^{i \vartheta}-z} \chi(\vartheta) d \vartheta+h(z), h(z)=i u \frac{e^{i \vartheta_{0}}+z}{e^{i \vartheta_{0}}-z}+v$, wo $u, v$ gewisse reelle Konstanten sind. - Die Umkehrung ist klar.

Unser Beweis des Satzes XVII reicht zugleich zum Beweis des folgenden allgemeineren Satzes aus:

Satz XVIII: Es sei $e^{i \vartheta_{0}}$ ein Punkt, der im Innern eines offenen Kreisbogens $\Gamma$ von $E_{z}$ liegt. $\sigma$ sei eine Kreisbogensichel, deren Rand erstens aus $\Gamma$ und zweitens aus einem die Endpunkte von $\Gamma$ verbindenden, sonst ganz innerhalb $K_{z}$ verlaufenden Kreisbogens besteht. (Fü) $\Gamma=E_{z}$ ist $\sigma=K_{z}$.) $\quad$ Es sei $f(z)=U(z)+i V(z)$ eine für $|z|<\mathrm{I}$ reguläre analytische Funktion, für deren Realteil $U(z)$ das Integral

$$
\iint_{\sigma}\left|U\left(r \cdot e^{i \vartheta}\right)\right| r d r d \boldsymbol{q}
$$

ïber das Innere von $\sigma$ erstreckt, existiert. Der Imaginärteil $V(z)$ möge, wenn $z$ allseitig aus $K_{z}$ gegen die Punkte $e^{i g}$ von $r$ konvergiert, mit eventueller Ausnahme von $\vartheta=\vartheta_{0}$, gegen eine Funktion $\chi(\vartheta)$ konvergieren, die in jedem von $e^{i \vartheta_{0}}$ verschiedenen Punkte von $\Gamma$ stetig und auf $\Gamma$ gleichmässig beschränkt ist. Dann hat $f(z)$ die Gestalt

$(46,4) \quad f(z)=\frac{i}{2 \pi} \int_{-\pi}^{\pi} \frac{e^{i \vartheta}+z}{e^{i \vartheta}-z} \chi(\vartheta) d \vartheta+h(z), h(z)=i u \frac{e^{i 9_{0}}+z}{e^{i 9_{0}}-z}+v+E(z)$,

wo $\chi(\vartheta)$ fïr $\vartheta$-Werte, für die $e^{i \vartheta}$ ausserhalb $\Gamma$ liegt, gleich $\circ$ zu setzen und $\boldsymbol{E}(z)$ eine in $K_{z}$ und auf $\Gamma$ reguläre analytische Funktion mit $E\left(e^{i 9_{0}}\right)=0$ ist, wobei $E(z)$ auf. $I$ reell ist und für $\Re E(z)$ die auf $(46,3)$ bezügliche Bedingung erfiullt ist.

47. Hilfssätze. Dem Beweis dieser Sätze schicken wir drei einfache Hilfssätze voraus.

Lemma 3. Ist $\chi(\boldsymbol{\vartheta})$ eine im Intervall $(-\pi, \pi)$ beschränkte nach LEBesgut integrierbare Funtion mit $|\chi(\vartheta)| \leqq C$ und bildet man daraus mit Hilfe des Poissonschen Integrals die zugehörige analytische Funktion 
Über den Habitus der konformen Abbildung am Rande des Abbildungsbereiches. 155

$$
f(z)=\frac{\mathrm{I}}{2 \pi} \int_{-\pi}^{\pi} \frac{e^{i \vartheta}+z}{e^{i \vartheta}-z} \chi(\vartheta) d \vartheta=U(z)+i V(z)
$$

so sind fuir $-\pi \leqq \vartheta \leqq \pi$ die Integrale

$(47,2)$

$$
\int_{0}^{1}\left|f\left(r e^{i \vartheta}\right)\right| d r, \quad \int_{0}^{1}\left|U\left(r e^{i \vartheta}\right)\right| d r, \quad \int_{0}^{1}\left|V\left(r e^{i \vartheta}\right)\right| d r
$$

höchstens gleich $\gamma C$, und die Integrale

$(47,3) \int_{-\pi}^{\pi} \int_{0}^{1}\left|f\left(r e^{i \vartheta}\right)\right| r d r d \vartheta, \int_{-\pi}^{\pi} \int_{0}^{1}\left|U\left(r e^{i \vartheta}\right)\right| r d r d \vartheta, \quad \int_{-\pi}^{\pi} \int_{0}^{1}\left|V\left(r e^{i \vartheta}\right)\right| r d r d \vartheta$

höchstens $\gamma_{1} C$, wo $\gamma, \gamma_{1}$ positive absolute Konstanten sind.

Beweis von Lemma 3: Es genügt, die Behauptung nur für die ersten Integrale $(47,2)$ bzw. $(47,3)$ zu beweisen. Ohne Beschränkung der Allgemeinheit darf $C=\mathbf{I}$ angenommen werden. Dann ergibt sich aus $(47, \mathrm{I})$

$$
\left|f\left(r e^{i \vartheta}\right)\right| \leqq \frac{1}{\pi} \int_{-\pi}^{\pi} \frac{d \varphi}{\left|e^{i \varphi}-r e^{i \vartheta}\right|} \leqq \frac{2}{\pi} \int_{0}^{\pi} \frac{d \varphi}{\left|e^{i \varphi}-r\right|}
$$

Hieraus folgt weiter

$$
\int_{0}^{1}\left|f\left(r e^{i \vartheta}\right)\right| d r \leqq \frac{2}{\pi} \int_{0}^{\pi} d \varphi \int_{0}^{1} \frac{d r}{\sqrt{(r-\cos \varphi)^{2}+\sin ^{2} \varphi}} .
$$

Hier ergibt sich für das innere Integral, wenn man $r-\cos \varphi=x \sin \varphi$ setzt,

$$
\begin{array}{r}
\int_{0}^{1} \frac{d r}{\sqrt{(r-\cos \varphi)^{2}+\sin ^{2} \varphi}}=\int_{-\operatorname{ctg} \varphi}^{\operatorname{tg} \frac{\varphi}{2}} \frac{d x}{\sqrt{x^{2}+\mathrm{I}}}=\lg \left(\sqrt{x^{2}+\mathrm{I}}+x\right) \int_{-\operatorname{ctg} \varphi}^{\operatorname{tg} \frac{\varphi}{2}}= \\
=\lg \left(\frac{\mathrm{I}}{\cos \frac{\varphi}{2}}+\operatorname{tg} \frac{\varphi}{2}\right)-\lg \left(\frac{\mathrm{I}}{\sin \varphi}-\operatorname{ctg} \varphi\right) .
\end{array}
$$


Setzen wir daher

$(47,4)$

$$
\frac{2}{\pi} \int_{0}^{\pi} \lg \frac{I+\sin \frac{\varphi}{2}}{\sin \frac{\varphi}{2}} d \varphi=\gamma
$$

so folgt

$(47,5)$

$$
\int_{0}^{1}\left|f\left(r e^{i \vartheta}\right)\right| d r \leqq \gamma
$$

und damit ist die auf $(47,2)$ bezügliche Behauptung bewiesen. Aus $(47,5)$ folgt aber

$$
(47,6) \quad \int_{-\pi}^{\pi} \int_{0}^{1}\left|f\left(r e^{i \vartheta}\right)\right| \cdot d r d \vartheta \leqq \int_{-\pi}^{\pi} \gamma d \vartheta=2 \pi \gamma=\gamma^{\prime},
$$

womit auch die auf das erste Integral $(47,3)$ bezügliche Behauptung bewiesen ist.

Lemma 4. Es gilt fïr $|z| \uparrow \mathrm{I}$

$$
\Phi(z)=\int_{-\pi}^{\pi} \frac{d \vartheta}{\left|z-e^{i \vartheta}\right|}=2 \lg \frac{\mathrm{I}}{\mathrm{I}-|z|}+O(\mathrm{I})
$$

Beim Beweis darf offenbar $z$ positiv $=|z|$ vorausgesetzt werden. Dann aber nimmt unser Integral die Gestalt

$$
\int_{-\pi}^{\pi} \frac{d \vartheta}{\sqrt{z^{2}-2 z \cos \vartheta+\mathrm{I}}}
$$

an. Dies ist ein elliptisches Integral, so dass man die Behauptung auch der Theorie der elliptischen Integrale unmittelbar entnehmen kann. Sie lässt sich aber leicht direkt verifizieren. Es sei $z=\mathrm{I}-h, h>0$. Dann verwandelt $\operatorname{sich}(47,7)$ in

$$
2 \int_{0}^{\pi} \frac{d y}{\sqrt{h^{2}+4(\mathrm{I}-h) \sin ^{2} \frac{9}{2}}}
$$


Über den Habitus der konformen Abbildung am Rande des Abbildungsbereiches. 157 Führen wir hier $\sin \frac{\vartheta}{2}=x$ als neue Variable ein, so geht $(47,7)$ über in

$$
4 \int_{0}^{1} \frac{d x}{\sqrt{\mathrm{I}-x^{2}} \sqrt{h^{2}+4(\mathrm{I}-h) x^{2}}}=4 \int_{0}^{\frac{1}{2}}+4 \int_{\frac{1}{2}}^{1} .
$$

Das zweite Integral ist aber offenbar $O(\mathrm{I})$ für $h \downarrow$ o. Im ersten aber ändern wir das Integral nur um $O(\mathrm{I})$, wenn wir $\sqrt{\mathrm{I}-x^{2}}$ durch I ersetzen, da I $-\frac{\mathrm{I}}{\sqrt{\mathrm{I}-x^{2}}}=O\left(x^{2}\right)$ für $x \downarrow \circ$ ist. Führen wir dann $y=\frac{2 x}{h}$ als neue Variable ein, so ergibt sich

$$
\Phi(z)=2 \int_{0}^{\frac{1}{h}} \frac{d y}{\sqrt{\mathrm{I}+(\mathrm{I}-h) y^{2}}}+O(\mathrm{I}) \text {. }
$$

Hier ist aber das unbestimmte Integral gleich

$$
\frac{\mathrm{I}}{\sqrt{\mathrm{I}-h}} \lg \left(\sqrt{\mathrm{I}-h} y+\sqrt{\mathrm{I}+(\mathrm{I}-h) y^{2}}\right)
$$

so dass sich für das bestimmte Integral ergibt

$$
\frac{\mathrm{I}}{\sqrt{\mathrm{I}-h}} \lg \left(\frac{\sqrt{\mathrm{I}-h}}{h}+\sqrt{\frac{\mathrm{I}-h}{h^{2}}+\mathrm{I}}\right)=\lg \frac{\mathrm{I}}{h}+O(\mathrm{I})
$$

und daher $(47,7)$, w. z. b. w.

Lemma 5. Das Integral

$$
\int_{0}^{1} \int_{-\pi}^{\pi} \frac{r d \vartheta d r}{|I-z|}, \quad z=r e^{i \vartheta}
$$

konvergiert. - Dies folgt sofort aus dem Lemma 4, wenn man beide Seiten von $(47,7)$ mit $r$ multipliziert und nach $r$ von $o$ bis I integriert.

48. Beweis der Sätze XVII, XVIII; Anwendung des Spiegelungsprinzips. Es genügt, den Satz XVIII zu beweisen. Ohne Beschränkung der Allgemeinheit 
dürfen wir $\vartheta_{0}=0$ annehmen. Man bilde mit Hilfe der Randfunktion $\chi(\vartheta)$ von $V(z)$ auf $I$ das Integral

$(48, \mathrm{I}) \quad f^{*}(z)=\frac{i}{2 \pi} \int_{-\pi}^{\pi} \frac{e^{i \vartheta}+z}{e^{i \vartheta}-z} \chi(\vartheta) d \vartheta$

wobei $\chi(\vartheta)$ ausserhalb $\Gamma$ gleich o zu setzen ist. Dann besitzt der Imaginärteil der Funktion $f^{*}(z)$ in jedem Stetigkeitspunkt der Funktion $\chi(\boldsymbol{y})$, d. h. nach Voraussetzung in jedem von o verschiedenen Punkte $e^{i \vartheta}$ von $\Gamma$ den Randwert $\chi(\vartheta)$. Andererseits ist für den Realteil von $f^{*}(z)$ wegen der gleichmässigen Beschränktheit von $\chi(\vartheta)$ das $(47,3)$ entsprechende Integral beschränkt. Daraus folgt, wenn $f(z)-f^{*}(z)=f_{\mathrm{t}}(z)=U_{1}(z)+i V_{1}(z)$ gesetzt wird, dass einerseits das über das Innere von $\sigma$ erstreckte Integral

$$
\iint_{0}\left|U_{1}\left(r e^{i \vartheta}\right)\right| r d r d \vartheta=C<\infty
$$

ist und andererseits der Imaginärteil $V_{1}(z)$ in jedem von $z=\mathrm{I}$ verschiedenen Punkt des Bogens $T$ den Randwert o besitzt.

Es sei $\sigma^{\prime}$ das Spiegelbild von $\sigma$ in Bezug auf $E_{z}$. Das aus $\sigma, \sigma^{\prime}$ und $\boldsymbol{l}$ bestehende Gebiet sei mit $\sigma^{*}$ bezeichnet. Für $T \equiv E_{z}$ ist offenbar $\sigma^{*}$ mit der ganzen Riemannschen Kugel identisch. - Vermöge des Schwarzschen Spiegelungsprinzips lässt sich $f_{1}(z)$ in das Gebiet $\sigma^{*}$ fortsetzen, als eine in $\sigma^{*}$ bis eventuell auf $z=\mathrm{I}$ eindentige und reguläre Funktion, die in jedem Punkt $z^{\prime}=\frac{\mathrm{I}}{\bar{z}}$ von $\sigma^{\prime}$ für $|z|<1$ den Wert $\overline{f_{1}}(z)=U_{1}(z)-i V_{1}(z)$ besitzt. Führen wir aber im Integral $(48,2) r^{\prime}=\frac{I}{r}$ als neue Integrationsvariable ein, so verwandelt es sich in das über das $r^{\prime} e^{i g}$-Gebiet $\sigma^{\prime}$ erstreckte Integral

$(48,3)$

$$
\iint \frac{\left|U_{1}\left(\frac{1}{r^{\prime}} e^{i \vartheta}\right)\right|}{r^{\prime 4}} r^{\prime} d r^{\prime} d \vartheta .
$$

Hier ist nach dem Obigen $U_{1}\left(\frac{1}{r^{\prime}} e^{i 9}\right)=U_{1}\left(r^{\prime} e^{i 9}\right)$. Lassen wir nun im Integral $(48,3)$ die Striche bei $r^{\prime}$ weg, so ergibt sich, dass für unsere in das Innere von $\sigma^{*}$ bis auf $z=$ I fortgesetze Potentialfunktion $U_{1}(z)$ auch das Integral 
Über den Habitus der konformen Abbildung am Rande des Abbildungsbereiches. 159 $(48,4)$

$$
\iint_{\sigma^{\prime}} \frac{\left|U_{1}\left(r e^{i 9}\right)\right|}{r^{4}} r d r d \vartheta=C
$$

ist. Daraus folgt aber die Existenz zweier positiver Zahlen $\varrho, C_{1}$ derart, dass die Kreisscheibe $|z-\mathrm{I}| \leqq \frac{1}{\varrho}$ ganz in $\sigma^{*}$ liegt und

$$
\iint_{|z-1| \leqq \frac{1}{\varrho}}\left|U_{1}(z)\right| r d r d \vartheta \leqq C_{1}
$$

gilt.

Wir schicken nunmehr den Punkt $z=\mathrm{I}$ durch die Transformation $\zeta=\frac{\mathrm{I}}{z-\mathrm{I}}$ ins Unendliche. Dann geht $f_{1}(z)$ in $f_{1}\left(I+\frac{I}{\zeta}\right)=G(\zeta)$, eine für $o<\varrho \leqq|\zeta|<\infty$ eindeutige und reguläre Funktion von $\zeta$, über. Wir werden nun zuerst beweisen, dass $G(\zeta)$ im Unendlichen höchstens einen Pol zweiter Ordnung hat.

Denn setzt $\operatorname{man} G(\zeta)=U_{2}(\zeta)+i V_{2}(\zeta)$, wo $U_{2}(\zeta)=U_{1}\left(1+\frac{1}{\zeta}\right)$ ist, so folgt aus $(48,5)$, wegen $\frac{d z}{d \zeta}=\frac{-\mathrm{I}}{\zeta^{2}}$

$$
\int_{|z-1| \leqq \frac{1}{\rho}}\left|U_{1}(z)\right| r d r d \vartheta=\int_{|\zeta| \geqq 0} \int_{0}\left|U_{2}(\zeta)\right| \frac{\mathrm{I}}{|\zeta|^{4}} d \tau \leqq C_{1},
$$

wo $d \tau$ das Flächenelement in der $\zeta$-Ebene ist. Hieraus folgt aber für beliebig grosse positive $R \geqq 2 \varrho$

$$
\int_{\varrho \leqq 1 \zeta \mid \leqq R} \int_{\rho}\left|U_{2}(\zeta)\right| d \tau \leqq R^{4} \int_{\varrho \leqq|\zeta| \leqq R} \int_{\mid}\left|U_{2}(\zeta)\right| \frac{\mathrm{I}}{|\zeta|^{4}} d \tau \leqq R^{4} C_{1}
$$

Es sei nun $g(\zeta)$ der Hauptteil von $G(\zeta)$ in der Umgebung des unendlich fernen Punktes. $g(\zeta)$ ist auf jeden Fall eine ganze Funktion von $\zeta$, und es darf $g(0)=0$ vorausgesetzt werden. Setzt man nun $g(\zeta)=U^{*}(\zeta)+i V^{*}(\zeta)$, so folgt aus der obigen Ungleichung offenbar

$$
\int_{2 \varrho \leqq 1} \int_{i \leq R}\left|U^{*}(\zeta)\right| d \tau \leqq C_{2} R^{4}
$$


für eine positive Konstante $C_{2}$, da ja die Differenz $G(\zeta)-g(\zeta)$ für $|\zeta| \geqq 2 \varrho$ ihrem absoluten Betrage nach beschränkt bleibt.

Es sei nun $\varrho_{1}$ eine positive Zahl $\geqq 4 \varrho$. Drückt man den Wert der ganzen Funktion $g_{1}(u)=g\left(\varrho_{1} u\right)$ in den Punkten des Kreises $|u| \leqq \frac{\mathrm{I}}{2}$ durch die Werte ihres Realteiles $U^{*}\left(\varrho_{1} e^{i \vartheta}\right)$ auf der Kreislinie $|u|=1$ vermöge der Formel

$$
g\left(\varrho_{1} u\right)=g_{1}(u)=\frac{\mathrm{I}}{2 \pi} \int_{-\pi}^{\pi} \frac{e^{i g}+u}{e^{i g}-u} U^{*}\left(\varrho_{1} e^{i \vartheta}\right) d \vartheta
$$

aus, so gilt $\left|\frac{e^{i \vartheta}+u}{e^{i \vartheta}-u}\right| \leqq \frac{\mathrm{I}+\frac{\mathrm{I}}{2}}{\mathrm{I}-\frac{\mathrm{I}}{2}}=3$. Daher folgt für alle $\zeta$ mit $|\zeta| \geqq 2 \varrho$ und $2|\zeta| \leqq \varrho_{1}$

$$
|g(\zeta)| \leqq \frac{3}{2 \pi} \int_{-\pi}^{\pi}\left|U^{*}\left(\varrho_{1} e^{i \vartheta}\right)\right| d \vartheta
$$

Für ein beliebiges $\zeta$ mit $|\zeta|=r>2 \varrho$ integriere man die Relation $(48,8)$ nach $\varrho_{1}$ zwischen den Grenzen $2 r$ und $3 r$. Dann folgt

$$
r|g(\zeta)| \leqq \frac{3}{2 \pi} \int_{2 r}^{3 r} \int_{-\pi}^{\pi}\left|U^{*}\left(\varrho_{1} e^{i \vartheta}\right)\right| d \vartheta d \varrho_{1}
$$

Hier ist das Doppelintegral wegen $(48,6)\left(d \tau=\varrho_{1} d \vartheta d \varrho_{1}\right)$ höchstens gleich

$$
\frac{\mathrm{I}}{2 r} \int_{2}^{3 r} \int_{-\pi}^{\pi}\left|U^{*}\left(\varrho_{1} e^{i v}\right)\right| \varrho_{1} d \vartheta \cdot d \varrho_{1} \leqq \frac{\mathrm{I}}{2 r} C_{2}(3 r)^{4}=O\left(r^{3}\right)
$$

sodass also für $\zeta \rightarrow \infty g(\zeta)=O\left(|\zeta|^{2}\right)$ gilt und daher $g(\zeta)$ ein höchstens quadratisches Polynom in $\zeta$ ist, wie behauptet. Geștalt

49. Der Hauptteil von $f_{1}(z)$ für $z=\mathrm{I}$. Nach dem Obigen hat $f_{1}(z)$ die $(49,1)$

$$
f_{1}(z)=\frac{a}{(\mathrm{I}-z)^{2}}+\frac{b}{\mathrm{I}-z}+c+E(z), \quad E(\mathrm{I})=0,
$$


Über den Habitus der konformen Abbildung am Rande des Abbildungsbereiches. 161 wo $E(z)$ in $\sigma^{*}$ regulär ist. Nun folgt aus der vorausgesetzten Konvergenz des Integrals $(46,3)$ unter Berücksichtigung der Lemmata 3 und 5 ; dass das Integral $(49,2)$

$$
\int_{|z| \leqq 1}\left|\mathfrak{R} \frac{a}{(1-z)^{2}}\right| d \vartheta d r, \quad z=r e^{i \vartheta}
$$

auch konvergent sein muss. Es sei $a=|a| e^{i \alpha}$. Beachtet man, dass der Kreissektor $|z-\mathrm{I}|<\mathrm{I},|\arg (\mathrm{I}-z)|<\frac{\pi}{4}$ in $K_{z}$ enthalten ist, so folgt, I $-z=\varrho e^{i \varphi}$ gesetzt, dass das Integral

$$
\int_{0}^{1} \int_{-\frac{\pi}{4}}^{\frac{\pi}{4}} \frac{|a||\cos (\alpha-2 \varphi)|}{\varrho^{2}} d \varphi d \varrho=\int_{-\frac{\pi}{4}}^{\frac{\pi}{4}}|\cos (\alpha-2 \varphi)| d \varphi \int_{0}^{1} \frac{|a| d \varrho}{\varrho^{2}}
$$

konvergieren muss, was offenbar nur für $a=0$ möglich ist.

Damit nimmt $f_{1}(z)$ die Gestalt an

$$
\frac{b}{I-z}+c+E(z)
$$

sodass für $z=e^{i \vartheta}, b=i \varrho e^{i \beta}, \varrho \leqq 0,0 \leqq \beta<\pi$, wegen $\frac{\mathrm{I}}{\mathrm{I}-e^{i \bar{\vartheta}}}=\frac{i}{2} \frac{e^{-i_{\mathrm{i}}^{\vartheta}}}{\sin \frac{\vartheta}{2}}$,

$$
f_{1}\left(e^{i \vartheta}\right)=\frac{-\varrho e^{i\left(\beta-\frac{9}{2}\right)}}{2 \sin \frac{\vartheta}{2}}+c+E\left(e^{i \vartheta}\right)
$$

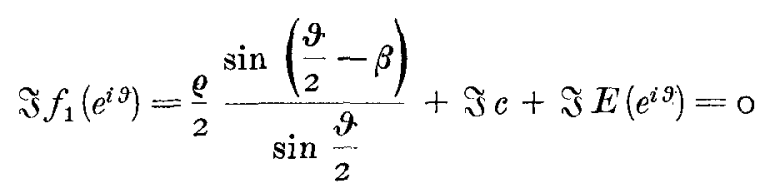

für $e^{i \vartheta}, \boldsymbol{\vartheta} \neq 0$, auf $\Gamma$ gilt. Da aber hier der Nenner des ersten Gliedes für $\boldsymbol{\vartheta} \rightarrow$ o in der ersten Ordnung verschwindet, muss auch der Zähler für $\vartheta=0$ verschwinden, und aus $\sin \beta=0$ folgt, wegen $0 \leqq \beta<\pi$, dass $\beta$ verschwindet, sodass $i b$ reell sein muss. Dann aber nimmt die linke Seite von $(49,3)$ den Wert $\frac{\varrho}{2}+\Im c+\Im E\left(e^{i \vartheta}\right)$ an, sodass, für $\vartheta \rightarrow$ o, wegen $E(\mathrm{I})=\mathrm{o}$,

21-34472. Acta mathematica. 64. Imprimé le 1 novembre 1934. 


$$
\Im c=-\frac{b}{2 i}, \quad \Im E\left(e^{i \vartheta}\right)=\text { o für } e^{i \vartheta} \text { auf } \Gamma
$$

ist. Für diesen Wert von $\Im c$ nimmt aber nunmehr $f_{1}(z)$, wenn $u=\frac{\varrho}{2}=\frac{b}{2 i}$, $v=\mathfrak{R} c$ gesetzt wird, die Gestalt an:

$$
f_{1}(z)=i u \frac{\mathrm{I}+z}{\mathrm{I}-z}+v+E(z), \quad E(\mathrm{I})=\mathrm{o}
$$

womit der Satz XVIII bewiesen ist.

\section{$\S$ I I. Erweiterungen des Randverzerrungssatzes.}

50. Bemerkungen über die gleichmässige Gültigkeit des Satzes IV. Unter welchen Umständen gelten nun die Formeln (I I, 2), ( I I, 3) gleichmässig für eine Randpunktmenge $M$ von $G_{1}$ ? Wir wollen $G_{1} \equiv K_{z}$ annehmen, so dass $\gamma_{1}=\pi$ ist. Greifen wir nun auf den Beweis des Satzes IV zurück, so sehen wir, dass der auf die Funktion $(\mathrm{I} 2,2)$ bezügliche Bestandteil der linken Seite von (I I, 2) sicher gleichmässig gegen $\frac{\gamma-\pi}{\pi}$ konvergiert, da $\gamma$ ja absolut beschränkt ist. Es kommt also nur darauf an, ob das Produkt $(1-|z|) g^{*}(z)$ gleichmässig gegen Null konvergiert, wenn $z$ im Winkel gegen die Punkte $z_{0}$ von $M$ strebt. Um nun $(7,5)$ anzuwenden, genügt es nicht, vorauszusetzen, dass der Realteil von $g^{*}(z)$ auf $E_{z}$ in den Punkten von $M$ gleichmässig stetig ist, was ja darauf hinausläuft, dass für $z_{0}$ auf der Menge $M$ die Funktionen

$$
\arg \frac{f(z)-f\left(z_{0}\right)}{z-z_{0}}
$$

gegen ihre rechts- bzw. linksseitigen Grenzwerte gleichmässig konvergieren, wenn $z$ jeweils längs $E_{z}$ gegen $z_{0}$ strebt. Es muss vielmehr ausserdem noch vorausgesetzt werden, dass die Funktionen $(50,1)$ in $K_{z}$ in ihrer Gesamtheit gleichmässig beschränkt sind. Dies wird aber sicher dann gewährleistet, wenn man das Gebiet $G^{*}$ der Nr. 13 so wählen kann, dass $\left|\varphi^{\prime}(z)\right|$ in allen Bildpunkten von $M$ zwischen festen positiven Schranken bleibt. Und dies ist nach dem in $\mathrm{Nr}$. I 3 Gesagten z. B. dann der Fall, wenn $\boldsymbol{M}$ ein abgeschlossener zusammenhängender Bogen von $E_{z}$ ist, dessen Bild ganz im Innern eines offenen Jordanschen Randbogens von $G$ liegt. Unter diesen Voraussetzungen aber gelten die Formeln $(\mathrm{I} I, 2)$ und $(\mathrm{I} I, 3)$ in der Tat gleichmässig, wenn $z$ jeweils gegen $z_{0}$ innerhalb 
Über den Habitus der konformen Abbildung am Rande des Abbildungsbereiches. 163 eines Winkels strebt, dessen Schenkel - zwei ron $z_{0}$ ausgehende Sehnen von $E_{z}$ - etwa feste positive Winkel mit der Tangente an $E_{z}$ in $z_{0}$ bilden.

51. Der. Fall einer Durchschnittstangente. Eine erste Verallgemeinerungsmöglichkeit des Randverzerrungssatzes wird durch die Tatsache nahe gelegt, dass die Relation $(7,4)$ nach Satz XIII ja bereits aus der Durchschnittsstetigkeit von $\chi(\vartheta)$, d. h. aus der Gültigkeit von $(36, \mathrm{I})$ gefolgert werden kann. Wird bei der Herleitung des Randverzerrungssatzes von dieser allgemeineren Tatsache Gebrauch gemacht, so kann man die Relationen (I I, 2) und (II,3), etwa $G_{1}=E_{z}$ gesetzt, beweisen, wenn

$$
\arg \frac{f\left(e^{i \vartheta}\right)-w_{0}}{z-\mathrm{I}}
$$

»im Durchschnitt» gegen $\frac{\pi}{2}-\frac{\gamma}{2}$ bzw. $-\left(\frac{\pi}{2}-\frac{\gamma}{2}\right)$ konvergiert, oder also, wenn die Richtungen der von $w_{0}$ ausgehenden Sehnen der Berandung von $G$ »im Durchschnitt» gegen gewisse Richtungen konvergieren, sofern ihre Schwankung bescbränkt bleibt. In diesem Falle ist also sogar die Existenz der Tangenten nicht wesentlich. Es ist indessen zu beachten, dass dabei von der Parameterdarstellung des Randes von $G$ Gebrauch zu machen ist, bei der der Parameter eben erst durch die konforme Abbildung festgelegt wird, so dass man eine solche Erweiterung des Randverzerrungssatzes erst dann als einen wirklichen Einblick in den Zusammenhang zwischen dem Verhalten von $f(z)$ und dem geometrischen Charakter der Berandung von $G$ in der Nähe von $w_{0}$ ansehen kann, wenn eine Formulierung für die Existenz einer "Durchschnittstangente» gegeben wird, die von der rein differentialgeometrisch zu charakterisierenden Parameterdarstellung abhängt. Für gewisse spezielle Zwecke mag dagegen diese Erweiterung von Interesse sein.

52. Spezialisierung von Satz $X V I$ für $n=\mathrm{I}$. Dagegen ist eine andere Verschärfung unserer Sätze von Bedeutung, in der der Begriff der Tangente durch denjenigen der Grenzstützen ersetzt wird.

Wir wollen zuerst den Satz XVI für $n=\mathrm{I}$ und $z_{0}=e^{i \vartheta_{0}}$ spezialisieren und etwas umformen. Wegen $(39,8)$ ist

$$
\begin{aligned}
(52, \mathrm{I}) \quad K_{1}(\psi)=\cos \psi+\operatorname{tg} \psi \int_{-\psi}^{\frac{\pi}{2}} \cos x d x=\cos \psi+\operatorname{tg} \psi(\mathrm{I}+\sin \psi) & = \\
& =\frac{\cos \psi}{\mathrm{I}-\sin \psi}=\frac{\mathrm{I}+\sin \psi}{\cos \psi} .
\end{aligned}
$$


Andererseits liefert die Formel (10,6), wenn in ihr $z$ durch $z e^{-i \vartheta_{0}}=\frac{z}{z_{0}}$ ersetzt wird, $(52,2)$

$$
\mathrm{I}-|z|=\left(\mathrm{I}-z e^{-i \vartheta_{0}}\right) e^{i \psi} \cos \psi=\left(z_{0}-z\right) e^{-i \vartheta_{0}} e^{i \psi} \cos \psi
$$

Tragen wir $(52,1)$ und $(52,2)$ in die Formel $(43,4)$ des Satzes XVI ein, so ergibt sich

$$
\begin{aligned}
& \overline{\operatorname{Lin}}\left\{\left|\pi\left(z_{0}-z\right) e^{i \psi} \cos \psi f^{\prime}(z)+i \Delta\left(\vartheta_{0}\right) e^{i \psi} \cos \psi\right|-\right. \\
&\left.-\frac{2}{\cos \psi}\left[k_{+}(\mathrm{I}+\sin \psi)+k_{-}(\mathrm{I}-\sin \psi)\right]\right\} \leqq 0
\end{aligned}
$$

oder aber (über die Bedeutung von $\psi$ vgl. Nr. 48)

$(52,3) \quad\left(z_{0}-z\right) f^{\prime}(z)=\frac{-i \Delta\left(9_{0}\right)}{\pi}+\frac{2 \Theta}{\pi}\left(\frac{k_{+}}{\mathrm{I}-\sin \psi}+\frac{k_{-}}{\mathrm{I}+\sin \psi}\right)+\varepsilon\left(z-z_{0}\right)$

wo $\varepsilon\left(z-z_{0}\right)$ gegen $\circ$ strebt, wenn $z$ gegen $z_{0}$ im Winkel konvergiert, und $\Theta$ eine Grösse ist, deren absoluter Betrag I nicht überschreitet.

53. Die verallgemeinerten Voraussetzungen. Es sei nun $P$ ein einfacher Randpunkt eines endlichen einfach zusammenhängenden Gebietes $G$ in der $v$ Ebene, und der Rand von $G$ möge in der Nähe von $P$ eine Jordankurve bilden. Entspricht $P=w_{0}$ bei der konformen Abbildung auf das Innere von $E_{z}$ dem Punkt $z=z_{0}=e^{i \vartheta_{0}}$, so sind damit die beiden Randbogen $C_{-}, C_{+}$, die in $P$ zusammenstossen, gewissen Halbumgebungen von $z=z_{0}$ auf $E_{z}$ mit $z=e^{i 9}$, $\vartheta<\vartheta_{0}$ bzw. $\vartheta>\vartheta_{0}$ zugeordnet, so dass man bei positivem Umlauf um das Innere von $G$ von $C_{-}$auf $C_{+}$übertritt. Es mögen nun die Bogen $C_{-}, C_{+}$im Punkte $w_{0}$ Grenzstützen besitzen, die in den Winkeln $\left|\arg \left(w-w_{0}\right)-h_{-}\right| \leqq k_{-}$ bzw. $\left|\arg \left(w-w_{0}\right)-h_{+}\right| \leqq k_{+}$mit endlichen $h_{-}, h_{+}, k_{-}, k_{+}$liegen. Dabei sollen die Winkelbestimmungen insbesondere so gewählt werden, dass sie auch längs eines in der Umgebung von $w_{0}$ liegenden, $C_{-}$mit $C_{+}$verbindenden Querschnittes von $G$ stetig bleiben. Sind insbesondere $h_{-} \pm k_{-}, h_{+} \pm k_{+}$die Winkel der Richtungen der vier Grenzstützen selbst mit der positiven reellen Axe, so ist $h_{+}-h_{-}=-\gamma$ eine negative Zahl; denn die beiden Grenzstützen an $C_{-}$werden in die entsprechend gelegenen Grenzstützen an $C_{+}$durch Drehung um $w_{0}$ in negativer Richtung übergeführt (nämlich durch das Innere von $G$ ), und daher bildet die Winkelhalbierende der beiden Grenzstützen an $C_{+}$einen kleineren Winkel mit der positiven reellen Axe als die Winkelhalbierende der beiden Grenzstützen an $C_{\ldots}$. Im allgemeineren Fall kann aber $\gamma=h_{-}-h_{+}$auch o oder 
Über den Habitus der konformen Abbildung am Rande des Abbildungsbereiches. 165 negativ werden. Endlich soll $\left|\arg \left(w-w_{0}\right)\right|$ bei stetiger Fortsetzung unterhalb einer festen Schranke bleiben, wenn $w$ den ganzen Rand von $G$ durchläuft.

54. Anwendung des Satzes XVI. Strebt nun $\vartheta$ monoton wachsend gegen $\vartheta_{0}$, so folgt

$$
h_{-}-k_{-} \leqq \tilde{\operatorname{Lim}} \arg \left(f\left(e^{i 9}\right)-w_{0}\right) \leqq h_{-}+k_{-},
$$

und da dabei $\arg \left(e^{i \vartheta}-z_{0}\right) \rightarrow \vartheta_{0}-\frac{\pi}{2}$ gilt, folgt

$$
\left|\overline{\operatorname{Lim}} \arg \frac{f(z)-w_{0}}{z-z_{0}}-h_{-}+\vartheta_{0}-\frac{\pi}{2}\right| \leqq k_{-}, z=e^{i \vartheta}, \vartheta \uparrow \vartheta_{0}
$$

Da anderseits für $\vartheta \downarrow \vartheta_{0} \arg \left(e^{i \vartheta}-z_{0}\right) \rightarrow \vartheta_{0}-3 \frac{\pi}{2}$ ist, folgt analog

$$
\left|\overline{\operatorname{Lim}} \arg \frac{f(z)-w_{0}}{z-z_{0}}-h_{+}+\vartheta_{0}-3 \frac{\pi}{2}\right| \leqq k_{+}, z=e^{i \vartheta}, \vartheta \downarrow \vartheta_{0}
$$

Daher ist für $g_{+}=h_{+}-\vartheta_{0}+3 \frac{\pi}{2}, g_{-}=h_{-}-\vartheta_{0}+\frac{\pi}{2}, d=h_{+}-h_{-}+\pi$ die Formel $(52,3)$ auf die Funktion

$$
\frac{1}{i} g(z)=\frac{\mathbf{I}}{i} \lg \frac{f(z)-w_{0}}{z-z_{0}}
$$

anwendbar, und wir erhalten

$$
\begin{aligned}
\frac{\mathrm{I}}{i}\left(z_{0}-z\right) g^{\prime}(z)=-i \frac{h_{+}-h_{-}+\pi}{\pi} & + \\
& +\frac{2 \Theta}{\pi}\left(\frac{k_{+}}{\mathrm{I}-\sin \psi}+\frac{k_{-}}{\mathrm{I}+\sin \psi}\right)+\varepsilon\left(z-z_{0}\right),
\end{aligned}
$$

oder für $h_{-}-h_{+}=\gamma$

$$
(54,5) \frac{f^{\prime}(z)}{\frac{f(z)-w_{0}}{z-z_{0}}}=\mathrm{I}+\left(z-z_{0}\right) g^{\prime}(z)=\frac{\gamma}{\pi}+\frac{2 \Theta_{0}}{\pi}\left(\frac{k_{+}}{1-\sin \psi}+\frac{k_{-}}{1+\sin \psi}\right)+\varepsilon_{1}\left(z-z_{0}\right),
$$

und diese Relation gilt gleichmässig auf einem $\vartheta_{0}$-Bogen $M$, auf dem die Voraussetzungen über $\arg \left(w-w_{0}\right)$ gleichmässig gelten und die Grössen $h_{-}, h_{+}$, $k_{-}, k_{+}$gleichmässig beschränkt sind, sofern dabei $|\psi| \leqq \frac{\pi}{2}-\delta, \delta>$ o bleibt. - 
Schalten wir nun, genau wie in Nr. 13 beim Beweis des Satzes IV ein Gebiet $G^{*}$ dazwischen, so ergibt sich endlich, dass die Voraussetzung der Beschränktheit von $\left|\arg \left(w-w_{0}\right)\right|$ für alle Randpunkte $w$ von $G$ unwesentlich ist. -

Es seien nun z. B. längs eines $\vartheta_{0}$-Bogens $M$ auf $E_{z}$ gleichmässig die Relationen $(54,2)$ und $(54,3)$ erfüllt mit festen, von $\vartheta_{0}$ unabhängigen Werten von $k_{+}$und $k_{-}$und mit gleichmässig beschränkten $h_{+}$und $h_{-}$, für die durchweg längs $M h_{-}-h_{+}=\pi$ gilt; ist dann $M_{1}$ ein abgeschlossener, ganz im Innern von $M$ liegender Bogen, so folgt aus $(54,5)$ für $\psi=0$ die Relation

$$
\overline{\operatorname{Lim}}\left|\frac{f^{\prime}(z)}{\frac{f(z)-f\left(z_{0}\right)}{z-z_{0}}-\mathrm{x}}\right| \leqq 2 \frac{k_{+}+k_{-}}{\pi},
$$

gleichmässig, wenn $z$ radial gegen die Punkte $z_{0}$ von $M_{1}$ strebt.

55. Der verallgemeinerte Randverzerrungssatz. Wir fassen unsere obigen Ergebnisse im folgenden Satz zusammen:

Satz XIX: Es bestehe der Rand eines einfach zusammenhängenden Gebietes $G$ der w-Ebene in der Nähe eines Randpunktes $P=w_{0}$ aus zwei Jordanbogen $C_{-}, C_{+}$, wobei man in $w_{0}$ beim positiven Umlauf um das Innere von $G$ von $C_{-}$auf $C_{+}$übertritt. Die Randbogen $C_{-}, C_{+}$mögen in $P$ Grenzstützen besitzen, die in den Winkelräumen

$$
\left|\arg \left(w-w_{0}\right)-h_{-}\right| \leqq k_{-} \text {bzw. }\left|\arg \left(w-w_{0}\right)-h_{+}\right| \leqq k_{+}
$$

mit endlichen $h_{-}, h_{+}, k_{-}, k_{+}$liegen, wenn die Winkelbestimmungen längs eines in der Umgebung von $w_{0}$ liegenden Querschnitts von $G$ stetig bleiben. Bildet dann $w=f(z)$ das Innere von $K_{z}$ konform auf das Innere von $G$ ab, und entspricht dabei $z_{0}=e^{i 9_{0}}$ dem Punkte $w_{0}$, so gilt, wenn $z$ aus dem Innern von $K_{z}$ im Winkel gegen $z_{0}$ strebt,

$$
\begin{aligned}
\left(z-z_{0}\right) g^{\prime}(z)=\frac{\gamma}{\pi}-\mathrm{I} & +\frac{2 \Theta}{\pi}\left(\frac{k_{+}}{\mathrm{I}-\sin \psi}+\frac{k_{-}}{\mathrm{I}+\sin \psi}\right)+ \\
& +\varepsilon\left(z-z_{0}\right), g(z)=\lg \frac{f(z)-w_{0},}{z-z_{0}},
\end{aligned}
$$

$$
\frac{f^{\prime}(z)}{\frac{f(z)-w_{0}}{z-z_{0}}}=\frac{\gamma}{\pi}+\frac{2 \Theta}{\pi}\left(\frac{k_{+}}{\mathrm{I}-\sin \psi}+\frac{k_{-}}{\mathrm{I}+\sin \psi}\right)+\varepsilon\left(z-z_{0}\right)
$$


Über den Habitus der konformen Abbildung am Rande des Abbildungsbereiches. 167 wo $\gamma=h_{-}-h_{+}, \psi=-\arg \left(\mathrm{I}-z e^{-i 9_{0}}\right),|\Theta| \leqq \mathrm{I}$ ist und $\varepsilon\left(z-z_{0}\right)$ gegen o konvergiert, wenn $z$ so gegen $z_{0}$ strebt, dass dabei $|\psi| \leqq \frac{\pi}{2}-\delta, \delta>0$ bleibt.

Zusate zum Satz XIX: Sind für feste $k_{+}, k_{-}$die Voraussetzungen dieses Satzes längs eines freien abgeschlossenen Jordanbogens $C$ des Randes von $G$ gleichmässig erfüllt, sind dabei $h_{+}, h_{-}$gleichmässig beschränkt und gilt durchweg $h_{-}-h_{+}=\pi$, so gilt, wenn $M_{1}$ der Bildbogen von $C$ ist, gleichmässig auf $M_{1}$

$$
\overline{\operatorname{Lim}}\left|\frac{f^{\prime}(z)}{\frac{f(z)-f\left(z_{0}\right)}{z-z_{0}}}-\mathrm{I}\right| \leqq 2 \frac{k_{+}+k_{-}}{\pi},
$$

wenn $z$ aus dem Innern von $K_{z}$ radial gegen $z_{0}$ auf $M_{1}$ strebt.

Endlich folgt unter den Voraussetzungen des Satzes XIX für

$$
\gamma>2(k+k)
$$

nach $(55,3)$ aus dem Bestehen einer der beiden Relationen

$$
f^{\prime}(z) \rightarrow \infty, \frac{f(z)-w_{0}}{z-z_{0}} \rightarrow \infty
$$

das Bestehen der anderen, solange $\psi$ auf einen Bereich

$$
\frac{2 k_{+}}{\mathrm{I}-\sin \psi}+\frac{2 k_{-}}{\mathrm{I}+\sin \psi} \leqq \gamma-\delta, \delta>0
$$

beschränkt bleibt. Besitzt aber für $(55,5) f^{\prime}(z)$ oder $\frac{f(z)-w_{0}}{z-z_{0}}$ bei Konvergenz im Winkel einen Grenzwert oder endlichen Limsup. bzw. Liminf., so folgen aus $(55,3)$ entsprechende Schranken für Limsup. bzw. Liminf. des anderen Ausdrucks, solange $\psi$ auf einen Bereich $(55,7)$ beschränkt bleibt. - Und genau das Gleiche gilt offenbar für die drei Relationenpaare

$$
f^{\prime}(z) \rightarrow 0, \frac{f(z)-w_{0}}{z-z_{0}} \rightarrow 0
$$

$$
\overline{\operatorname{Lim}}\left|f^{\prime}(z)\right|<\infty, \overline{\operatorname{Lim}}\left|\frac{f(z)-w_{0}}{z-z_{0}}\right|<\infty ;
$$

$$
\underline{\operatorname{Lim}}\left|f^{\prime \prime}(z)\right|>0, \quad \underline{\operatorname{Lim}}\left|\frac{f(z)-w_{0}}{z-z_{0}}\right|>0 \text {. }
$$


56. Der Fall eines allgemeinen Gebietes $G_{1}$. Wir haben im Satze XIX die Abbildung eines Gebietes $G$ auf das Innere von $E_{z}$ betrachtet, anstatt, wie im Satze IV, auch das Gebiet $G_{1}$ allgemein zu wählen. Man kann nun in einem gewissen Umfang auch den Satz XIX auf den Fall ausdehnen, dass das zugrunde gelegte Gebiet in der $z$-Ebene nicht das Innere von $E_{z}$ ist, sondern ein Gebiet $G_{1}$ von ähnlicher Beschaffenheit wie $G$. Sind dann die entsprechenden Konstanten für das Gebiet $G_{1}$ etwa $\gamma_{1}>0, k_{+}^{\prime}, k_{-}^{\prime}$, so kann man die Abbildung von $G_{1}$ auf $G$ durch $V$ Vermittelung» der Abbildung auf das Innere von $E_{5}$ in der $\zeta$-Ebene ausführen. Wird der Winkel $\psi$ auf die $\zeta$-Ebene bezogen, so ergibt sich durch Division der beiden $(54,5)$ entsprechenden Relationen

$$
\frac{f^{\prime}(z)}{\frac{f(z)-w_{0}}{z-z_{0}}}=\frac{\gamma+2 \Theta\left(\frac{k_{+}}{\mathrm{I}-\sin \psi}+\frac{k-}{\mathrm{I}+\sin \psi}\right)+\varepsilon\left(w-w_{0}\right)}{\gamma_{1}+2 \Theta_{1}\left(\frac{k_{+}^{\prime}}{\mathrm{I}-\sin \psi}+\frac{k_{-}^{\prime}}{\mathrm{I}+\sin \psi}\right)+\varepsilon_{1}\left(z-z_{0}\right)},
$$

wo $|\Theta| \leqq I,\left|\Theta_{1}\right| \leqq I$ ist. Ist nun $\gamma_{1}>2\left(k_{+}^{\prime}+k_{-}^{\prime}\right)$, so kann man für die Werte von $\psi$, für die

$$
\gamma_{1}>\frac{2 k_{+}}{\mathrm{I}-\sin \psi}+\frac{2 k_{-}}{\mathrm{I}+\sin \psi}+\delta, \delta>0
$$

ist, die obige Relation auf die Form bringen:

$$
\frac{f^{\prime}(z)}{\frac{f(z)-w_{0}}{z-z_{0}}}=\frac{\gamma+2 \Theta\left(\frac{k_{+}}{\mathrm{I}-\sin \psi}+\frac{k_{-}}{\mathrm{I}+\sin \psi}\right)}{\gamma_{1}+2 \Theta_{1}\left(\frac{k_{+}^{\prime}}{\mathrm{I}-\sin \psi}+\frac{k_{-}^{\prime}}{\mathrm{I}+\sin \psi}\right)}+\varepsilon\left(z-z_{0}\right)
$$

Die Hauptschwierigkeit bei der Anwendung dieser Formel besteht darin, dass der Winkel $\psi$ ja nicht in der $z$-sondern in der $\zeta$-Ebene gemessen werden muss. Nun besteht, wie wir bald zeigen werden, auch im Falle, wenn $k_{+}^{\prime}$ und $k_{-}^{\prime}$ nicht verschwinden, noch eine gewisse angenäherte Winkelproportionalität bei der Abbildung von $G_{1}$ auf das Innere von $E_{\zeta}$, die allerdings um so weniger ausgesprochen ist, je grösser die Werte von $k_{+}^{\prime}, k_{-}^{\prime}$ sind. Immerhin lassen sich wenigstens im Falle relativ kleiner $k_{+}^{\prime}, k_{-}^{\prime}$ Schranken für $\psi$ aus den Werten des entsprechenden Winkels in der $z$-Ebene ermitteln, mit deren Hilfe dann der Anwendungsbereich der Formel $(56,3)$ abgegrenzt werden kann. 
Über den Habitus der konformen Abbildung am Rande des Abbildungsbereiches. 169

57. Der Fall eines Gebiets $G_{1}$ mit einer Ecke in $z_{0}$. In dem Falle aber, wo $k_{+}^{\prime}=k_{-}^{\prime}=\mathrm{o}$ ist, wo also $G_{1}$ im Punkte $z_{0}$ eine Ecke der Oeffinung $\gamma_{1}>0$ bildet, ergibt sich aus der Formel $(56,3)$ ohne Weiteres

$$
\frac{f^{\prime}(z)}{\frac{f(z)-w_{0}}{z-z_{0}}}=\frac{\gamma}{\gamma_{1}}+\frac{2 \Theta}{\gamma_{1}}\left(\frac{k_{+}}{\mathrm{I}-\sin \psi}+\frac{k-}{\mathrm{I}+\sin \psi}\right)+\varepsilon\left(z-z_{0}\right)
$$

solange $|\psi| \leqq \frac{\pi}{2}-\delta$ ist. Hierbei ist allerdings unter $\psi$ noch der in der $\zeta$-Ebene zu messende Winkel zu verstehen. Nun wird aber (vgl. Nr. I5) bei der konformen Abbildung von $G_{1}$ auf das Innere von $E_{\zeta}$ jeder innerhalb $G_{1}$ gelegene Winkel mit dem Scheitelpunkt in $z_{0}$ beim Übergang zur $\zeta$-Ebene im Verhältnis $\frac{\pi}{\gamma_{1}}$ vergrössert - es besteht die sogenannte Winkelproportionalität bei der Eckenabbildung - und es wird die ins Innere von $G_{1}$ weisende Richtung der Winkelhalbierenden des Eckenwinkels von $G_{1}$ bei $z_{0}$ in die radiale Richtung beim entsprechenden Punkt $\zeta_{0}$ auf $E_{\xi}$ übergeführt. Versteht man daher unter $\psi$ nunmehr den Winkel des Vektors $z-z_{0}$ mit der »inneren» Winkelhalbierenden des Eckenwinkels von $G_{1}$ bei $z_{0}$, so muss in der zuletzt hergeleiteten Formel $\psi$ durch $\frac{\pi}{\gamma_{1}} \psi$ ersetzt werden, und $\psi$ ist nunmehr der Bedingung zu unterwerfen $|\psi| \leqq \frac{\gamma_{1}}{2}-\delta, \delta>0$

Wir formulieren das Ergebnis als

Satz XX: Wird das Gebiet $G$ des Satzes XIX in der w-Ebene auf das Gebiet $G_{1}$ des Satzes IV in der $z$-Ebene mit Hilfe der Funktion $w=f(z)$ konform abgebildet und versteht man unter $\psi$ den Winkel, den die Richtung von $z_{0}$ nach $z$ mit der inneren Winkelhalbierenden des Eckenwinkels von $G_{1}$ bei $z_{0}$ bildet, so gilt

$$
(57,2) \quad \frac{f^{\prime}(z)}{\frac{f(z)-w_{0}}{z-z_{0}}}=\frac{\gamma}{\gamma_{1}}+\frac{2 \Theta}{\gamma_{1}}\left(\frac{k_{+}}{1-\sin \frac{\pi \psi}{\gamma_{1}}}+\frac{k-}{\mathrm{I}+\sin \frac{\pi \psi}{\gamma_{1}}}\right)+\varepsilon\left(z-z_{0}\right), \quad|\Theta| \leqq \mathrm{I}
$$

wo $\varepsilon\left(z-z_{0}\right)$ gegen o konvergiert, wenn $z$ so aus dem Innern von $G_{1}$ im Winkel gegen $z_{0}$ strebt, dass $|\psi| \leqq \frac{\gamma_{1}}{2}-\delta, \delta>0$ bleibt.

22-34472. Acta mathematica. 64. Imprimé le 1 novembre 1934. 
58. Gleichmässige und allseitige Stetigkeit der Drehung am Rande. Aus dem Beweis des Satzes $\nabla$ ergibt sich, wenn $G_{1} \equiv K_{z}$ angenommen wird, dass die Formeln $(\mathrm{I} 4, \mathrm{I}),(\mathrm{I} 4,2),(\mathrm{I} 4,3)$ gleichmässig für alle Punkte $z_{0}$ eines Bogens $\boldsymbol{M}$ von $E_{z}$ gelten, wie er in $\mathrm{Nr}$. 50 charakterisiert wurde, wenn dabei $z$ gegen $z_{0}$ in der dort gekennzeichneten Weise konvergiert. Die Konstante $c$ ist dabei natürlich von $z_{0}$ abhängig.

Daraus folgt insbesondere für $\gamma=\pi$, wenn der Rand $G$ in der Umgebung von $w_{0}$ eine durchweg stetige Tangente besitzt, dass die Gleichung $(14,2)$ für die Punkte eines gewissen Bogens ron $E_{z}$ um $z_{0}$, bei radialer Approximation etwa, gleichmässig gilt. Die Konstante $c$ ist dabei gleich dem Winkel zwischen den innern Normalen in den einander entsprechenden Randpunkten von $E_{z}$ und $G$ und ist daher stetig auf einem Bogen von $E_{z}$ um $z_{0}$. Daraus folgt, dass $($ I 4,2$)$ für einen geeigneten derartigen Bogen um $z_{0}$ und insbesondere für den Punkt $z_{0}$ selbst auch bei allseitiger Annäherung aus dem Innern von $E_{z}$ richtig bleibt.

Durch zweimalige Anwendung dieses Resultats ergibt sich ferner: Haben unter den Voraussetzungen des Satzes IV die Ränder von $G_{1}$ und $G$ in den Punkten $z_{0}, w_{0}$ stetige Tangenten, so dass insbesondere $\gamma=\gamma_{1}=\pi$ ist, so bleibt die Formel $(\mathrm{I} 4,2)$ auch richtig, wenn $z$ aus dem Innern von $G_{1}$ allseitig gegen $z_{0}$ konvergiert. Dies ist ein Spezialfall des Lindelöfschen Satzes (Satz VI).

59. Das Analogon von $(14,3)$ unter den Voraussetzungen des Satzes XIX. Wir legen nunmehr die Voraussetzungen des Satzes XIX zugrunde. Um dann in der Formel $(55,3)$ dieses Satzes die Argumente rechts und links vergleichen zu können, müssen wir voraussetzen, dass

$$
\gamma>2\left(k_{+}+k_{-}\right)
$$

ist. Dann gibt es positive $\delta$, für die die Menge der $\psi$ mit

$$
\gamma \geqq \frac{2 k+}{\mathrm{I}-\sin \psi}+\frac{2 k-}{\mathrm{I}+\sin \psi}+\delta, \delta>0
$$

nicht leer ist. Für entsprechende $z$ gilt dann (bei geeigneter Argumentfestlegung)

$$
\arg f^{\prime}(z)-\arg \frac{f(z)-w_{0}}{z-z_{0}}=\arg \left(I+\frac{2 \Theta}{\gamma}\left(\frac{k_{+}}{\mathrm{I}-\sin \psi}+\frac{k_{-}}{\mathrm{I}+\sin \psi}\right)\right)+\varepsilon_{1}\left(z-z_{0}\right) .
$$

Nun gilt für $|\zeta|<$ I

$$
\arg (1+\zeta) \leqq \arcsin |\zeta|
$$


Über den Habitus der konformen Abbildung am Rande des Abbildungsbereiches. 171 (dies ergibt sich sofort, wenn man vom Nullpunkt aus eine Tangente an den Kreis um den Punkt I mit dem Radius $|\zeta|$ legt). Hieraus folgt weiter

$(59,3)\left|\arg f^{\prime}(z)-\arg \frac{f(z)-w_{0}}{z-z_{0}}\right| \leqq \arcsin \left(\frac{2}{\gamma}\left(\frac{k_{+}}{I-\sin \psi}+\frac{k_{-}}{\mathrm{I}+\sin \psi}\right)\right)+\varepsilon\left(z-z_{0}\right)$ wo $\varepsilon\left(z-z_{0}\right)$ gegen o konvergiert, wenn $z$ so nach $z_{0}$ strebt, dass dabei $\psi=-\arg \left(\mathrm{I}-\frac{z}{z_{0}}\right)$ im Bereich $(59,2)$ bleibt. Hier ist der arcus sinus auf jeden Fall höchstens gleich

$$
\arcsin \left(\mathrm{I}-\frac{\delta}{\gamma}\right)
$$

Die Formel $(59,3)$ gilt also jedenfalls, wenn $z$ gegen $z_{0}$ innerhalb eines hinreichend schmalen Winkels um den in $z_{0}$ mündenden Radius konvergiert.

Ferner gitt die Formel $(59,3)$ gleichmässig, d. h. $\varepsilon\left(z-z_{0}\right)$ konvergiert gegen o gleichmässig für einen $z_{0}$-Bogen $M_{1}$ auf $E_{z}$, der den Voraussetzungen des Zusatzes zum Satz XIX genügt, wenn fïr ein festes $\psi$-Intervall die Relationen $(59,2)$ mit festem $\delta>0$ erfüllt sind, und $z$ gegen $z_{0}$ jeweils innerhalb des entsprechenden $\psi$-Winkels konvergiert.

6o. Das Analogon von (I4, I) unter den Voraussetzungen des Satzes XIX. Andererseits gelten unter den Voraussetzungen des Satzes XIX die Formeln $(54,2)$ und $(54,3)$. Beachtet man nun, dass für jedes $z_{0}=e^{i 9_{0}}$

$$
\psi=\psi\left(z_{0}, z\right)=-\arg \left(\mathrm{I}-\frac{z}{z_{0}}\right)=-\Im \lg \left(\mathrm{I}-\frac{z}{z_{0}}\right)
$$

eine innerhalb und auf $E_{z}$ beschränkte Potentialfunktion von $z$ ist, die auf $E_{z}$ für $\vartheta \downarrow \vartheta_{0}$ bzw. $\vartheta \uparrow \vartheta_{0}$ die Grenzwerte $\frac{\pi}{2}$ bzw. $-\frac{\pi}{2}$ besitzt, so ergeben sich für die Funktion

$(60,2) \quad P(z)=\arg \frac{f(z)-w_{0}}{z-z_{0}}-\frac{h_{+}+h_{-}}{2}-\pi+\vartheta_{0}-\frac{\pi-\gamma}{\pi} \psi\left(z_{0}, z\right), \gamma=h_{-}-h_{+}$,

die Relationen

$(60,3) \quad \overline{\operatorname{Lim}}_{g \downarrow 9_{0}}|P(z)| \leqq k_{+} \quad$ bzw. $\quad \overline{\operatorname{Lim}}_{g \uparrow 9_{0}}|P(z)| \leqq k_{-}$ 
Aus $(60,3)$ folgt aber weiter, dass die Potentialfunktion

$$
P_{1}(z)=P(z)-\frac{k_{+}+k_{-}}{2}-\frac{k_{+}-k_{-}}{\pi} \psi\left(z_{0}, z\right)
$$

nach oben beschränkt ist und für $\vartheta \rightarrow \vartheta_{0}$ auf $E_{z}$ einen nicht positiven Limes superior besitzt. Ist daher $\varphi_{1}(z)$ eine innerhalb $E_{z}$ reguläre Funktion mit dem Realteil $P_{1}(z)$, so bleibt $e^{\varphi_{1}(z)}$ im Innern von $E_{z}$ beschränkt, und die Randwerte von $\left|e^{p_{1}(z)}\right|$ auf $E_{z}$ haben für $\boldsymbol{\vartheta} \rightarrow \vartheta_{0}$ einen Limes superior, der $\leqq$ I ist. Daher gilt, wenn $z$ aus dem Innern von $E_{z}$ allseitig gegen $z_{0}$ konvergiert, $\overline{\operatorname{Lim}}\left|e^{\varphi_{1}(z)}\right| \leqq \mathrm{I}$, und daher

$$
\overline{\operatorname{Lim}}\left(P(z)-\frac{k_{+}+k_{-}}{2}-\frac{k_{+}-k_{-}}{\pi} \psi\left(z_{0}, z\right)\right) \leqq 0
$$

Genau ebenso ergibt sich

$$
\operatorname{Lim}\left(P(z)+\frac{k_{+}+k_{-}}{2}+\frac{k_{+}-k_{-}}{\pi} \psi\left(z_{0}, z\right)\right) \geqq 0 .
$$

Dies bedeutet aber, dass man $P(z)$ in der Form schreiben kann

$$
P(z)=\Theta\left(\frac{k_{+}+k_{-}}{2}+\frac{k_{+}-k_{-}}{\pi} \psi\left(z_{0}, z\right)\right)+\varepsilon\left(z-z_{0}\right)
$$

wo $|\Theta| \leqq \mathrm{I}$ ist und $\varepsilon\left(z-z_{0}\right)$ gegen o konvergiert, wenn $z$ aus dem Innern von $E_{z}$ allseitig gegen $z_{0}$ strebt. Da andererseits nach Definition von

$$
\psi\left(z_{0}, z\right)=-\arg \left(1-\frac{z}{z_{0}}\right)=\arg z_{0}-\arg \left(z_{0}-z\right)
$$

wegen $\arg \left(z-z_{0}\right)-\arg \left(z_{0}-z\right)=-\pi^{32}, \arg z_{0}=\vartheta_{0}$,

$$
\psi\left(z_{0}, z\right)=\vartheta_{0}-\pi-\arg \left(z-z_{0}\right)
$$

gilt, folgt aus $(60,2)$, dass unter den Voraussetzungen des Satzes XIX

${ }^{82}$ Man beachte, dass diese Formel dann richtig ist, wenn $\arg \left(z_{0}-z\right)$, $\arg \left(z-z_{0}\right)$ so festgelegt werden, dass $\arg \left(z_{0}-z\right)$ im Intervall $\left(-\frac{\pi}{2}+\vartheta_{0}, \frac{\pi}{2}+\vartheta_{0}\right)$ und $\arg \left(z-z_{0}\right)$ im Intervall $\left(-\frac{3 \pi}{2}+\vartheta_{0},-\frac{\pi}{2}+\vartheta_{0}\right)$ bleiben. Offenbar sind bei diesen Festlegungen unsere Argumentfunktionen für alle $z$ aus $K_{z}$ stetig. 
Über den Habitus der konformen Abbildung am Rande des Abbildungsbereiches. 173 $(60,6) \quad \arg \frac{f(z)-w_{0}}{z-z_{0}}=\left(\frac{\gamma}{\pi}-\mathrm{I}\right) \arg \left(z-z_{0}\right)+c+P(z)$

ist, wo $c=\frac{3 h_{-}-h_{+}}{2}-\frac{\gamma}{\pi} \vartheta_{0}$ ist und für $P(z)$ die Relation $(60,4)$ gilt. Und die Relation $(60,4)$ gilt hier gleichmässig für einen $z_{0}$-Bogen $M_{1}$ auf $E_{z}$, wenn für ihn die Voraussetzungen des Zusatzes zum Satze XIX erfüllt sind und die Relationen $(54,2)$ und $(54,3)$ gleichmässig gelten.

Es sei noch hervorgehoben, dass bei der Herleitung der Formeln $(60,4)$, $(60,6)$, weder von der Annahme $(59, I)$ noch überhaupt von der Annahme, dass $\gamma>0$ ist, Gebrauch gemacht worden. ${ }^{33}$

61. Herleitung des Lindelöfschen Satzes. Damit sind unter den Voraussetzungen des Satzes XIX die zu (I4, I) und (I4,3) analogen Formeln aufgestellt, aus denen offensichtlich sich ohne weiteres die zu $(\mathrm{I} 4,2)$ analoge Formel ergibt. Wesentlich ist an diesen Formeln, dass die von $k_{+}$und $k_{-}$abhängigen Gliederaggregate etwa für $\psi=0$ gegen o konvergieren, wenn dies für $k_{+}$und $k_{-}$der Fall ist. Daraus folgt, ähnlich wie in Nr. 58, dass, wenn der Rand von G unter den Voraussetzungen des Satzes XIX im Punkte $z_{0}$ eine L-Tangente besitzt, die Formel $(14,2)$ für allseitig gegen $z_{0}$ konvergierende $z$ gilt - der Lindelöfsche Satz. -

62. Grössenordnung von $f(z)$ und $f^{\prime}(z)$.

Satz XXI. Unter den Voraussetzungen von Satz IV gilt, wenn $z$ gegen $z_{0}$ im Winkel konvergiert,

$$
\frac{\lg \left|f(z)-w_{0}\right|}{\lg \left|z-z_{0}\right|} \rightarrow \frac{\gamma}{\gamma_{1}}, \gamma \geqq 0
$$

und fiir $\gamma>0$

$$
\frac{\lg \left|f^{\prime}(z)\right|}{\lg \left|z-z_{0}\right|} \rightarrow \frac{\gamma}{\gamma_{1}}-\mathrm{I}, \gamma>0
$$

Fïr $\gamma=$ o aber gilt

$$
\overline{\operatorname{Lim}} \frac{\lg \left|f^{\prime}(z)\right|}{\lg \frac{\mathrm{I}}{\left|z-z_{0}\right|}} \leqq \mathrm{I}
$$

33 Das Hauptresultat $(60,4),(60,6)$ dieser Nummer findet sich im Prinzip bei W. Gross (1) pp. $273-279$. 
$(62,4)$

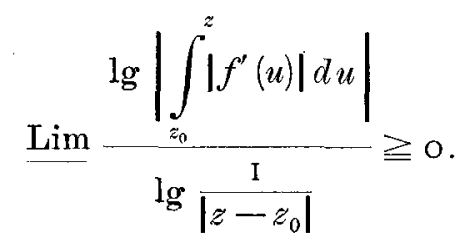

Beweis: Beim Beweis von $(62, \mathrm{I})$ genügt es, $G_{1} \equiv K_{z}, \gamma_{1}=\pi$ anzunehmen, da, wegen $\gamma_{1}>0,(62$, I) sich durch Division der beiden analogen Formeln ergibt, die sich auf die Abbildung von $G$ und $G_{1}$ auf das Innere des Einheitskreises beziehen. Dann aber folgt $(62,1)$ unmittelbar aus dem Satz XXI ${ }^{\circ}$, der weiter unten in dieser Nummer bewiesen wird.

$(62,2)$ ergibt sich für $\gamma>0$, wenn man die Formel (I I, 3) logarithmiert:

$$
\lg \left|f^{\prime}(z)\right|-\lg \left|f(z)-w_{0}\right|+\lg \left|z-z_{0}\right|-\lg \frac{\gamma}{\gamma_{1}} \rightarrow 0
$$

durch $\lg \left|z-z_{0}\right|$ dividiert und $(62,1)$ berücksichtigt:

$$
\frac{\lg \left|f^{\prime}(z)\right|}{\lg \left|z-z_{0}\right|}-\frac{\gamma}{\gamma_{1}}+\mathrm{I} \rightarrow \mathrm{O}
$$

Für $\gamma=\mathrm{o}$ aber folgt aus $(\mathrm{I}, \mathrm{I}, 3)$

$$
\lg \left|f^{\prime}(z)\right|-\lg \left|f(z)-w_{0}\right|+\lg \left|z-z_{0}\right| \rightarrow-\infty
$$

Dividieren wir aber die linke Seite durch $\lg \frac{1}{\left|z-z_{0}\right|}$, so bleibt auf jeden Fall der $\overline{\operatorname{Lim}}$ der linken Seite nicht positiv. Wegen $(62,1)$ folgt dann $(62,3)$.

Um aber $(62,4)$ zu beweisen, gehe man von der Formel

$$
f(z)-w_{0}=\int_{z_{0}}^{\tilde{z}} f^{\prime}(u) d u
$$

mit geradlinigem Integrationsweg aus und beachte, dass, wegen $(62,1)$, für $\gamma=0$

$$
\left|f(z)-w_{0}\right| \geqq\left|z-z_{0}\right|^{\varepsilon}
$$

für beliebig kleine positive $\varepsilon$ gilt, sobald $z$, in einer festen Winkelumgebung von $z_{0}$ in $G_{1}$ bleibend, nahe genug an $z_{0}$ herankommt. Daher liefert $(62,5)$ für hinreichend kleine $\left|z-z_{0}\right|$ : 
Über den Habitus der konformen Abbildung am Rande des Abbildungsbereiches. 175

$$
\begin{gathered}
\left|z-z_{0}\right|^{\varepsilon} \leqq\left|\int_{z_{0}}^{z}\right| f^{\prime}(u)|d u|, \\
\frac{\lg \left|\int_{z_{0}}^{z}\right| f^{\prime}(u)|d u|}{\lg \frac{1}{\left|z-z_{0}\right|}} \geqq-\varepsilon,
\end{gathered}
$$

woraus, da $\varepsilon$ beliebig klein angenommen worden kann, $(62,4)$ ohne weiteres folgt.

Aus $(62,1)$ folgen die am Schlusse der Nr. 30 angegebenen Tatsachen über $(30,1)$ und $(30,2)$ ohne weiteres.

Satz $X X I^{\circ}$. Unter den Voraussetzungen des Satzes XIX gilt, wenn z gegen $z_{0}$ im Winkel konvergiert,

$$
\overline{\operatorname{Lim}}\left|\frac{\lg \left|f(z)-w_{0}\right|}{\lg \left|z-z_{0}\right|}-\frac{\gamma}{\pi}\right| \leqq \frac{k_{+}+k_{-}}{\pi} .
$$

Zum Beweis wende man auf die Funktion $\frac{\mathrm{I}}{i} \lg \left(f(z)-w_{0}\right)$ den Satz XVI an. Für diese Funktion ist $A=-\gamma$. Dann ergibt sich aus $(43,3)$

$$
\widetilde{\operatorname{Lim}}\left|\frac{\pi \lg \left|f(z)-w_{0}\right|}{-i \lg \left|\mathrm{I}-\frac{z}{z_{0}}\right|}-i \gamma\right| \leqq k_{+}+k_{-},
$$

woraus die Behauptung des Satzes unmittelbar folgt. ${ }^{34}$

\section{$\S$ I2. Ergänzungssätze zum Randrerzerrungssatz für Spitzenabbildungen.}

63. Allgemeine Charakterisierung der Resultate. Während der Randverzerrungssatz auch für $\gamma=0$ gilt, lassen sich aus ihm in diesem Falle nicht die gleichen Folgerungen ziehen, wie sie für $\gamma>0$ in den $\$ \$ 5,6$ und I I gezogen

${ }^{34}$ Die Relation $(62, \mathrm{I})$ findet sich für $G_{1}=K_{z}$ in W. Gross (2) p. 6I, wo sich zugleich (p. 60) auch der Satz $\mathrm{XXI}^{\circ}$ findet, mit etwas anderen Bezeichnungen, aber sogar unter etwas allgemeineren Annahmen über den Rand von $G$. Für den Spezialfall $G_{1}=K_{z}, \gamma=\pi$ ist $(62, \mathrm{I}) \mathrm{mit}$ dem Satz 3 in VISSER (2) p. 3 I aequivalent. 
worden sind. Dennoch bleiben verschiedene der oben hergeleiteten Tatsachen auch für $\gamma=0$ richtig, zum Teil unter geeigneten einschränkenden Voraussetzungen.

Dies gilt erstens für die Formel (I4, I), die ja in der Nr. I4 auch für $\gamma=0$ bewiesen wird. Ebenso bleibt auch der im $\$ 5$ hergeleitete Satz über den Quotienten $\frac{f\left(z_{2}\right)-w_{0}}{f\left(z_{1}\right)-w_{0}}$ bei Approximation im Winkel, für $\gamma=0<\gamma_{1}$ spezialisiert, richtig, wie in $\mathrm{Nr} .64$ bewiesen werden wird.

Wird ferner vorausgesetzt, dass einer der in der Spitze zusammenstossenden Randzweige von $G$ dort eine $L$-Tangente hat, ebenso wie der entsprechende Randzweig in $G_{1}$, so gilt der Satz über den Quotienten $\frac{f\left(z_{2}\right)-w_{0}}{f\left(z_{1}\right)-w_{0}}$ auch bei $h a l b$ seitiger Konvergenz gegen $z_{0}$ an jenem Randzweig von $G_{1}$ (Nr. 64, Satz XXII).

Wird aber endlich vorausgesetzt, dass die beiden in der Spitze zusammenstossenden Randzweige von $G$, ebenso wie die entsprechenden Randzweige von $G_{1}$ in $w_{0}$ bzw. $z_{0} L$-Tangenten besitzen, so bleibt nicht nur der Satz über den Quotienten $\frac{f\left(z_{0}\right)-w_{0}}{f\left(z_{1}\right)-w_{0}}$ bei allseitiger Annäherung an $z_{0}$ richtig, sondern es gelten auch die Formeln $(14,2)$, (14,3) bei allseitiger Approximation (Nr. 65-67), und es bleibt dann ferner auch der im $\$ 5$ hergeleitete Satz über den Quotienten $\frac{f^{\prime}\left(z_{2}\right)}{f^{\prime}\left(z_{1}\right)}$ bei Approximation im Winkel, sowie die Formel $(62,2)$ gültig.

64. Relative Schwankung der absoluten Verzerrung am Rande. Satz XXII. Es mögen die Voraussetzungen des Satzes VIII gelten, es sei aber jetzt $\mathrm{o}=\gamma<\gamma_{1}$. Dann gilt, wenn $z_{1}$ und $z_{2}$ über eine Winkelumgebung von $z_{0}$ in $G_{1}$ derart nach $z_{0}$ streben, dass dabei für feste positive $c_{1}, c_{2}$

$$
o<c_{1} \leqq \frac{\left|z_{1}-z_{0}\right|}{\left|z_{2}-z_{0}\right|} \leqq c_{2}<\infty
$$

bleibt,

$$
\frac{f\left(z_{1}\right)-f\left(z_{0}\right)}{f\left(z_{2}\right)-f\left(z_{0}\right)} \rightarrow \mathrm{I}, \frac{f\left(z_{2}\right)-f\left(z_{1}\right)}{f\left(z_{2}\right)-f\left(z_{0}\right)} \rightarrow \text { o. }
$$

Wird ferner vorausgesetzt, dass einer der beiden in $w_{0}$ zusammenstossenden Randzweige von $G$ - er sei mit $C$ bezeichnet - in $w_{0}$ eine L-Tangente besitzt, ebenso wie der entsprechende Randzweig $C_{1}$ von $G_{1}$, so bleibt die Formel $(64,2)$ richtig, wenn $z_{1}, z_{2}$ gegen $z_{0}$ halbseitig am Randstück $C_{1}$ streben. - Besitzen endlich die 
Über den Habitus der konformen Abbildung am Rande des Abbildungsbereiches. 177 beiden in $w_{0}$ zusammenstossenden Randzweige von $G$ in $w_{0} L$-Tangenten, ebenso wie die entsprechenden Randzweige von $G_{1}$ in $z_{0}$, so bleibt $(64,2)$ richtig, wenn $z_{1}, z_{2}$ gegen $z_{0}$ aus $G_{1}$ allseitig konvergieren.

Beweis. Es darf ohne Beschränkung der Allgemeinheit $G_{1}=K_{z}, z_{0}=\mathbf{I}$ vorausgesetzt werden. In der Tat kann ja die Abbildung von $G_{1}$ auf $G$ auf dem Umwege über $K_{z}$ ausgeführt werden, wobei die Bedingung $(64, \mathrm{I})$ wegen der Aussage $c$ ) des Satzes VIII in eine analoge Bedingung übergeht.

Man betrachte nun die Funktion $h(z)=\frac{\mathrm{I}}{i} \lg \left(f(z)-w_{0}\right)$, deren Realteil in $G_{1}$ irgendwie normiert sein möge. Aus der Formel $(14,1)$, die ja auch für $\gamma=0$ in

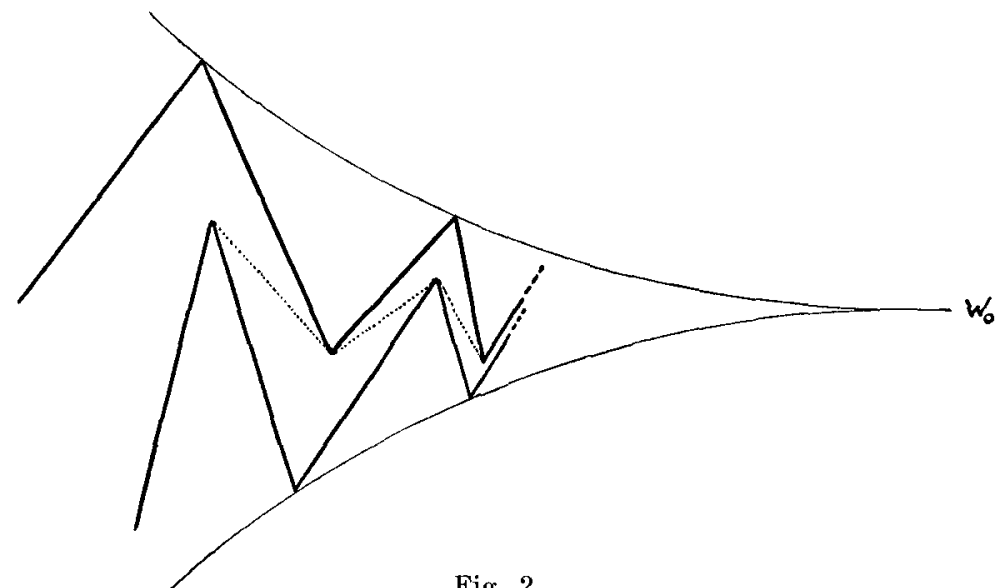

Fig. 2 .

Nr. I4 bewiesen wurde, folgt, dass $\Re h(z)$ in der Umgebung von $z=\mathrm{I}$ stetig ist. Wendet man daher auf die Funktion $h(z)$ den Zusatz 2 zum Satz XVI mit $\vartheta_{0}=0$ an, so folgt aus $(45,6)$

$$
h\left(z_{1}\right)-h\left(z_{2}\right) \rightarrow o
$$

womit die erste Formel $(64,2)$ bewiesen ist. Die zweite Formel folgt daraus unmittelbar.

Wird aber vorausgesetzt, dass die Randzweige $C, C_{1} L$-Tangenten besitzen, so verfahren wir ganz analog wie in Nr. I9. Ohne Beschränkung der Allgemeinheit darf vorausgesetzt werden, dass erstens $z_{0}=0$ und das Gebiet $G_{1}$ mit dem Kreissektor identisch ist, der aus dem Einheitskreis $K_{z}$ durch die beiden unter dem Winkel $\frac{\pi}{4}$ zur negativen reellen Axe geneigten Radien herausgeschnit23-34472. Acta mathematica. 64. Imprimé le 31 octobre 1934. 
ten wird, zweitens, dass $w_{0}=0$ ist und das Randstück $C$ von $G$ mit einer der beiden Teilstrecken der reellen Axe in der $w$-Ebene identisch ist, die zwischen $w_{0}=0$ und $w_{0}=\mathrm{I}$ bzw. $w_{0}=\mathrm{o}$ und $w_{0}=-\mathrm{I}$ liegen, ferner, dass $G$ vollständig in der oberen Hälfte des Einheitskreises $K_{w}$ der $w$-Ebene enthalten ist. Endlich darf angenommen werden, dass das Bild $C_{1}$ der Strecke $C$ einer der beiden Begrenzungsradien des Kreisselktors $G_{1}$ ist.

Nunmehr spiegele man wieder die Gebiete $G$ bzw. $G_{1}$ an den Strecken $C$ bzw. $C_{1}$ und wende auf die dadurch entstandenen "verdoppelten» Gebiete die Relation $(64,2)$ an. Sie gilt nunmehr in einem Winkel um $C_{1}$ mit der Spitze in $z_{0}$ woraus unsere Behauptung ohne weiteres folgt. Damit ist zugleich auch die letzte Behauptung von Satz XXII und daher der ganze Satz XXII bewiesen.

65. Ein Gegenbeispiel. Inwiefern kann man erwarten, dass die Formeln $(14,2)$ und $(14,3)$ für $\gamma=o$, d. h. im Falle einer Spitze richtig bleiben?

Ein Blick auf die Figur 2 lehrt, dass die Gültigkeit von (14,2) und damit auch von $(\mathrm{I} 4,3) \mathrm{im}$ allgemeinen Fall nicht zu erwarten ist. In der Tat würde aus $(14,2)$, auch nur für radiale Konvergenz von $z$ gegen $z_{0}$, folgen, dass der nach $z_{0}$ führende Radius von $E_{z}$ auf eine Kurve abgebildet wird, für die die Tangentenrichtung sich stetig der Tangentenrichtung im Punkte $w_{0}$ anschliesst. Nun bildet der in der Figur 2 zwischen den beiden Zickzacklinien eingeschlossene Bereich im Punkte $w_{0}$ eine Spitze, denn die beiden Zickzacklinien besitzen offenbar in $w_{0}$ eine gemeinsame Tangente. Andererseits kann man die Breiten der einzelnen Teilstïcke unseres Zickzackbereiches zu gegebenen Neigungen der Teilstrecken des Randes so wählen, dass innerhalb dieses Bereichs keine einfache Kurve bis $w_{0}$ verlaufen kann, deren Tangentenrichtung sich an die Tangentenrichtung im Punkte $w_{0}$ stetig anschliesst.

Man braucht hierzu offenbar nur zu verlangen, dass es in beliebiger Nähe des Punktes $w_{0}$ Sehnen geben muss, deren Richtungen von der Richtung der Spitzentangente um einen festen positiven Winkel abweichen. Hierzu braucht man aber nur dafür zu sorgen, dass die Winkel, die die in der Figur punktiert gezeichneten Verbindungsstrecken der aufeinanderfolgenden Eckpunkte der einspringenden Ecken mit der Spitzentangente bilden, absolut genommen, oberhalb einer festen positiven Zahl bleiben. -

Es sind daher ergänzende Voraussetzungen nötig, um die Gültigkeit der Formeln $(\mathrm{I} 4,2),(\mathrm{I} 4,3)$ auch im Falle $\gamma=0, \gamma_{1}>0$ sicherzustellen. Eine solche Bedingung ist nun, wie wir zeigen werden, die, dass die beiden in $w_{0}$ zusammen- 
Über den Habitus der konformen Abbildung am Rande des Abbildungsbereiches. 179

stossenden Randstücke im Punkte $w_{0}$ L-Tangenten besitzen, - dass also, wie wir sagen werden, $G$ in $w_{0}$ eine L-Tangentenspitze besitzt. Und zwar gelten dann unsere Formeln, wenn $z$ aus dem Innern von $G_{1}$ allseitig gegen $z_{0}$ konvergiert, sobald auch die beiden Randzweige von $G_{1}$ in $z_{0} L$-Tangenten haben.

Beim. Beweis werden wir vom Satz XVII Gebrauch zu machen haben.

66. Vorbereitende Transformation von $G$. Um den Satz XVII anwenden zu können, müssen wir zuerst den Bereich $G$ des Satzes IV mit einer L-Tangentenspitze in $w_{0}$ geeignet transformieren. Ohne Beschränkung der Allgemeinheit darf $G_{1}=K_{z}, z_{0}=\mathrm{I}$ vorausgesetzt werden. Nun verbinden wir die beiden in $w_{0}$ zusammenstossenden Randstücke in der Nähe von $w_{0}$ durch einen Querschnitt des Gebietes $G$ und betrachten das durch diesen Querschnitt abgeschnittene, die Spitze enthaltende Teilstück $G^{\prime}$ von $G$. Bekanntlich lässt sich die Abbildungsfunktion $f_{1}(z)$ von $G^{\prime}$ auf $K_{z}$, die $z_{0}=\mathrm{I}$ in $w_{0}$ überführt, in der Form darstellen $f(\varphi(z))$, wo $f(z)$ die Abbildungsfunktion des Satzes IV ist und $\varphi(z)$ im Punkte $z=$ I regulär ist und dort eine von o verschiedene Ableitung besitzt. (Vgl. die in Nr. I 3 über das Gebiet $G^{*}$ angestellten Überlegungen.) Daher strebt $\arg \varphi^{\prime}(z)$ einem festen Grenzwert zu, wenn $z$ in $K_{z}$ allseitig gegen $z_{0}=$ I konvergiert, und man darf sich auf die Betrachtung von $G^{\prime}$ beschränken. Man verlängere nun das eine der beiden in $w_{0}$ zusammenstossenden Randstücke $C_{1}, C_{2}$ von $G^{\prime}$, etwa $C_{1}$, längs der Spitzentangente und bilde ein von einer Jordankurve begrenztes Gebiet $G^{\prime \prime}$, das $G^{\prime}$ ebenso wie die von $w_{0}$ verschiedenen Punkte von $C_{2}$ im Innern enthält und zu dessen Berandung sowohl $C_{1}$ als auch ein Stück der verlängerten Spitzentangente gehört. Dann hat der Rand von $G^{\prime \prime}$ im Punkte $w_{0}$ eine $L$-Tangente, so dass nach dem Lindelöfschen Satz (Satz VI) bei der Abbildung von $G^{\prime \prime}$ auf die Halbebene $\mathfrak{\Im} u>0$, wobei $w_{0}$ in den Nullpunkt übergeht, das Argument der Abbildungsfunktion einen festen Grenzwert besitzt, wenn $w$ gegen $w_{0}$ strebt. Bei dieser Abbildung geht das Gebiet $G^{\prime}$ in ein Gebiet $G^{(3)}$ mit der Spitze in $u=0$ über, wobei das eine der in der Spitze zusammenstossenden Randstücke ein Stück der reellen $u$-Axe ist, während das andere - wir bezeichnen es mit $C_{3}$ - wiederum eine $L$-Tangente in $u=0$ besitzt (vgl. Nr. I 7 ). Wir können also nunmehr $G$ durch $G^{(3)}$ ersetzen.

Wiederholen wir denselben Prozess mit $G^{(3)}$ und $C_{3}$, so geht $G^{(3)}$ in ein Gebiet $G^{(4)}$ etwa in der $v$-Ebene über, das eine Spitze etwa im Punkte $v=\mathrm{o}$ besitzt, wobei das eine der beiden in $v=0$ zusammenstossenden Randstücke ein Stück etwa der negativen reellen Axe ist, das andere aber in der Nähe von $u=0$ 
analytisch ist und im Punkte $u=\mathrm{o}$ selbst eine $L$-Tangente besitzt. Daher kann man $G$ durch $G^{(4)}$ ersetzen.

Endlich können wir vom Gebiet $G^{(4)}$ mit Hilfe eines geeigneten geradlinigen Querschnitts ein Teilgebiet abgrenzen und sodann die Ecken durch Kreisbogen "abrunden". So gelangen wir zu einem Gebiet $G^{(5)}$ in $\operatorname{der} t$-Ebene mit der Spitze in $t=t_{0}=0$.

Nun unterscheidet sich bei jedem einzelnen Schritt unserer Reduktion das Ableitungsargument der Abbildungsfunktion des alten Gebietes auf $K_{z}$ vom Ableitungsargument der Abbildungsfunktion des neuen Gebietes auf $K_{z}$ nur um eine Grösse, die einem festen Grenzwert zustrebt, wenn $z$ in $K_{z}$ allseitig gegen $z_{0}=\mathrm{I}$ konvergiert. Ist daher die Formel $(14,2)$ für die Abbildung des Gebietes $G$ zu beweisen, so genügt es, den Beweis für das Gebiet $G^{(5)}$ zu führen.

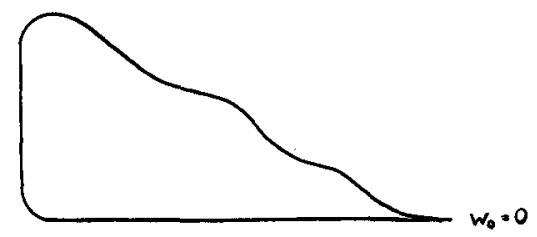

Fig. 3.

67. $\arg f^{\prime}(z)$ bei der Abbildung einer L-Tangentenspitze. Beim Beweis der Formel $(14,2)$ dürfen wir annehmen, das $G_{1} \equiv K_{z}$, und $\gamma=\pi$ ist. Ferner darf nach dem in Nr. 66 Gesagten angenommen werden, dass das Gebiet $G$ von vornherein die Eigenschaften des Gebietes $G^{(5)}$ und daher insbesondere die in der Figur 3 gezeichnete Gestalt besitzt. Insbesondere sei $w_{0}=0$ vorausgesetzt. Bildet nun $w=f(z)$ das Innere von $G$ so auf $K_{z}$ ab, dass dabei $w=0$ in $z=1$ übergeht, so muss in jedem von $z=\mathrm{I}$ verschiedenen Punkt von $E_{z} \arg f^{\prime}(z)$ stetig sein, da der Rand von $G$ überall bis auf $v=0$ eine stetige Tangente besitzt. Ferner ist der Gesamtzuwachs, den die Tangentenrichtung an $G$ erfährt, wenn man von der Spitze aus den Rand in positiver Richtung durchläuft, gleich $\pi$. Normieren wir nun $\arg f^{\prime}(z)$ so, dass es auf der oberen Hälfte von $E_{z}$ in der Umgebung von $z=\mathrm{I}$ sich wenig von $\frac{\pi}{2}$ unterscheidet, und beachten, dass der Gesamtzuwachs $\pi$ der Tangentenrichtung längs des Randes von $G$ gleich ist dem Gesamtzuwachs $2 \pi$ der Tangentenrichtung an $E_{z}$ plus dem Gasamtzuwachs von $\arg f^{\prime}(z)$, so sehen wir, dass für unsere Bestimmung von $\arg f^{\prime}(z)$

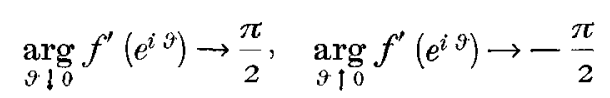


Über den Habitus der konformen Abbildung am Rande des Abbildungsbereiches. 181 gilt. Und aus der Stetigkeit von $\arg f^{\prime}\left(e^{i \vartheta}\right)$ für $0<\boldsymbol{\vartheta}<2 \pi$ folgt, dass $\left|\arg f^{\prime}\left(e^{i \vartheta}\right)\right|$ gleichmässig beschränkt ist. Um nun dasselbe für $\left|\arg f^{\prime}(z)\right|$ in $K_{z}$ zu beweisen, wenden wir auf die Funktion $\lg f^{\prime}(z)$ den Satz XVII an. Denn, dass die Voraussetzungen dieses Satzes für $\mathfrak{J} \lg f^{\prime}(z)$ erfüllt sind, haben wir soeben bewiesen. Da andererseits der Rand von $G$ rektifizierbar ist, ist bekanntlich

$$
\int_{0}^{2 \pi}\left|f^{\prime}\left(\gamma \cdot e^{i \vartheta}\right)\right| d \vartheta
$$

gleichmässig in $r$ für $o \leqq r<$ I beschränkt ${ }^{35}$. Daraus folgt aber bekanntlich, dass auch das Integral

$$
\int_{0}^{2 \pi}|\lg | f^{\prime}\left(r e^{i \vartheta}\right) \| d \vartheta
$$

gleichmässig in $r$ beschränkt ist ${ }^{36}$, woraus ohne weiteres folgt, dass auch die auf den Realteil von $\lg f^{\prime}(z)$ bezügliche Voraussetzung des Satzes XVII erfüllt ist. Daher gilt

$$
\lg f^{\prime}(z)=i u \frac{\mathrm{I}+z}{\mathrm{I}-z}+v+\frac{i}{2 \pi} \int_{-\pi}^{\pi} \frac{e^{i \vartheta}+z}{e^{i \vartheta}-z} \arg f^{\prime}\left(e^{i \vartheta}\right) d \vartheta
$$

mit reellen $u$ und $v$. Nun gelten für die in $K_{z}$ schlichte Funktion $f(z)$ die Ungleichungen des Verzerrungssatzes ${ }^{37}$

$$
\frac{\mathrm{I}-|z|}{(1+|z|)^{2}} \leqq\left|f^{\prime}(z)\right| \leqq \frac{\mathrm{I}+|z|}{(\mathrm{I}-|z|)^{2}}
$$

sowie die Ungleichung des Drehungssatzes ${ }^{38}$

${ }^{35} \mathrm{Vgl}$. BTEBERBACH (I), pp. I82-I 83 .

${ }^{30} \mathrm{Vgl}$. Ostrowski (I) u. (2). Aus der Beschränktheit von (a) folgt zuerst nur die Beschränktheit von $\int_{0}^{2 \pi} \lg \left|f^{\prime}\left(r e^{i \vartheta}\right)\right| d \vartheta$, wo allgemein $+\stackrel{+}{\lg } b=\lg b$ für $b \geq I$ und $\stackrel{+}{\lg } b=0$ für $b<\mathrm{I}$ ist. Dass aber dies mit der Beschränktheit von (b) äquivalent ist, habe ich in (I), p. 82 angegeben und in (2) p. 26I Fussnote ausgeführt.

37 Vgl. z. B. BIEBERBACH (2), p. 77.

${ }^{38}$ Vgl. z. B. BieberbACH (2), p. 8 I. 


$$
\left|\arg f^{\prime}(z)\right| \leqq 2 \lg \frac{1+|z|}{1-|z|}
$$

Daraus folgt aber, wenn $z$ im Winkel gegen I strebt,

$$
\lg f^{\prime}(z)=O\left(\lg \frac{\mathbf{I}}{\mathrm{I}-|z|}\right)
$$

Andererseits aber folgt, wegen der Beschränktheit von $\arg f^{\prime}\left(e^{i \vartheta}\right)$ aus $(43,3)$, Satz XVI,

$$
\int_{-\pi}^{\pi} \frac{e^{i \vartheta}+z}{e^{i \vartheta}-z} \arg f^{\prime}\left(e^{i \vartheta}\right) d \vartheta=O\left(\lg \frac{\mathrm{I}}{\mathrm{I}-|z|}\right)
$$

Daher ergibt sich aus $(67, \mathrm{I})$

$$
i u \frac{\mathrm{I}+z}{\mathrm{I}-z}=o\left(\lg \frac{\mathrm{I}}{\mathrm{I}--z}\right)
$$

sodass $u=0$ ist. Damit ergibt sich aus $(67, \mathrm{I})$

$$
\arg f^{\prime}(z)=\frac{\mathrm{I}}{2 \pi} \int_{-\pi}^{\pi} \frac{\left(\mathrm{I}-r^{2}\right) \arg f^{\prime}\left(e^{i \vartheta}\right)}{\mathrm{I}-2 r \cos (\vartheta-\varphi)+r^{2}} d \vartheta, z=r e^{i \varphi}
$$

sodass $\left|\arg f^{\prime}(z)\right|$ in $K_{z}$ beschrünkt ist. Nunmehr hat man nach dem bekannten Schema zu verfahren. Für die Potentialfunktion $\psi(z)=-\arg (1-z)$ des Satzes II hat die in $K_{z}$ beschränkte Potentialfunktion $\arg f^{\prime}(z)-\psi$ auf $E_{z}$ beschränkte und bis auf $z=-$ I stetige Randwerte, wobei der Randwert für $z=$ I verschwindet. Daraus folgt aber bekanntlich für $z \rightarrow \mathrm{I}$ bei allseitiger Konvergenz aus $K_{z}$

$$
\arg f^{\prime}(z)=\psi+o(\mathrm{I})=-\arg (z-\mathrm{I})-\pi+o(\mathrm{I})
$$

womit $(\mathrm{I} 4,2)$ (und damit wegen (I4, I) auch (I4,3)) bewiesen ist.

68. Das Verzerrungsverhältnis im Falle von L-Tangentenspitzen. Unter der gleichen Zusatzvoraussetzung, unter der oben die Formel $(\mathbf{1 4}, 2)$ für Spitzenabbildung hergeleitet wurde, unter der Annahme nämlich, dass die in den Punkten $z_{0}$ und $w_{0}$ zusammenstossenden Randzweige von $G_{1}$ und $G L$-Tangenten in diesen Punkten besitzen, lässt sich auch der Satz XXII über das absolute Verzerrungs- 
Über den Habitus der konformen Abbildung am Rande der Abbildungsbereiches. 183 verhältnis für $\gamma=0$ etwas verschärfen. Zunächst gilt bei $L$-Tangentenspitzen nach Satz XXII die Formel

$$
\frac{f\left(z_{1}\right)-w_{0}}{f\left(z_{2}\right)-w_{0}} \rightarrow \mathrm{I}
$$

unter der Annahme $(64, \mathrm{I})$, wenn $z$ aus dem Innern von $G_{1}$ allseitig gegen $z_{0}$ konvergiert.

Darüber hinaus gelten aber nunmehr auch die Relationen

$$
\frac{f^{\prime}\left(z_{1}\right)}{f^{\prime}\left(z_{2}\right)} \sim\left(\frac{z_{2}-z_{0}}{z_{1}-z_{0}}\right)^{\frac{\pi}{\gamma_{1}}}
$$

$$
\frac{\lg \left|f^{\prime}(z)\right|}{\lg \left|z-z_{0}\right|} \rightarrow-\frac{\gamma_{1}}{\pi},
$$

wenn für feste positive $e_{1}, c_{2}$

$$
c_{1} \leqq\left|\frac{z_{1}-z_{0}}{z_{2}-z_{0}}\right| \leqq c_{2}
$$

gilt und $z, z_{1}, z_{2}$ im Winkel aus $G_{1}$ gegen $z_{0}$ streben.

Beim Beweis von $(68,2)$ und $(68,3)$ darf nach $(21,2)$ und $(62,1)$ vorausgesetzt werden, dass $G_{1} \equiv K_{z}$ und $z_{0}=\mathrm{I}$ ist. Man betrachte nun die Funktion

$$
h(z)=\frac{\mathrm{I}}{i} \lg \left(\left(z-z_{0}\right) f^{\prime}(z)\right)
$$

Dann lässt sich das oben bewiesene Resultat über die Gültigkeit von (I4,2) für $\gamma=0$ bei allseitiger Konvergenz von $z$ gegen $z_{0}=\mathrm{I}$ aus dem Innern von $E_{z}$ so formulieren, dass dann

$$
\mathfrak{R} h(z) \rightarrow c
$$

gilt. Daraus folgt aber, dass die Randfunktion von $\Re h(z)$ in einer Umgebung von $z_{0}=\mathrm{I}$ in $K_{z}$ beschränkt ist, und daher in der Umgebung von $z_{0}$ auf $E_{z}$ nach Fatou bis auf eine Nullmenge eine Randwertfunktion besitzt, die in $z_{0}$ stetig ist. Daher genügt die Funktion $h(z)$ der Voraussetzung des Zusatzes 3 zum Satze XVI, wobei die Punktmenge $M$ dieses Satzes aus dem einen Punkt $z_{0}=\mathrm{I}$ besteht. Dann liefert der Zusatz 3 zum Satz XVI unmittelbar, wegen $(45,61)$, 
184

Alexander Ostrowski.

$$
\lg \left|\left(z_{1}-z_{0}\right) f^{\prime}\left(z_{1}\right)\right|-\lg \left|\left(z_{2}-z_{0}\right) f^{\prime}\left(z_{2}\right)\right| \rightarrow 0,
$$

wenn $z_{1}$ und $z_{2}$ im Winkel gegen $z_{0}$ konvergieren und $(68,4)$ gilt, womit $(68,2)$ bewiesen ist. Andererseits aber liefert die Behauptung $(43,3)$ des Satzes XVI, auf $h(z)$ angewandt, da ja jetzt $k_{+}\left(\vartheta_{0}\right)=k_{-}\left(\vartheta_{0}\right)=\Delta\left(\vartheta_{0}\right)=0$ ist, die Behauptung $(68,3)$ unmittelbar.

Wir fassen unsere Resultate zusammen im

Satz XXIII. Ist unter den Voraussetzungen des Satzes $I V \gamma=0$ und besitzen die in $z_{0}$ bzw. wo zusammenstossenden Randzweige von $G_{1}$ bzw. G in diesen Punkten $L$-Tangenten, so gilt, wenn das Punktepaar $z_{1}, z_{2}$ aus dem Innern von $G_{1}$ so gegen $z_{0}$ konvergiert, dass dabei $\left|\frac{z_{2}-z_{0}}{z_{1}-z_{0}}\right|$ zwischen festen positiven Schranken bleibt,

$$
\arg f^{\prime}(z)+\arg \left(z-z_{0}\right) \rightarrow c,
$$

für eine Konstante $c$, wenn $z$ allseitig gegen $z_{0}$ geht;

$(68,6)$

$$
\frac{f^{\prime}\left(z_{2}\right)}{f^{\prime \prime}\left(z_{1}\right)} \sim\left(\frac{z_{1}-z_{0}}{z_{2}-z_{0}}\right)^{\frac{\pi}{\gamma_{1}}},
$$

wenn $z_{1}$ und $z_{2}$ im Winkel gegen $z_{0}$ gehen;

$$
\frac{\lg \left|f^{\prime}(z)\right|}{\lg \left|z-z_{0}\right|} \rightarrow-\frac{\gamma_{1}}{\pi}
$$

wenn $z$ aus $G_{1}$ im Winkel gegen $z_{0}$ geht. 